\title{
Cordyceps spp.: A Review on Its Immune-Stimulatory and Other Biological Potentials
}

Gitishree Das ${ }^{1}$, Han-Seung Shin ${ }^{2}$, Gerardo Leyva-Gómez ${ }^{3}$, Maria L. Del Prado-Audelo ${ }^{3}$, Hernán Cortes ${ }^{4}$, Yengkhom Disco Singh ${ }^{5}$, Manasa Kumar Panda ${ }^{6}$, Abhay Prakash Mishra ${ }^{7}$, Manisha Nigam ${ }^{8}$, Sarla Saklani ${ }^{9}$, Praveen Kumar Chaturi ${ }^{8}$, Miquel Martorell ${ }^{10}$, Natália Cruz-Martins ${ }^{11,12,13}$, Vineet Sharma ${ }^{14}$, Neha Garg ${ }^{15}$, Rohit Sharma ${ }^{14}$ and Jayanta Kumar Patra ${ }^{1 *}$

\begin{abstract}
${ }^{1}$ Research Institute of Biotechnology and Medical Converged Science, Dongguk University-Seoul, Goyangsi, South Korea, ${ }^{2}$ Department of Food Science and Biotechnology, Dongguk University-Seoul, Goyangsi, South Korea, ${ }^{3}$ Departamento de Farmacia, Facultad de Química, Universidad Nacional Autónoma de México, Ciudad de México, Mexico, ${ }^{4}$ Laboratorio de Medicina Genómica, Departamento de Genética, Instituto Nacional de Rehabilitación Luis Guillermo Ibarra Ibarra, Ciudad de México, Mexico, ${ }^{5}$ Department of Post-Harvest Technology, College of Horticulture and Forestry, Central Agricultural University, Pasighat, India, ${ }^{6}$ Environment and Sustainability Department, CSIR-Institute of Minerals and Materials Technology, Bhubaneswar, India, ${ }^{7}$ Adarsh Vijendra Institute of Pharmaceutical Sciences, Shobhit University, Saharanpur, India, ${ }^{8}$ Department of Biochemistry, H. N. B. Garhwal University, Srinagar Garhwal, India, ${ }^{9}$ Department of Pharmaceutical Chemistry, H. N. B. Garhwal University, Srinagar Garhwal, India, ${ }^{10}$ Department of Nutrition and Dietetics, Faculty of Pharmacy, and Centre for Healthy Living, University of Concepción, Concepción, Chile, ${ }^{11}$ Faculty of Medicine, Alameda Prof. Hernani Monteiro, University of Porto, Porto, Portugal, ${ }^{12}$ Institute for Research and Innovation in Health, University of Porto, Porto, Portugal, ${ }^{13}$ Laboratory of Neuropsychophysiology, Faculty of Psychology and Education Sciences, University of Porto, Porto, Portugal, ${ }^{14}$ Department of Rasa Shastra and Bhaishajya Kalpana, Faculty of Ayurveda, Institute of Medical Sciences, Banaras Hindu University, Varanasi, India, ${ }^{15}$ Department of Medicinal Chemistry, Faculty of Ayurveda, Institute of Medical Sciences, Banaras Hindu University, Varanasi, India
\end{abstract}

Young-Ji Shiao,

National Research Institute of Chinese Medicine, Taiwan Ulrike Lindequist, University of Greifswald, Germany

*Correspondence: Jayanta Kumar Patra jkpatra.cet@gmail.com

Specialty section: This article was submitted to

Ethnopharmacology,

a section of the journal

Frontiers in Pharmacology

Received: 03 September 2020 Accepted: 02 December 2020 Published: 08 February 2021

Citation:

Das G, Shin H-S, Leyva-Gómez G, Prado-Audelo MLD, Cortes $\mathrm{H}$, Singh YD, Panda MK, Mishra AP, Nigam M, Saklani S, Chaturi PK,

Martorell M, Cruz-Martins N,

Sharma V, Garg N, Sharma R and Patra JK (2021) Cordyceps spp.: A Review on Its Immune-Stimulatory and

Other Biological Potentials.

Front. Pharmacol. 11:602364. doi: 10.3389/fphar.2020.602364
In recent decades, interest in the Cordyceps genus has amplified due to its immunostimulatory potential. Cordyceps species, its extracts, and bioactive constituents have been related with cytokine production such as interleukin (IL)-1 $\beta$, IL2, IL-6, IL-8, IL-10, IL-12, and tumor necrosis factor (TNF)- $\alpha$, phagocytosis stimulation of immune cells, nitric oxide production by increasing inducible nitric oxide synthase activity, and stimulation of inflammatory response via mitogen-activated protein kinase pathway. Other pharmacological activities like antioxidant, anti-cancer, antihyperlipidemic, antidiabetic, anti-fatigue, anti-aging, hypocholesterolemic, hypotensive, vasorelaxation, anti-depressant, aphrodisiac, and kidney protection, has been reported in pre-clinical studies. These biological activities are correlated with the bioactive compounds present in Cordyceps including nucleosides, sterols, flavonoids, cyclic peptides, phenolic, bioxanthracenes, polyketides, and alkaloids, being the cyclic peptides compounds the most studied. An organized review of the existing literature was executed by surveying several databanks like PubMed, Scopus, etc. using keywords like Cordyceps, cordycepin, immune system, immunostimulation, immunomodulatory, pharmacology, anti-cancer, anti-viral, clinical trials, ethnomedicine, pharmacology, phytochemical analysis, and different species names. This review collects and analyzes state-of-the-art about the properties of Cordyceps species along with ethnopharmacological properties, application in food, chemical compounds, extraction of bioactive compounds, and various pharmacological properties with a special focus on the stimulatory properties of immunity. 
Keywords: ethnopharmacology, cordyceps, cordycepin, natural medicine, immune system, immunostimulation, immunomodulatory, clinical studies

\section{INTRODUCTION}

The fungus Cordyceps spp. belongs to Tibetan medicine and consumers describe it as an important source of energy. Cordyceps spp. belongs to Ascomycota, Pyrenomycetes, Hypocreales, and Clavicepitaceae, and at least 700 species are known. The word Cordyceps originates from the Greek term "kordyle", which means "club", and the Latin etymon "ceps", which means "head" (Olatunji et al., 2018). Cordyceps species invade insects, arthropods, other fungi, and evades the host immune system by harmonizing the life cycle of its host with the intention of survival and multiplication. Their interaction with the host will produce different secondary metabolites (Olatunji et al., 2018), such as cordycepin, adenosine, guanosine, cordymin, $\gamma$-aminobutyric acid (GABA), exopolysaccharides, cordysinin A-E, among others (Liu Y et al., 2015).

The different species of Cordyceps have beneficial properties such as anti-cancer, anti-proliferative, anti-angiogenic, antimetastasis, apoptosis induction, anti-inflammatory, antioxidant, anti-fibrotic, anti-arteriosclerosis, anti-hypertensive, antithrombotic, antimalarial, antifungal, hypolipidemic, antidiabetic, hypoglycemic, anti-asthmatic, steroidogenesis, spermatogenic, anti-aging, and immunomodulatory effects (Liu Y et al., 2015). These properties are concentration-dependent, and in most cases, no adverse effects were reported, although the evaluation of isolated compounds such as cordycepin is preferred.

Interestingly, Cordyceps spp. contains different compounds with the ability to strengthen the response of the immune system and also to control its exacerbated response. Most of the information on the effect of Cordyceps on the immune system derives from studies in cancer. In particular, Cordyceps spp. increases the production of interleukin (IL)$1 \beta$, IL-2, IL-6, IL-8, IL-10, IL-12, and tumor necrosis factor (TNF)- $\alpha$, induces phagocytosis of macrophages, mononuclear cells, nitric oxide (NO) release, and stimulates the inflammatory response via the mitogen-activated protein kinase (MAPK) route (Lee et al., 2006; Wang $M$ et al., 2012). Furthermore, it presents synergism with interferon (INF) $-\gamma$ in the production of cytokines. These properties are attractive in the search for new applications where the stimulation in the immune system response is wanted. Therefore, this review collects and analyzes the state-of-theart about properties of Cordyceps spp. focused on the stimulatory properties of immunity.

\section{METHODOLOGY}

An organized review of the existing literature was executed by surveying pertinent peer-reviewed research articles, review articles, etc. from several available bibliographic databanks such as PubMed, SpringerLink, Elsevier journal, Science
Direct, Scopus databases, Google search, etc., using keywords and its combination like Cordyceps, cordycepin, natural medicine, immune system, immunostimulation, immunomodulatory, pharmacology, anti-cancer, anti-viral, clinical trials, ethnomedicine, pharmacology, phytochemical analysis, and different species names. Usually, the search was carried out in "title, abstract, and keyword" fields. In each search, normally the review articles were omitted, however, in some instances, some important review articles were also considered. Further only articles published in the English language were considered. Articles that were published with only basic ethnobotanical assessment reports which lack substantial proof of the claim were not included in the study.

\section{HABITAT, DISTRIBUTION, AND CHARACTERISTICS OF CORDYCEPS SPP.}

From the more than 700 species of mushrooms recognized on the genus Cordyceps, around 20 species parasitize on the genus Elaphomyces, meanwhile the remaining species do on insects and arthropods belonging to Arachnida, Hymenoptera, Isoptera, Coleoptera, Hemiptera, and Lepidoptera classes. This diversity of species includes the $C$. sinensis (Ophiocordyceps sinensis (Berk.) G.H.Sung, J.M.Sung, Hywel-Jones and Spatafora), C. ophioglossoides (Tolypocladium ophioglossoides (Ehrh.) Quandt, Kepler \& Spatafora), C. militaris (L.) Fr., C. gracilis (Grev.) Durieu \& Mont., C. sobolifera (Hill ex Watson), C. subsessilis Petch, C. gunnii (Berk.) Berk., C. cicadae S.Z. Shing, C. tuberculate (Lebert) Maire, C. scarabaeicola Kobayasi, C. minuta Kobayasi, C. myrmecophila Ces., C. canadensis Ellis \& Everh., C. nutans Pat., C. agriota A. Kawam., C. ishikariensis M. Zang, D. Liu and R. Hu, C. sphecocephala (Berk.) Sacc, C. konnoana Kobayasi \& Shimizu, C. nigrella Kobayasi \& Shimizu, C. pruinosa Petch, C. tricentri Yasuda, among others (Tuli et al., 2013a; Lo et al., 2013; Baral et al., 2015; Pal and Misra, 2018).

These species exhibit different characteristics such as pharmaceutical properties, making them attractive to traditional Chinese medicine (TMC) since the nineties, and being $C$. sinesis the most studied and applied. Their geographic distribution is mainly based on the host distribution; however, they can grow in high mountains at an altitude of 3,600-4,000 $\mathrm{m}$ above the sea level. Thus, Cordyceps spp. has been found in North America, Europe, and Asia, mostly in countries such as China, Japan, Nepal, Bhutan, Vietnam, Korea, and Thailand. In India, it is principally present in subalpine regions such as Kumaun Himalaya and Garhwal Himalaya (at higher altitudes) (Maity, 2013; Chakraborty et al., 2014). Furthermore, it has been reported that species such as C. gunnii (Berk.) Berk. was found in Australia (Olatunji et al., 2018). The composition of their metabolite makes them able to tolerate characteristic severe conditions at 
high altitudes (low temperature, lack of oxygen, and exposure to UV radiation).

On the other hand, the dispersion of this rare and interesting medicinal mushroom is carried out through air, rain, and insects; in its whole life cycle in three phases namely infection, parasitism, and saprophytism (Pal and Misra, 2018). In the first phase, Cordyceps spp. infects the host in the larval stage through ascospores, (released in the air from mature fruiting bodies during summer and early autumn), and germinate. In some cases, the infection is produced by the ingestion of food contaminated by Cordyceps spp. mycelia. The parasitic stage occurs after the infection, and during this phase, the Cordyceps spp. nurtures from the bowel of the host. The fungal cells spread throughout the body and proliferate rapidly during the winter, consuming all internal organs of the larva, leaving intact the exoskeleton. After that, the fungal cell transform into a white mass inside the larva's body (endosclerotium) (Tuli et al., 2013a; Baral et al., 2015). During this process, the environmental conditions are inclement, and the mushroom has to resist the snow and cold conditions. When the spring begins, and the outside temperature increases, the endosclerotium germinates and extrudes through the oral cavity of the host, maturating in summer, forming the fruiting body, and beginning to release ascospores (saprophytic stage). In this season, the fungus collection is carried out.

Traditionally, the primary collectors of these plants are the villagers, who collected it during the time of grazing practice (Baral et al., 2015). During months, the primary gatherers stay in the alpine regions to care for their pet animals (Yak) and collect the fungus and other medicinal plants (Panda, 2010). Local medicine men, who also visit the areas to collect the mushrooms, store the dried material to use it in the future. Due to the medicinal importance of Cordyceps spp., its popularity has increased besides the over-harvesting, triggering the scarcity of wild species. For this reason, since the 70s, many scientists have been searching for options to achieve the fermentation and cultivation of fungi isolated. Cordyceps spp. have been related to therapeutic properties and healing activities for several years; thus, they have been employed as treatment of different diseases in folk medicine.

\section{ETHNOPHARMACY AND TRADITIONAL USES OF CORDYCEPS SPP.}

For hundreds of years, Cordyceps has been utilized in traditional Chinese medicine (TCM) as a tonic to treat several conditions such as respiratory diseases, liver or renal problems, hyperglycemia, and cancer or tumor disorders. Similarly, Cordyceps spp. has been applied as an energy level and endurance enhancer, to improve erobic capacity, and to boost cellular immunity. It was officially classified as a drug in 1964 in Chinese Pharmacopoeia (Shashidhar et al., 2013), being C. sinensis and C. militaris (L.) Fr. the most frequent species employed.

In some regions such as China, Tibetan plateau, Bhutan, Nepal, and India, the dosage and the administration of $C$. sinensis are dependent on the knowledge and skills of local folk practitioners based on the use of a trial-and-error method (Maity, 2013). For example, some community dissolves the fungus in milk, and alcohol or hot water, to drink it as an enhancer of the desire and sexual potency and as a tonic for the mornings, respectively (Panda and Swain, 2011). The action of the mushroom merged with other bioactive molecules has been reported too. For instance, some folk healers prescribe the use of the Cordyceps spp. mixed with taxus leaf and root of ginseng as a cancer treatment.

Furthermore, C. sinensis has been described as nutritious food by the Chinese population, probably due to their composition presents nutritional components such as essential amino acids, vitamins (B1, B2, B12, and $\mathrm{K}$ ), and carbohydrates, among others. Remarkably, this fungus species is a dietary complement that complies with the U.S. Food and Drug Administration (FDA) considerations, which render the cordyceps a demand product in many countries (Wu et al., 2015).

On the other hand, Cordyceps spp. has been applied as a remedy for fatigue and weakness, slowing down the symptoms of altitude sickness and giving the patient a boost of energy. At advanced ages, people decrease aches and pains. Similarly, TCM specialists recommend the regular intake of $C$. sinensis to avoid infections, colds, and flues, due to its ability to decrease cough and phlegm, asthma as well as bronchial diseases (Lo et al., 2013). For these reasons, the Cordyceps spp. have been applied as a treatment for lung fibrosis, particularly in patients suffering from severe acute respiratory syndrome (SARS). Following the TCM beliefs, all these properties are related to the $C$. sinensis ability to enrich the lung yin and yang (Chiu et al., 2016a). The benefits of Cordyceps spp. have also been observed in athletes due to energy improvement derived from the increment of the cellular ATP level, which releases energy in muscle cells.

Similar to C. sinensis, the applications of C. militaris (L.) Fr. (found in China, Japan, Korea, and East Asia), are related to its properties as an energy enhancer, aphrodisiac source, and respiratory conditions treatment. Besides, hypoglycemic, antiinflammatory, antitumor, antibacterial, antifungal, antioxidant, and immuno-protective properties have been attributed to this species. Thus, it ranks second in the most commercialized species in China, Japan, and Korea, being considered a suitable cheaper substitute for C. sinensis (Chou et al., 2014).

Other species that have been utilized by the folk healers are $C$. pruinosa Petch, C. bassiana, C. cicadae S.Z. Shing, C. gunnii (Berk.) Berk., C. guangdongensis T.H. Li, Q.Y. Lin and B. Song, and C. ophioglossoides (T. ophioglossoides). The main applications of C. pruinosa Petch are in stomach diseases are inflammatory disorders. C. bassiana Z.Z. Li, C.R. Li, B. Huang and M.Z. Fan, has been used for skin conditions such as dermatitis and eczema. It also is applied as a biological insecticide for pest control (Wu et al., 2015; Olatunji et al., 2018). In TCM, C. cicadae S.Z. Shing has been used to treat infantile convulsions, elevation of temperature, and tremors. Moreover, therapeutic activities such as antitumor, immunoregulatory, and reno-protective have been attributed to this species (Olatunji et al., 2018). Similarly, C. gunnii (Berk.) Berk. exhibits immunomodulatory activity, an enhancer effect on the memory, and delay of 
TABLE 1 | Chemical compounds of Cordyceps spp. (mentioned here only those compounds which were tested in a laboratory) adapted from Olatunji et al. with permission (Originally some portion of Table 3) (Olatunji et al., 2018).

\begin{tabular}{|c|c|c|c|}
\hline Compounds & Species & Mode of action & References \\
\hline Palmitic acid & C. militaris (L.) Fr. & Inactive & Yoon et al. (2015) \\
\hline \multicolumn{4}{|l|}{ Linoleic acid } \\
\hline \multicolumn{4}{|l|}{ Linoleic acid methyl ester } \\
\hline Cordytropolone & Cordyceps spp. BCC 1681 & $\begin{array}{l}\text { Antimalarial, cytotoxicity against } \mathrm{KB}, \mathrm{BC}-1 \text {, and } \\
\text { Vero cells lines }\end{array}$ & $\begin{array}{l}\text { Seephonkai et al. } \\
\text { (2001) }\end{array}$ \\
\hline Helvolic acid & C. taii Z.Q. Liang and A.Y. Liu & $\begin{array}{l}\text { Cytotoxicity against HeLa, HepG2, 95-D and } \\
\text { SW1990 cell lines }\end{array}$ & Dou et al. (2013) \\
\hline Cordycepiamide A & C. ninchukispora C.H. Su and H.H. Wang) G.H. & Anti-inflammatory & Reis et al. (2013) \\
\hline Cordycepiamide B & Sung, J.M. Sung, Hywel-Jones and Spatafora & & \\
\hline \multicolumn{4}{|l|}{ Cordycepiamide C } \\
\hline \multicolumn{4}{|l|}{ Cordycepiamide D } \\
\hline \multicolumn{4}{|l|}{$\begin{array}{l}\text { N-(2-hydroxybenzyl)acetamide } \\
\text { (-)-Syringaresinol }\end{array}$} \\
\hline Cordycerebroside A & C. militaris (L.) Fr. & Anti-inflammatory & Chiu et al. (2016b) \\
\hline \multicolumn{4}{|l|}{ Soyacerebroside I } \\
\hline \multicolumn{4}{|l|}{ Glucocerebroside } \\
\hline Ergosterol peroxide & C. cicadae S.Z. Shing & Anti-inflammatory, renoprotective & Kuo et al. (2003) \\
\hline Ergosta-4,6,8 (14),22-tetraen-3-one & C. sinensis (Berk.) Sacc. & Antitumor & Bok et al. (1999) \\
\hline Jiangxienone & $\begin{array}{l}\text { C. jiangxiensis Z.Q. Liang, A.Y. Liu \& Yong C. } \\
\text { Jiang }\end{array}$ & Cytotoxicity against SGC-7901 and A549 cell lines & Zhan et al. (2005) \\
\hline $\mathrm{H} 1-\mathrm{A}$ & C. sinensis (Berk.) Sacc. & Renoprotective & Yang et al. (2003) \\
\hline Cordyheptapeptide A & Cordyceps spp. BCC 16173 & $\begin{array}{l}\text { Cytotoxicity against } \mathrm{KB}, \mathrm{BC}, \mathrm{NCl}-\mathrm{H} 187 \text {, and Vero } \\
\text { cells lines }\end{array}$ & $\begin{array}{l}\text { Isaka et al. } \\
\text { (2007b) }\end{array}$ \\
\hline Cordyheptapeptide B & Cordyceps spp. BCC 16176 & $\begin{array}{l}\text { Cytotoxicity against } \mathrm{KB}, \mathrm{BC}, \mathrm{NCl}-\mathrm{H} 187 \text {, and Vero } \\
\text { cells lines }\end{array}$ & \\
\hline Cordycommunin & $\begin{array}{l}\text { Ophiocordyceps Communis Hywel-Jones and } \\
\text { Samson }\end{array}$ & Anti-mycobacterial & $\begin{array}{l}\text { Haritakun et al. } \\
\text { (2010) }\end{array}$ \\
\hline Beauvericin J & C. cicadae S.Z. Shing & Cytotoxicity against HepG2 and HepG2/ADM cell & Wang et al. (2004) \\
\hline Beauvericin & & lines & \\
\hline \multicolumn{4}{|l|}{ Beauvericin A } \\
\hline \multicolumn{4}{|l|}{ Beauvericin B } \\
\hline \multicolumn{4}{|l|}{ Beauvericin $\mathrm{E}$} \\
\hline Cordyceamide A & C. sinensis (Berk.) Sacc. & Cytotoxicity against L929, A375, and Hela cell lines & Jia et al. (2009) \\
\hline \multicolumn{4}{|l|}{ Cordyceamide B } \\
\hline Cycloaspeptide A & & Cytotoxicity against HeLa and MCF-7 cell lines & Zhang et al. \\
\hline Cycloaspeptide C & & & (2009) \\
\hline \multicolumn{4}{|l|}{ Cycloaspeptide F } \\
\hline \multicolumn{4}{|l|}{ Cycloaspeptide G } \\
\hline Cordycepin & & $\begin{array}{l}\text { Neuroprotection, anti-metastatic, anti-platelet ag- } \\
\text { gregation, anti-inflammatory activity, anti-cancer }\end{array}$ & $\begin{array}{l}\text { Yu H M et al. } \\
(2006)\end{array}$ \\
\hline $\mathrm{N}^{6}$-hydroxyethyl-adenosine & C. pruinosa Petch & Anti-inflammatory, $\mathrm{Ca}^{2+}$ antagonistic & Meng et al. (2015) \\
\hline Guanosine & C. sinensis (Berk.) Sacc. & Immunomodulatory & Yu L et al. (2006) \\
\hline CordysininB & & Anti-inflammatory & Yang et al. (2011) \\
\hline Dimethylguanosine & & Antioxidant and HIV-1 protease & Jiang et al. (2011) \\
\hline Ergosterol & & Anti-inflammatory, anti-fibrotic & $\begin{array}{l}\text { Nallathamby et al. } \\
\text { (2015) }\end{array}$ \\
\hline Ergosteryl-3-O- $\beta$-D-glucopyranoside & & Anti-inflammatory, antioxidant & Bok et al. (1999) \\
\hline $\begin{array}{l}\text { 5a,8a-epidioxy-22E-ergosta-6,9-(11)-22- } \\
\text { trien-33ß-ol }\end{array}$ & & Cytotoxic against HL-60 cell line & $\begin{array}{l}\text { Matsuda et al. } \\
\text { (2009) }\end{array}$ \\
\hline \multicolumn{4}{|l|}{$5 \alpha, 6 \alpha$-epoxy-5a-ergosta-7,22-dien-3 $\beta$-ol } \\
\hline 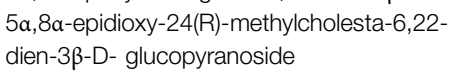 & & Antitumor & Bok et al. (1999) \\
\hline Cardinalisamide A & C. cardinalis G.H. Sung \& Spatafora & Antitrypanosomal & Umeyama et al. \\
\hline Cardinalisamide B & & & (2014) \\
\hline \multicolumn{4}{|l|}{ Cardinalisamide C } \\
\hline Cicadapeptin I & C. heteropoda Kobayasi & Antibacterial and antifungal & Krasnoff et al. \\
\hline Cicadapeptin II & & & $(2005)$ \\
\hline Cordycepsidone A & C. dipterigena Berk. and Broome & Antifungal & Varughese et al. \\
\hline Cordycepsidone B & & & $(2012)$ \\
\hline Cyclo (L-Pro-L-Val) & C. sinensis (Berk.) Sacc. & Antioxidant, anti-inflammatory & Yang et al. (2011) \\
\hline \multicolumn{4}{|l|}{ Cyclo (L-Phe-L-Pro) } \\
\hline \multicolumn{4}{|l|}{ Cyclo (L-Pro-L-Tyr) } \\
\hline Cordycepoid A & C. bifusispora O.E. Erikss. & Inactive & Lu et al. (2013) \\
\hline
\end{tabular}


TABLE 1 | (Continued) Chemical compounds of Cordyceps spp. (mentioned here only those compounds which were tested in a laboratory) adapted from Olatunji et al. with permission (Originally some portion of Table 3) (Olatunji et al., 2018).

\begin{tabular}{|c|c|c|c|}
\hline Compounds & Species & Mode of action & References \\
\hline $\begin{array}{l}\text { Cordysinin A } \\
\text { Flazin } \\
\text { Perlolyrine }\end{array}$ & C. sinensis (Berk.) Sacc. & Anti-inflammatory, antioxidant & Yang et al. (2011) \\
\hline Cordyformamide & C. brunnearubra BCC 1395 & Antimalarial & 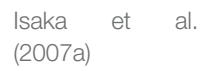 \\
\hline Deacetylcytochalasin C & C. taii Z.Q. Liang and A.Y. Liu & $\begin{array}{l}\text { Cytotoxicity against } 95-\mathrm{D}, \mathrm{A}-549 \text {, and } \mathrm{HL}-7702 \text { cell } \\
\text { lines }\end{array}$ & Li et al. (2015) \\
\hline Zygosporin D & & $\begin{array}{l}\text { Cytotoxicity against } 95-\mathrm{D}, \mathrm{A}-549 \text {, and } \mathrm{HL}-7702 \text { cell } \\
\text { lines }\end{array}$ & \\
\hline $\begin{array}{l}\text { Cordypyridone A } \\
\text { Cordypyridone B } \\
\text { Cordypyridone C } \\
\text { Cordypyridone D } \\
\text { 1-Dehydroxycordypyridone A }\end{array}$ & C. nipponica Kobayasi & Antimalarial & Isaka et al. (2001) \\
\hline $\begin{array}{l}3^{\prime}, 4^{\prime}, 7 \text {-Trihydroxyisoflavone } \\
\text { Diadzein }\end{array}$ & C. sinensis (Berk.) Sacc. & $\begin{array}{l}\text { Antioxidant } \\
\text { Antioxidant, anti-inflammatory }\end{array}$ & Yang et al. (2011) \\
\hline $\begin{array}{l}\text { Rugulosin } \\
\text { Skyrin }\end{array}$ & C. formosana Kobayasi \& Shimizu & Cytotoxicity against $\mathrm{CHO}$ cell line & Lu et al. (2014) \\
\hline 6'-O-desmethylES-242-4 & Cordyceps spp. BCC 16173 & Antimalarial & $\begin{array}{l}\text { Isaka et al. } \\
\text { (2007b) }\end{array}$ \\
\hline $\begin{array}{l}\text { Annullatin A } \\
\text { Annullatin B } \\
\text { Annullatin C } \\
\text { Annullatin D } \\
\text { Annullatin E }\end{array}$ & C. annullata Kobayasi \& Shimizu & Cannabinoid receptors agonist & Asai et al. (2012) \\
\hline $\begin{array}{l}\text { Erythrostominone } \\
\text { Deoxyerythrostominone } \\
\text { 4-O-methyl-erythrostominone } \\
\text { Epierythrostominol } \\
\text { Deoxyerythrostominol }\end{array}$ & C. unilateralis (Tul.) Sacc. & $\begin{array}{l}\text { Antimalarial, cytotoxicity against } \mathrm{BC}, \mathrm{KB} \text {, and Vero } \\
\text { cell lines }\end{array}$ & $\begin{array}{l}\text { Kittakoop et al. } \\
\text { (1999) }\end{array}$ \\
\hline $\begin{array}{l}\text { Cordycepol } \\
\text { Cordycepol B } \\
\text { Cordycepol C } \\
\text { Cordycol }\end{array}$ & $\begin{array}{l}\text { C. ophioglossoides (T. ophioglossoides) (Ehrh.) } \\
\text { link }\end{array}$ & $\begin{array}{l}\text { Cytotoxicity against HeLa and HepG2 cell lines } \\
\text { Inactive } \\
\text { Cytotoxicity against HeLa and HepG2 cell lines }\end{array}$ & Sun et al. (2013) \\
\hline Ophicordin & C. sinensis (Berk.) Sacc. & Antifungal & $\begin{array}{l}\text { Kneifel et al. } \\
(1977)\end{array}$ \\
\hline $\begin{array}{l}\text { Terreusinone A } \\
\text { Pinophilin C } \\
\text { CryptosporioptideA }\end{array}$ & C. gracilioides Kobayasi & Protein tyrosine phosphatases inhibitor & Wei et al. (2015) \\
\hline $\begin{array}{l}\text { Furancarboxylicacid } \\
\text { Hydroxy-2-methyl-4-pyrone }\end{array}$ & C. sinensis (Berk.) Sacc. & Anti-inflammatory, antioxidant & Yang et al. (2011) \\
\hline $\begin{array}{l}\text { Bassiamide A (KTH-7-1) } \\
\text { Bassiamate (KTH-7-2) } \\
\text { IPr-PEPhenol (KTH-13) } \\
\text { KTH-15-2 } \\
\text { KTH-17 }\end{array}$ & $\begin{array}{l}\text { C. bassiana Z.Z. Li, C.R. Li, B. Huang and } \\
\text { M.Z. Fan }\end{array}$ & Antiproliferative against C6 glioma cell & $\underset{(2015)}{\text { Kim J H et al. }}$ \\
\hline $\begin{array}{l}\text { 4-Quinolinol } \\
\text { 1-Naphthol }\end{array}$ & & Anti-inflammatory & $\underset{(2014)}{\text { Kim }}$ T W et al. \\
\hline
\end{tabular}

senescence (Zhu et al., 2012b; Zhu Z-Y et al., 2014). C. guangdongensis is employed against fatigue, avian influenza, inflammation, renal failure, and oxidation (Yan et al., 2013). On the other hand, C. ophioglossoides (T. ophioglossoides) has been used as food, presenting antitumor, estrogenic, and anti-aging products, besides its application in births to avoid excessive bleeding in women (Kawagishi et al., 2004; Olatunji et al., 2018).

The traditional consumption of Cordyceps spp. has been through an herbal product, and its massive marketing dates back to the beginning of the year 2000. In several countries, it is consumed as a food supplement due to its different health attributes. To date, it is a highly sought-after product since its fame increased accompanied by scientific evidence. Prices range up to $\$ 20,000$ per kilogram for wild $C$. sinensis, making it the most expensive mushroom in the world.

\section{CHEMICAL COMPOUNDS OF CORDYCEPS SPP.}

The genus Cordyceps spp. contains a large number of chemical compounds and their derivatives in the form of secondary 


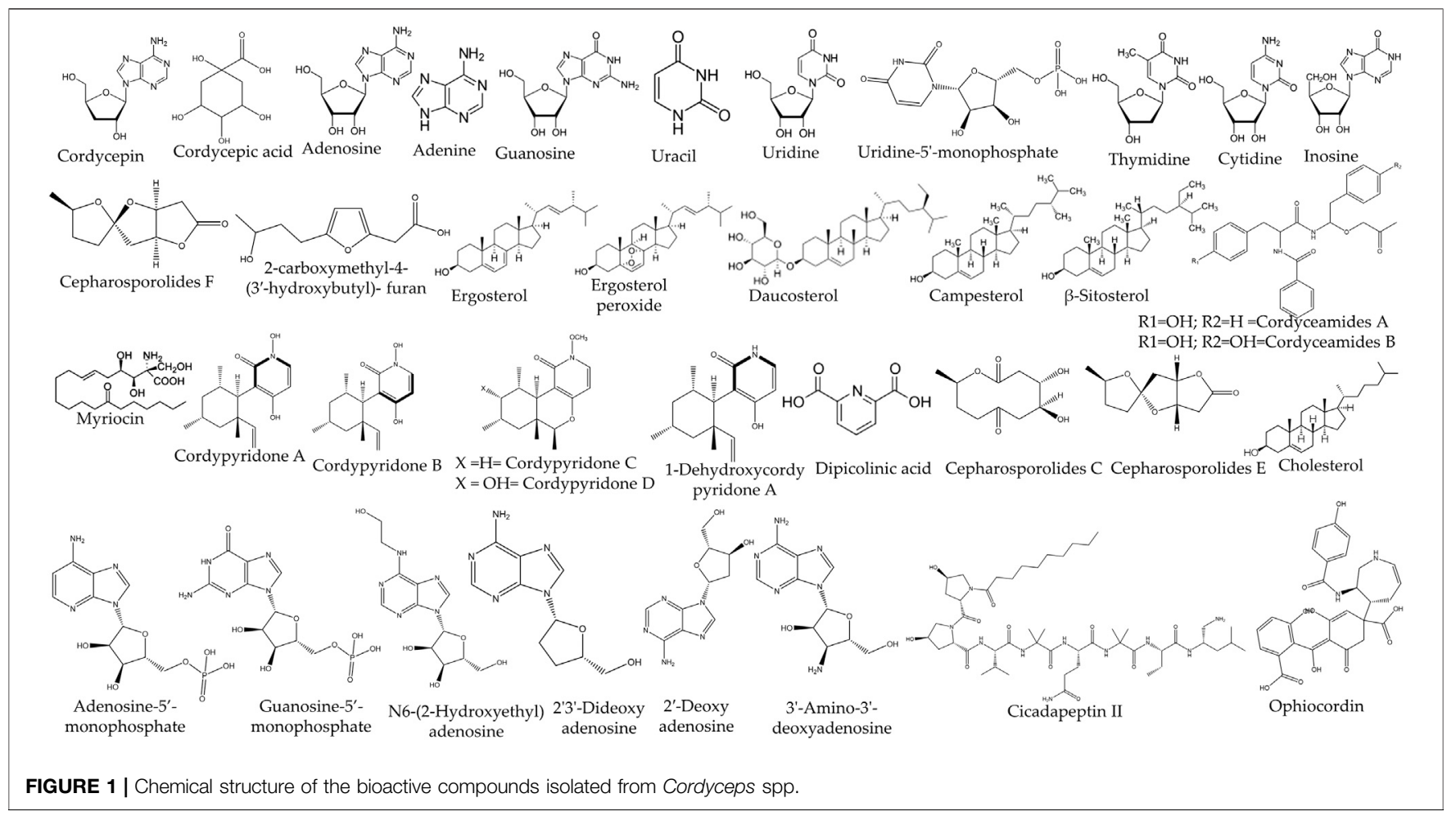

metabolites. The presence of such diverse chemical compounds makes them quite intriguing in analyzing therapeutic effects and pharmacological studies. Major chemical compounds such as nucleosides, sterols flavonoids, cyclic peptides, phenolic, bioxanthracenes, polyketides, and alkaloids are found in Cordyceps species (Table 1, Figure 1). While in most of the Cordyceps species, cyclic peptides are present in large quantity as compared to other molecules. Besides that, cordycepin and cordycepic acid (CA) are also prominently present in some species of Cordyceps spp. such as C. militaris (L.) Fr.. The presence of cordycepin (3'-deoxyadenosine) and $2^{\prime}$ deoxyadenosine in $C$. sinensis was characterized by using atomic attractive reverberation (NMR) and infrared spectroscopy (IR) (Shunzhi and Jingzhi, 1996). In addition to this, a class of saccharides and polysaccharides such as cyclofurans, a cyclic ring of five-carbon sugars, heteropolysaccharides beta-glucans, beta-mannans crossconnected beta-mannan polymers, and complex polysaccharides comprising of both five and six carbon sugars were also discovered from Cordyceps spp. Even though, Cordyceps spp. contains a lot of bioactive molecules, it also has immunosuppressive compounds, cyclosporine usually found in Cordyceps subsessilis Petch (Segelken, 1996). Besides this, some immunosuppressant compounds were also isolated from the closely related Cordyceps species Isaria sinclairii (Berk.) Lloyd (Mizuno, 1999).

\section{Cordycepin and Cordycepic Acid}

Cordycepin and CA are prominently found in C. militaris (L.) Fr. They are important bioactive molecules having potential therapeutic applications (Huang et al., 2003). Structurally, cordycepin is $3^{\prime}$ deoxyadenosine and CA is D-mannitol. Cordycepin is an analog of adenosine derivatives which themselves differentiated from adenosine nucleoside by the absence of one oxygen molecule at third position carbon of ribose sugar. Different types of extraction methods of this compound can be followed, however, one of the most frequently used methods in which acetonitrile and water mixed in the ratio of $5: 95 \mathrm{v} / \mathrm{v}$ at a flow rate of $1.0 \mathrm{ml} / \mathrm{min}$ can be adapted (Ikeda et al., 2008). Cordycepin has been associated with various therapeutic ailments including intracellular targets, nucleic acid, apoptosis, and cell cycle. This diverse role of cordycepins in cellular molecular activities is due to its resemblance to adenosine (Tuli et al., 2013b). On the other hand, CA is structurally an isomer of quinic acid possessing various potential medicinal applications. Previously, CA structure was concluded as 1,3,4,5tetrahydroxycyclohexane-1-carboxylic acid (Chatterjee et al,, 1957) later, it was found to be crystalline substance of D-mannitol (Sprecher and Sprinson, 1963). It differs mainly from quinic acid as it forms dextrorotatory instead of forming lactone (Chatterjee et al., 1957). There is a great variation of CA content in the Cordyceps spp. However, in C. sinensis, it is usually 7-29\% with differing in growing stages of the Cordyceps spp. (Jiang, 1987). CA plays a great influence in treating liver fibrosis (Guo and Friedman, 2007), diuretic, plasma osmotic pressure, and anti-free radical properties (Nomani et al., 2014).

\section{Polysaccharides}

Cordyceps spp. contains different types of polysaccharide components. The fruiting bodies of Cordyceps spp. consist of 3-8\% polysaccharides ( $\mathrm{Li}$ et al., 2001a). It was known that the 
polysaccharides obtained from Cordyceps species are medicinally important and can play as one of the main constituents in drug formulation (Ukai et al., 1983; Wasser, 2002). These polysaccharides can effectively control the blood sugar level in the body (Kiho et al., 1993), show antimetastatic and antitumor effects (Nakamura et al., 1999), and also have anti-influenza, immunoprotective, and antioxidant effects. Cordyceps spp. polysaccharides represent structurally diverse biologically active macromolecules of wide physiochemical properties. These polysaccharides are either intracellular or extracellular. Molecular weight greater than 16,000 is shown to have effective antitumor properties (Zhou et al., 2009). The polysaccharides derived from edible, medicinal mushrooms were successfully shown to exhibit antitumor and immunomodulating properties which were firstly reported from the fruit body of Lentinusedodes in 1969 (Chihara et al., 1969). Therefore, a large number of edible and medicinal polysaccharides including Cordyceps spp. have been rigorously investigated over the past 30 years. Apart from this, many novel antitumor and immunomodulatory polysaccharides have been developed and commercialized (Wasser, 2002; Xiao et al., 2002; Xiao et al., 2003). The important species of Cordyceps spp. from which polysaccharides have been isolated and developed which possess antitumor activities includes C. sinensis, C. cicadae S.Z. Shing, C. ophioglossioides (Tolypocladium ophioglossoides (Ehrh.) Quandt, Kepler \& Spatafora), C. militaris (L.) Fr. and C. kyushuensis A. Kawam. As per the study, the polysaccharides combined with other chemotherapeutic drugs showed synergism and increased body-resistance (Xiao et al., 2002; Yang et al., 2005; Zhang W et al., 2005; Chen et al., 2006). Polysaccharides derived from Cordyceps spp. primarily include glucan, mannan, heteroglycan, and glycoprotein but only $\beta$ - $(1 \rightarrow 3)$ glucan, galactosaminoglycan, and proteopolysaccharide from $C$. cicadae S.Z. Shing, C. ophioglossioides and Cordyceps spp. showed antitumor activity (Xiao et al., 2002; Xiao et al., 2003).

\section{Proteins and Nitrogenous Compounds}

Cordyceps spp. contains all essential amino acids, proteins, peptides, polyamines. Additionally, the Cordyceps spp. contains several rare cyclic dipeptides, including cyclo-[Gly-Pro], cyclo[Leu-Pro], cyclo-[Val-Pro], cyclo-[Ala-Leu], and cyclo-[ThrLeu]. Significant quantities of polyamines were also detected, such as 1,3-diamino propane, cadaverine, spermidine, spermine, and putrescine (Mizuno, 1999; Mishra and Upadhyay, 2011). Other nitrogenous compound like putrescine and putrescine, ware also identified (Mizuno, 1999).

\section{Nucleotides/Nucleotide Derivatives}

Besides the other components, Cordyceps spp. is rich in nucleotide and its derivatives. In $C$. sinensis, nucleosides are the main component contributing to therapeutic applications (Li et al., 2001b). Nucleosides such as adenine, adenosine, inosine, cytidine, cytosine, guanine, uridine, thymidine, uracil, hypoxanthine, and guanosine have been isolated from $C$. sinensis. Among the nucleotide components, guanosine has the highest content ratio than other components (Shaoping et al., 2001). There is a usual difference between the nature of nucleosides from that of normal and cultured C. sinensis (Li et al., 2001c). Many specific nucleosides that are not found elsewhere in nature can be found in the Cordyceps spp. which includes several distinct deoxyuridin structures, adenosine, $2^{\prime}-3^{\prime}$ dideoxyadenosine, hydroxyethyladenosine, cordycepin triphosphate, guanidine, and deoxyguanidine. Adenosine and cordycepin ( $3^{\prime}$-deoxyadenosine) possess multiple functions such as immunomodulatory, antioxidant, etc., Chen and Chu (Chen and Chu, 1996), identified cordycepin by using magnetic resonance (NMR) and infrared spectroscopy (IR) in a C. sinensis sample. In identification of cordycepin, several analytical methods and techniques including RP-HPLC (Shiao et al., 1994; Yu H M et al., 2006; Yu L et al., 2006), HPLC-ESI-MS (Huang L F et al., 2004), and HPLC-DAD (Jiang et al., 2008) were adopted.

\section{Sterols and Fatty Acid}

Fungi contain sterols in the form of ergosterol an essential part of the great therapeutic important part of vitamin $\mathrm{D}_{2}$. Cordyceps spp. has identified a host of several sterol-type compounds and a few of these names: ergosterol, ergosterol-3, ergosterol peroxide, 3-sitosterol, daucosterol, and campesterol (Zhou et al., 2009). In Cordyceps spp., the existence of ergosterol varies depending on their growth stage, i.e., ergosterol was $1.44 \mathrm{mg} / \mathrm{g}$ in Cordyceps spp. mycelium, while $10.68 \mathrm{mg} / \mathrm{g}$ in fruit bodies (Li et al., 2011). Some derivatives of Cordyceps spp. are found in D-3-ergosterol, 3sitosterol, daucosterol, and campesterol, and so on. It is important to mention that HPLC in C. sinensis detects ergosterol ( $\mathrm{Li}$ and $\mathrm{Li}$, 1991; Li et al., 2004).

The fatty acids found in Cordyceps spp. can be classified loosely into two kinds of fatty acids, saturated and unsaturated. Cordyceps spp. are more common and can compensate for up to $57.84 \%$ of unsaturated fatty acids (Zhou et al., 2009). Fatty acid such as lauric acid, myrtic acid, pentadecanic acid, palmitic acid, linoleic acid, oleic acid, stearic acid, and docosanic acid, are reported in Cordyceps spp. (Mishra and Upadhyay, 2011). Zhu et al. (1998), reported that 28 saturated and unsaturated fatty acids and their derivatives were isolated from $C$. sinensis along with polar compounds include several alcohols and aldehydes. The unsaturated fatty acids have various physiological activities, including decreased lipid blood and cardiovascular disease. Two methanol isolated sterols displayed antitumor sequence, and were detected by $1 \mathrm{D}$ and 2D NMR spectroscopy in their structure (Bok et al., 1999). Usage of pressurized fluid extraction (PLE), derivation of trimethyl silyl (TMS), GC-MS, cholesterol, campesterol, and $\beta$-sitosterol, like ergosterol from natural (wild) C. sinensis were described (Yang et al., 2009).

\section{Other Constituents}

In addition to the core ingredients, $C$. sinensis is made mostly from proteins, peptides, polyamine, both important amino acids, and other unusual cyclic dipeptides such as cyclo-[Gly-Pro-], cyclo-[Leu-Pro-], cyclo-[Val-Pro] and cyclo-[Thr-Leu]. Cyclic dipeptides including cyclo-(Leu-Pro) and cyclo-(Phe-Pro) were seen to have antimicrobial activity and anti-mutagenic properties in the battle against the production of vancomycin-resistant 
Enterococcus (VRE) and pathogenic yeasts (Rhee, 2004). As per the study, cyclic (bacterial) dipeptides inhibit the development of aflatoxin (Yan et al., 2004) and protein rates differ greatly in the sum of dead larvae (29.1\%), fruit body (30.4\%), and mycelial fermentation $(14.8 \%)$. The major amino acids are present in the larvae such as glutamic acid, aspartic acid, and amino acid (Hsu et al., 2002). The anti-inflammatory and antinociceptive properties of cordymine, a peptide isolated from $C$. sinensis medicinal mushrooms, have been reported (Qian et al., 2012).

The exopolysaccharide fraction (EPSF), is derived from the harvested $C$. sinensis supernatant. The cultured supernatant has been collected and then processed with the three times in volume of $95 \%$ ethanol for precipitation. As a consequence, a large amount of EPSFs was found on the soil (Zhang et al., 2008). EPSF has a wide spectrum of pharmacologic effects, with immunomodulatory and antitumor effects are most important (Sheng et al., 2011). EPSF has already shown that it can scavenge free radicals, promote the differentiation of cell cancer, and improve the ability of antitumor activity by triggering many immune responses (Sheng et al., 2011). Ion-exchanging and size chromatography is used to isolate polysaccharide (PS) from cultivated C. sinensis mycelia. Polysaccharide fraction (PSF) has been extracted from $C$. sinensis fungus has a relaxing effect on macrophage (Chen $W$ et al., 2010). PSF has been shown to transform $M_{2}$ macrophages to $M_{1}$ phenotypes by activating the nuclear factor kappa-B (NF- $\mathrm{B})$ pathway. PSF also has immunomodulatory impacts, including many other polysaccharides (Chen et al., 2012). In a study to document the effect of $C$. sinensis on T-lymphocyte subsets of chronic renal failure patients, it was reported that different components of Cordyceps spp. polysaccharides enhanced the cellular immune function, phagocytic function of monocytemacrophage, improved renal functions, spleen, and thymus index (Guan et al., 1992).

\section{EXTRACTION AND ISOLATION OF MAJOR COMPOUNDS FROM CORDYCEPS SPP.}

\section{Extraction}

A few extraction strategies have been used for solvents extraction utilized for the confinement of particular bio-dynamic mixes (Chen P X et al., 2013). Different extracts exhibit significant biological activities.

\section{Aqueous Extraction}

In aqueous extraction, water is used as an extraction medium due to the polar nature of the molecule and extracts polar compounds like-nucleosides and polysaccharides. Sun et al. (2003) standardized the suitable conditions for aqueous extraction as water: plant powder ratio (2.5:1), $\mathrm{pH}-7.5-8.0$, and $24 \mathrm{~h}$ extraction time (Sun et al., 2003). Moreover, in hot water extraction, the yield varies between $25-30 \%$ with potential health benefits like antioxidant activities (Yamaguchi et al., 2000a; Gu et al., 2003).

\section{Alcoholic Extraction}

An alcoholic extraction method mainly methanol, ethanol, aqueous methanol, and aqueous ethanol are used for extraction as per bioactive principles. Yamaguchi et al. studied the alcoholic extraction because it allows a higher extraction of bioactive molecules, such as nucleosides, polysaccharides, proteins, as a result, exhibits strong antioxidant activity and preserves B-cell function and provides protection (Yamaguchi et al., 2000a; Kan et al., 2012). Another study revealed that methanol extract obtained from $C$. sinensis was found to have cytotoxicity impact on cancer cell lines (Jia et al., 2009).

\section{Ethyl Acetate Extraction}

Ethyl acetic acid derivation concentrate $C$. sinensis includes an intensification range not as similar to water and alcohol. Although the yield in this technique is small, the technique includes sugar, adenosine, ergosterol, and cordycepin, which are differentiated by ergosterol and similar mixes as a significant class of dynamic portion. The cause of apoptosis in human pre-myelocytic leukemia HL60 is due to 2 days of treatment in ED $50 \pm 25 \mu \mathrm{g} / \mathrm{ml}$, as a result, restrains the proliferation of malignancy growth of the cell lines (Zhang et al., 2004; Wu et al., 2007). Further research is utilized to comprehend basic highlights and adequacy of dynamic mixes in ethyl acetic acid derivation extricate. Ethyl acetate extract of $C$. sinensis showed antioxidant and immunomodulatory potential (Wu et al., 2006; Wu et al., 2007).

\section{Supercritical Carbon Dioxide $\left(\mathrm{CO}_{2}\right)$ Extraction}

The extraction of supercritical $\mathrm{CO}_{2}$ has been an emerging technique in the chemical and food sectors in recent years. It is the best method carried out under moderate conditions and its purest form to extract bioactive compounds (especially non-polar compounds), without toxic organic solvents for extraction. Many literatures on simple and supercritical methods for the extraction of fluids in different fields are available (Pereira and Meireles, 2010). Ethanolic C. sinensis extract was fractionated with supercritical $\mathrm{CO}_{2}$ as an elution solvent, demonstrating its strong scavenging potential and inhibiting colorectal and hepatocellular cell development via the apoptosis cycle (Wang et al., 2005).

\section{PHARMACOLOGICAL POTENTIAL OF CORDYCEPS SPP.}

Plethora of naturally occurring chemical entities attributes to the broad and remarkable pharmacological activities of Cordyceps spp. (Zhu et al., 1998; Tuli et al., 2013a). Out of the diverse variety of species, $C$. sinensis is the most investigated one, as far as research and the inspection of its pharmacological potential is concerned (Paterson, 2008; Olatunji et al., 2018). Besides, other species includes C. militaris (L.) Fr.; C. pruinosa Petch; C. ophioglossoides (T. ophioglossoides); C. bassiana Z.Z. Li, C.R. Li, B. Huang and M.Z. Fan; C. guangdongensis T.H. Li, Q.Y. Lin and B. Song; C. gunnii (Berk.) Berk.; C. jiangxiensis Z.Q. Liang, A.Y. Liu \& Yong C. Jiang; C. kyushuensis A. Kawam.; C. 


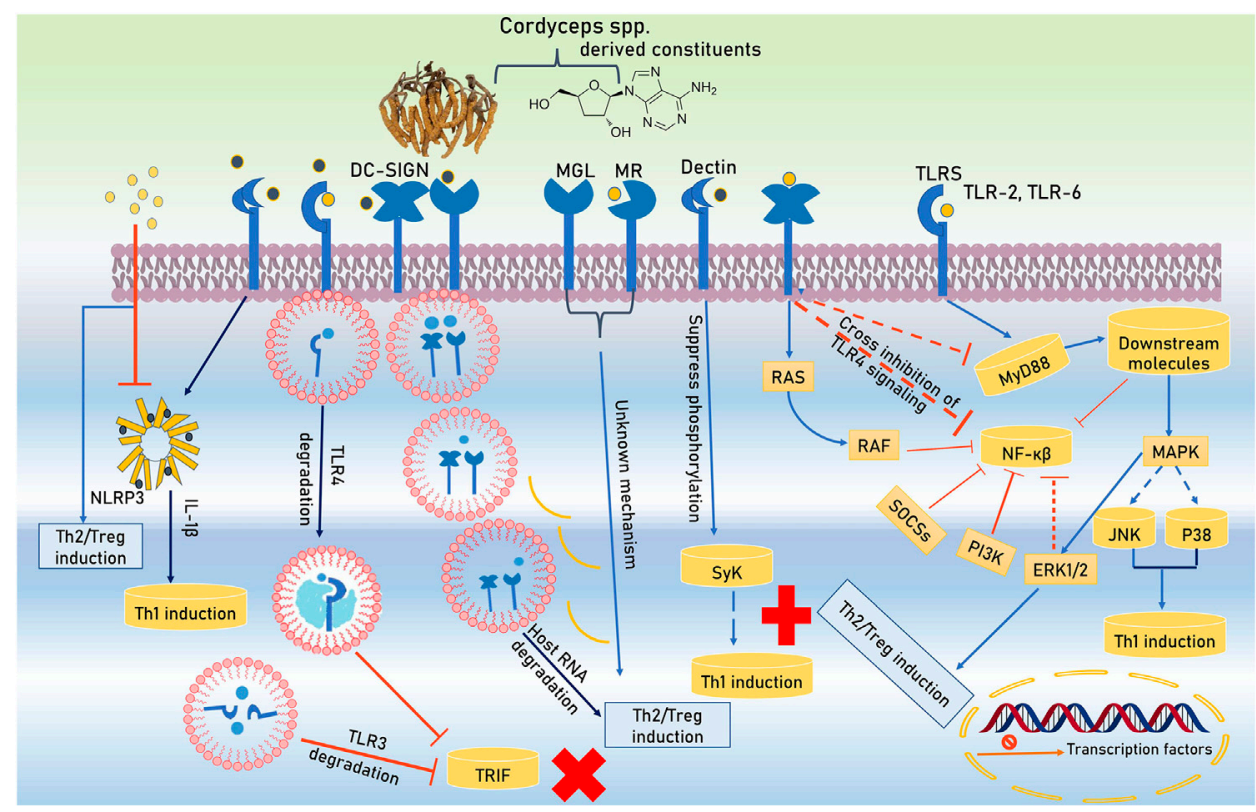

FIGURE 2 | Signal pathway activation by TLRs and CLRs and its interaction with the Cordyceps spp. derived constitutes.

pseudomilitaris Hywel-Jones and Sivichai; C. sphecocephala (Berk.) Sacc; C. soblifera (Hill ex Watson) and C. taii Z.Q. Liang and A.Y. Liu. The proposed applications of Cordyceps spp. in medicine include as immune-stimulatory, immunomodulatory, anti-inflammatory, antioxidant, antitumor, antimetastatic, antibacterial, antifungal, antimalarial, HIV-1 protease inhibitor, antihyperlipidemic, antiobesity, anti-diabetic, anti-arteriosclerosis, anti-thrombotic, anticoagulant, anti-fatigue (Qian et al., 2012; Liu Y et al., 2015). Details of the Cordyceps spp. induced pharmacological actions have been described as below.

\section{Immuno-Modulatory Action of Cordyceps spp.}

The immunomodulators are the substances or compounds that helps to control the immune system of the body. There are a number of compounds present in the Cordyceps spp. that possesses the immunomodulatory activity. Some of these are discussed below. Active constitutes of Cordyceps spp. are spotted by Toll-like receptors (TLRs) and C-type lectin receptors (CLRs) during initiation of immunomodulation and hyporesponsiveness in antigen-presenting cells (APCs). These active constituents not only alter the TLRs and CLRs expression in APCs but also masterfully manipulate their intracellular signaling. TLRs use the Toll/IL-1 receptor (TIR)-domain covering adapter proteins such as MyD88 and TRIF (TIR domain-containing adapter inducing IFN- $\beta$ ). Active bio-constituents of Cordyceps spp. ( $C$. cicadae S.Z. Shing, C. militaris (L.) Fr., C. sinensis, C. sobolifera (Hill ex Watson)) transmit TLR4 signaling to MAPK pathway and extracellular signal-related kinase one and 2 (ERK1/2) activation backing Treg/Th2 induction. Furthermore, coherence of DC-SIGN (dendritic cell-specific intercellular adhesion molecule-3-grabbing non-integrin) along with TLR4 enables active constituents of Cordyceps spp. to trigger unknown intracellular pathways that cross-inhibit MyD88 and NF- $\mathrm{BB}$ activation. These constituents are further restrained NF- $\kappa \mathrm{B}$ activity via the upregulation of negative regulators of TLRs signaling like a suppressor of cytokine signaling (SOCS) and phosphatidylinsoitol-3-kinase (PI3K) along with DC-SIGNmediated rapidly accelerated fibrosarcoma (RAF) signaling. In the prevention of priming Th1 cells, the role of NF- $\kappa \mathrm{B}$ is a core factor due to its support's inflammation by inhibition. The multiplicity of signaling pathways is improved by co-receptors' involvement of CLRs (DC-SIGN). Activated mannose receptor (MR) and macrophage galactose-type C-type lectin (MGL) helps for the differentiation of Treg/Th2. Degrading host key intracellular molecules is another strategy that Cordyceps spp. exploit to reprogram host immunity. Polysaccharide constituents of Cordyceps spp. degrades endosomal TLR2, TLR3, TLR4, TLR6, and host mRNA which provides Treg/Th2 responses support. The active bio-constituents stimulate Treg/Th2 cell priming which have been stated by CLRs involvements. NLRP3 inflammasome (NLRP3 and caspase-1) modulate inflammatory processes via secretion of IL-1 $\beta$ and Th1 intensification (Figure 2).

Xu et al. (1992) delineated the effects of the C. sinensis (ethanolic extract) on murine and human natural killer (NK) activity and on colony formation of B16 melanoma in mouse lungs, where they reported the augmentation of the in vivo and in vitro $\mathrm{NK}$ activities of the mouse. Moreover, the pre-incubation of peripheral blood mononuclear cells (PBMCs) with C. sinensis elevated in vitro NK activity of human PBMCs, whereas the colony formation of B16 melanoma in mouse lungs was reduced drastically. This report hinted at the $C$. sinensis 
TABLE 2 | Immunostimulatory and related bioactivities of Cordyceps spp.: in vitro and in vivo.

\begin{tabular}{lcccc}
\hline Activity & Species & In vitro/in \\
vivo & Refults
\end{tabular}

Immunostimulant

C. militaris (L.) Fr.

C. japonica Lloyd

C. bassiana Z.Z. Li, C.R. Li,

B. Huang and M.Z. Fan Z.Z.

Li, C.R. Li, B. Huang and Butanol fraction

M.Z. Fan

Methanol

C. gunnii (Berk.) Berk.

Polysaccharide

C. pruinosa Petch

Polysaccharide

C. taii Z.Q. Liang and A.Y. Liu

Polysaccharides

C. sinensis (Berk.) Sacc.
Aqueous and methanol

Forced swimming performances, immobilizing stress

LPS-activated macrophages

Molecular basis of inhibition of cytokine expression HMNC proliferation

Macrophage phagocytosis along with humoral and cellular immunity

Spleen lymphocytes proliferation, peritoneal macrophage (PMphi) phagocytosis, and CTL

Splenic $T$ cell and Mphi phagocytosis

Renal injury in endotoxemic rats

Splenic $T$ cell and Mphi
phagocytosis

Adenosine
guanosine
Polysaccharide

Exopolysaccharide

Aqueous

Chloroquine and bafilomycin A

Methanol and RAW264.7 cells

Macrophages proliferation phagocytosis B16 melanoma-bearing mice

Lupus-prone (NZB/NZW) F1 hybrids

Macrophage $\mathrm{J} 774$ cell

Raw264.7 macrophage cell

Bone marrow-derived dendritic cells (BM-DCs)

Lymphoproliferative response, natural killer cell (NK) $\downarrow N O$, ROS, TNF- $\alpha$ production, Lee and Hong NF-kB activation and MAPKs (2011)

pathways, melanoma growth

$\downarrow N O, \quad T N F-\alpha$ and $I L-1 \beta$ Lee et al. production (2010)

$\downarrow N O$, TNF- $\alpha$ and activates mac- Lee et al. rophages through the MAPKs (2015) and NF- $\mathrm{kB}$ signaling pathways

$\uparrow$ Liver enzyme activities, \lipid Shin et al. peroxidation

$\downarrow$ Expression of IL-12, IFN- $\gamma \quad$ Byeon et al. (2011a)

\IL-12 and TNF- $\alpha$ expression, Byeon et al. Syk, JAK-2, and ERK (2011b)

$\downarrow$ HMNC proliferation, $E_{50}=$ Weng et al. $32.5 \pm 5.2 \mu \mathrm{g} / \mathrm{ml}$, $\uparrow \mathrm{IL}-2$ and (2002) IFN- $\gamma$

$\uparrow$ Thymus and spleen indexes, Zhu et al macrophage phagocytosis, the (2012b) proliferation of splenic cells, level of IFN- $\gamma$ and TNF- $\alpha$, $\downarrow$ IL-4 $\downarrow$ Spleen lymphocytes prolifera- Xiao et al tion, PMphi phagocytosis of (2004b) neutral red and CTL

$\uparrow$ Proliferation of activated Liu and Fei splenic T cell and cellular im- (2001) mune functions

\Oxidative stress and inflamma- (Chiu et al., tory cytokines, NF- $\kappa$ B activation, 2014) †body's cellular antioxidant defense system.

†Proliferation of activated (Liu and Fei, splenic T cell and cellular im- 2001) mune functions

$\downarrow N O, \uparrow I L-1 \beta, T N F-\alpha \quad(Y u$ et al. 2007)

Activation of the MAPK and NF- (Cheong et al., $\kappa B$ signaling pathways 2016)

$\uparrow$ Neutral red uptake capacity, Zhang Q et al. spleen lymphocyte proliferation, (2005) $\downarrow$ levels of $\mathrm{Bcl}-2$

$\uparrow$ Survival, CD8 ${ }^{+} T$ cells $\%, \downarrow$ pro- $\quad$ (Chen et al., teinuria, titers of anti-double- 2009) stranded DNA antibody CD4 ${ }^{+}$

$\mathrm{T}$ cells\%

†Phagocytosis (Jia and Lau, 1997)

Stimulate the release of (Wang et al. cytokines 2011)

Activation of BM-DCs in a TLR9- (Xiao et al., dependent manner 2010)

Inhibited blastogenesis re- (Kuo et al., sponse, NK cell activity, IL-2, 1996) and TNF- $\alpha$ production

(Continued on following page) 
TABLE 2 | (Continued) Immunostimulatory and related bioactivities of Cordyceps spp.: in vitro and in vivo.

\begin{tabular}{|c|c|c|c|c|c|}
\hline Activity & Species & Extract/Compound & $\begin{array}{l}\text { In vitro/in } \\
\text { vivo }\end{array}$ & Results & References \\
\hline \multirow[t]{2}{*}{$\begin{array}{l}\text { Immunomodulatory and } \\
\text { antioxidant }\end{array}$} & C. militaris (L.) Fr. & Polysaccharides & $\begin{array}{l}\text { Cyclophosphamide-induced } \\
\text { immunosuppression }\end{array}$ & $\begin{array}{l}\uparrow \text { Spleen lymphocyte activity, } \\
\text { macrophage function, SOD, } \\
\text { catalase, GPx, and TAOC level } \\
\text { and the spleen and thymus in- } \\
\text { dices, } \downarrow \text { MDA level }\end{array}$ & $\begin{array}{l}\text { Wang } M \text { et al. } \\
(2012)\end{array}$ \\
\hline & & Polysaccharides & $\begin{array}{l}\text { Viscera index, leukocyte count, } \\
\text { differential leukocyte count, lgG } \\
\text { levels }\end{array}$ & $\begin{array}{l}\text { Upregulated the expression of } \\
\text { TNF- } \boldsymbol{\alpha}, \mathrm{IFN}-\boldsymbol{\gamma} \text {, and IL-1 } \boldsymbol{\beta} \text { mRNA, } \\
\uparrow \text { spleen and thymus indices, the } \\
\text { spleen lymphocyte activity, the } \\
\text { total quantity of white blood } \\
\text { cells, and IgG function, } \downarrow \mathrm{MDA}\end{array}$ & $\begin{array}{l}(\text { Liu et al., } \\
2016)\end{array}$ \\
\hline Immunosuppressive & C. gunnii (Berk.) Berk. & Polysaccharide & Cytotoxic T Iymphocytes & $\begin{array}{l}\text { Inhibiting cellular immunologic } \\
\text { and humoral immunologic } \\
\text { function }\end{array}$ & $\begin{array}{l}\text { (Xiao et al., } \\
2004 a)\end{array}$ \\
\hline \multirow[t]{13}{*}{ Anti-inflammatory } & $\begin{array}{l}\text { C. bassiana Z.Z. Li, C.R. Li, } \\
\text { B. Huang and M.Z. Fan }\end{array}$ & 1,9-Dimethylguanine & $\begin{array}{l}\text { Reporter gene assay and } \\
\text { mRNA analysis }\end{array}$ & $\begin{array}{l}\text { Blockade of luciferase activity } \\
\text { caused by NF-кB and AP-1, } \\
\text { suppress the mRNA levels of } \\
\text { COX- } 2 \text { and TNF- } \boldsymbol{\alpha}\end{array}$ & $\begin{array}{l}\text { (Suh et al., } \\
2017 \text { ) }\end{array}$ \\
\hline & & Butanol fraction & $\begin{array}{l}\text { LPS-induced inflammation in } \\
\text { RAW } 264.7 \text { cells }\end{array}$ & $\begin{array}{l}\text { \NO, iNOS, COX-2, } \quad \text { IкB, } \\
\text { MAPKs activation, JNK, and } \\
\text { p38 phosphorylation }\end{array}$ & $\begin{array}{l}\text { (Yoon et al., } \\
2017)\end{array}$ \\
\hline & & $\begin{array}{l}\text { 4-Isopropyl-2,6-bis(1- } \\
\text { phenylethyl) phenol }\end{array}$ & $\begin{array}{l}\text { LPS and sodium nitroprusside } \\
\text { treated RAW264.7 cells }\end{array}$ & $\begin{array}{l}\downarrow N O \text { and ROS production, } \\
\text { mRNA expression, NF-кB } \\
\text { activation }\end{array}$ & $\begin{array}{l}\text { (Yang et al., } \\
2015 b)\end{array}$ \\
\hline & & Aqueous & LPS-treated RAW264.7 cells. & $\begin{array}{l}\downarrow \text { COX-2, IL-12, and iNOS, Syk } \\
\text { kinase activity } \uparrow I L-10\end{array}$ & $\begin{array}{l}\text { (Yang et al., } \\
2017 \text { ) }\end{array}$ \\
\hline & & Butanol fraction & & $\begin{array}{l}\text { \NO and ROS production, and } \\
\text { |кB/NF-кB pathway, JNK, and } \\
\text { p38 activation }\end{array}$ & $\begin{array}{l}\text { Kim T W et al. } \\
(2014)\end{array}$ \\
\hline & C. cicadae S.Z. Shing & Ergosterol peroxide & Human T cells & $\begin{array}{l}\downarrow T \text {-cell proliferation, } \| \mathrm{L}-2, \quad \mathrm{IL}-4 \text {, } \\
\| \mathrm{L}-10 \text {, and } \mathrm{IFN}-\boldsymbol{\gamma}, \mathrm{AP}-1 \text { proteins } \\
\text { expression }\end{array}$ & $\begin{array}{l}\text { (Kuo et al., } \\
2003)\end{array}$ \\
\hline & & $\begin{array}{l}\mathrm{N}^{6}-(2-H y d r o x y e t h y l) \\
\text { adenosine }\end{array}$ & LPS-induced pro-inflammatory & $\begin{array}{l}\downarrow \text { TLR4-mediated NF-кB signal- } \\
\text { ing pathway }\end{array}$ & $\begin{array}{l}(\mathrm{Lu} \text { et al., } \\
2015)\end{array}$ \\
\hline & & & $\mathrm{CCl}_{4}$-induced liver fibrosis & $\begin{array}{l}\downarrow \text { ¿UN and SCr levels, IL-12 and } \\
\text { TNF- } \boldsymbol{\alpha} \text { expression, TGF- } \boldsymbol{\beta} 1 / \\
\text { CTGF }\end{array}$ & $\begin{array}{l}\text { (Kim et al., } \\
\text { 2018) }\end{array}$ \\
\hline & $\begin{array}{l}\text { C. guangdongensis T.H. Li, } \\
\text { Q.Y. Lin and B. Song }\end{array}$ & Aqueous & $\begin{array}{l}\text { Chronic bronchitis caused by } \\
\text { tobacco smoking }\end{array}$ & $\begin{array}{l}\downarrow \text { Bronchial lesions and inflam- } \\
\text { matory cell infiltration }\end{array}$ & $\begin{array}{l}\text { (Yan et al., } \\
\text { 2014) }\end{array}$ \\
\hline & C. militaris (L.) Fr. & Aqueous & $\begin{array}{l}\text { Dextran sodium sulfate- } \\
\text { induced acute colitis }\end{array}$ & $\begin{array}{l}\text { Attenuated body weight loss, } \\
\text { diarrhea, gross bleeding, } \\
\text { lepithelial damage, loss of gob- } \\
\text { let cells, loss of crypts, infiltration } \\
\text { of inflammatory cells }\end{array}$ & $\begin{array}{l}\text { (Han et al., } \\
2011)\end{array}$ \\
\hline & & $\begin{array}{l}\text { Cordycerebroside A } \\
\text { soyacerebroside I and } \\
\text { glucocerebroside }\end{array}$ & RAW264.7 macrophages & $\begin{array}{l}\downarrow \text { Accumulation of pro- } \\
\text { inflammatory iNOS and COX-2 } \\
\text { protein expression }\end{array}$ & $\begin{array}{l}\text { (Chiu et al., } \\
\text { 2016b) }\end{array}$ \\
\hline & C. pruinosa Petch & & RAW264.7 macrophage cells & $\begin{array}{l}\downarrow \text { NO production, TNF-a, ROS, } \\
\text { IL-6, iNOS, phosphorylation of } \\
\text { p65/p50 }\end{array}$ & $\begin{array}{l}\text { Kim H G et al. } \\
(2014)\end{array}$ \\
\hline & & Methanol & & $\begin{array}{l}\downarrow \mathbb{L}-1 \boldsymbol{\beta}, \text { TNF- } \boldsymbol{\alpha}, \quad \text { COX-2, iNOS, } \\
\text { NF- } \mathbf{k B} \text { activation }\end{array}$ & $\begin{array}{l}\text { (Kim et al., } \\
\text { 2003) }\end{array}$ \\
\hline $\begin{array}{l}\text { Anti-inflammatory and } \\
\text { anti-cancer }\end{array}$ & C. militaris (L.) Fr. & Cordycepin & $\begin{array}{l}\text { LPS/IFN- } \boldsymbol{\gamma} \text {-stimulated macro- } \\
\text { phages and colon 205, PC-3, } \\
\text { and HepG2 cells }\end{array}$ & $\begin{array}{l}\downarrow N O, T N F-\alpha \text { and } I L-12 \text { produc- } \\
\text { tion, } \quad I_{50}=7.5,6.3, \text { and } \\
7.6 \mu \mathrm{g} / \mathrm{ml}\end{array}$ & $\begin{array}{l}\text { (Rao et al., } \\
2010)\end{array}$ \\
\hline
\end{tabular}

(Continued on following page) 
TABLE 2 | (Continued) Immunostimulatory and related bioactivities of Cordyceps spp.: in vitro and in vivo.

\begin{tabular}{|c|c|c|c|c|c|}
\hline Activity & Species & Extract/Compound & $\begin{array}{l}\text { In vitro/in } \\
\text { vivo }\end{array}$ & Results & References \\
\hline \multirow[t]{14}{*}{ Antioxidant } & C. cicadae S.Z. Shing & $\begin{array}{l}\text { Water-soluble } \\
\text { polysaccharides }\end{array}$ & $\begin{array}{l}\text { Total reducing power and } \\
\text { scavenging activities }\end{array}$ & $\begin{array}{l}\mathrm{IC}_{50}=28.99 \mu \mathrm{g} / \mathrm{ml} \text { (DPPH scav- } \\
\text { enging), } 0.19 \text { and } 0.30 \mathrm{mg} / \mathrm{m} \\
\mathrm{L} \mathrm{mg} / \mathrm{ml} \text { (hydroxyl and superox- } \\
\text { ide anion radicals) }\end{array}$ & $\begin{array}{l}\text { (Song et al., } \\
2018 \text { ) }\end{array}$ \\
\hline & $\begin{array}{l}\text { C. formosana Kobayasi \& } \\
\text { Shimizu }\end{array}$ & Aqueous & DPPH and ROS scavenging & Strong antioxidant capability & $\begin{array}{l}\text { Wang Y W } \\
\text { et al. (2015) }\end{array}$ \\
\hline & C. gunnii (Berk.) Berk. & Polysaccharide & D-galactose-induced & $\downarrow$ LPO, GPx, $\uparrow S O D$, catalase & $\begin{array}{l}\text { (Zhu et al., } \\
\text { 2009) }\end{array}$ \\
\hline & & & & $\downarrow M D A, G P x, \uparrow S O D$ & $\begin{array}{l}\text { (Zhu et al., } \\
2011)\end{array}$ \\
\hline & C. japonica Lloyd & Aqueous and methanol & TBA reactant assay & $\begin{array}{l}\uparrow \text { Cytosolic SOD, catalase, and } \\
\text { GSH-px, } \downarrow \text { MDA }\end{array}$ & $\begin{array}{l}\text { (Shin et al., } \\
\text { 2001) }\end{array}$ \\
\hline & & Ethanol & DPPH & $\begin{array}{l}\mathrm{IC}_{50}=163 \mu \mathrm{g} / \mathrm{ml}, \quad \uparrow S O D, \\
\text { catalase }\end{array}$ & $\begin{array}{l}\text { (Jung et al., } \\
2009 \text { ) }\end{array}$ \\
\hline & $\begin{array}{l}\text { C. jiangxiensis Z.Q. Liang, } \\
\text { A.Y. Liu \& Yong C. Jiang }\end{array}$ & Polysaccharide & DPPH & $\mathrm{EC}_{50}=18.06 \mathrm{mg} / \mathrm{ml}$ & $\begin{array}{l}\text { (Xiao et al., } \\
2011)\end{array}$ \\
\hline & C. kyushuensis A. Kawam. & Methanol & $\begin{array}{l}\text { Scavenging effect on hydroxyl } \\
\text { radical }\end{array}$ & $\mathrm{IC}_{50}=1.5-4.8 \mathrm{mg} / \mathrm{ml}$ & $\begin{array}{l}\text { (Zhang et al., } \\
\text { 2015) }\end{array}$ \\
\hline & C. militaris (L.) Fr. & Polysaccharides & $\begin{array}{l}\text { DPPH, hydroxyl and superox- } \\
\text { ide radical }\end{array}$ & Strong antioxidant capability & $\begin{array}{l}\text { Chen } X \text { et al. } \\
(2013)\end{array}$ \\
\hline & & Methanol & & $\begin{array}{l}\downarrow \text { LPO also scavenge reducing } \\
\text { power and free radicals. }\end{array}$ & $\begin{array}{l}\text { (Reis et al., } \\
\text { 2013) }\end{array}$ \\
\hline & C. pruinosa Petch & Polysaccharide & $\begin{array}{l}\text { DPPH, hydroxyl, and superox- } \\
\text { ide radical }\end{array}$ & $\begin{array}{l}\downarrow \text { LPO also scavenge reducing } \\
\text { power and free radicals. }\end{array}$ & $\begin{array}{l}(\text { Lu et al., } \\
2016)\end{array}$ \\
\hline & C. sinensis (Berk.) Sacc. & Polysaccharide & $\begin{array}{l}\text { PC12 cells against hydrogen } \\
\text { peroxide-induced injury }\end{array}$ & $\uparrow S O D$, GSH, $\downarrow$ MDA & $\begin{array}{l}\text { (Gu et al., } \\
2003)\end{array}$ \\
\hline & & Aqueous and ethanol & $\begin{array}{l}\text { DPPH, hydroxyl, and superox- } \\
\text { ide radical }\end{array}$ & $\begin{array}{l}\downarrow L P O, \quad \text { scavenge reducing } \\
\text { power and free radicals }\end{array}$ & $\begin{array}{l}\text { (Yamaguchi } \\
\text { et al., 2000a) }\end{array}$ \\
\hline & & Exopolysaccharide & Trolox equivalent antioxidant & 35-40 $\mu \mathrm{mol}$ Trolox/g & $\begin{array}{l}\text { (Leung et al., } \\
\text { 2009) }\end{array}$ \\
\hline $\begin{array}{l}\text { Antioxidant } \\
\text { and } \\
\text { Immunoenhancing }\end{array}$ & C. taii Z.Q. Liang and A.Y. Liu & Polysaccharides & D-galactose-induced aging & $\begin{array}{l}\text { Superoxide anion-free radical } \\
\left(\mathrm{EC}_{50}=2.04-2.49 \mathrm{mg} / \mathrm{ml}\right), \uparrow \\
\text { SOD, catalase, GSH, } \uparrow \mathrm{MDA}\end{array}$ & $\begin{array}{l}\text { (Xiao et al., } \\
2012 b)\end{array}$ \\
\hline Free radical scavenging & $\begin{array}{l}\text { C. bassiana Z.Z. Li, C.R. Li, } \\
\text { B. Huang and M.Z. Fan }\end{array}$ & Methanol & DPPH radical inhibition & $\begin{array}{l}47.7 \% \text { scavenging activity of } \\
\text { stage } 3\end{array}$ & $\begin{array}{l}\text { (Hyun et al., } \\
\text { 2013) }\end{array}$ \\
\hline \multirow[t]{11}{*}{ Anti-tumor } & C. sinensis (Berk.) Sacc. & Methanol & $\begin{array}{l}\text { K562, Jurkat, WM-1341, HL- } \\
60 \text {, and RPMI-8226 cells }\end{array}$ & $\begin{array}{l}10-40 \% \text { at } 10 \mu \mathrm{g} / \mathrm{ml} \text { inhibitor to } \\
\text { the proliferation }\end{array}$ & $\begin{array}{l}\text { (Bok et al., } \\
1999)\end{array}$ \\
\hline & & Polysaccharide & U937 cells & $\begin{array}{l}78-83 \% \text { growth inhibition rate, } \\
\uparrow I F N-\boldsymbol{\gamma} \text {, and TNF- } \boldsymbol{\alpha}\end{array}$ & $\begin{array}{l}\text { (Chen et al., } \\
1997)\end{array}$ \\
\hline & & Cordycepin & $\begin{array}{l}\text { K562, Vero, Wish, Calu-1, and } \\
\text { Raji tumor cell lines }\end{array}$ & Significantly inhibited & $\begin{array}{l}\text { (Kuo et al., } \\
1994)\end{array}$ \\
\hline & C. japonica Lloyd & Ethanol & Sarcoma-180 tumor cells & $\begin{array}{l}\uparrow P h a g o c y t o s i s \text { and acid phos- } \\
\text { phatase activity }\end{array}$ & $\begin{array}{l}\text { (Shin et al., } \\
\text { 2003) }\end{array}$ \\
\hline & C. gunnii (Berk.) Berk. & Polysaccharide & S180 cells & Stronger inhibition at $800 \mu \mathrm{g} / \mathrm{ml}$ & $\begin{array}{l}\text { (Zhu et al., } \\
2016 \mathrm{a})\end{array}$ \\
\hline & & & K562 cell & $56.65 \%$ tumor inhibition ratio & $\begin{array}{l}\text { (Zhu et al., } \\
\text { 2012a) }\end{array}$ \\
\hline & & & H22 cell & $45.3 \%$ tumor inhibition ratio & $\begin{array}{l}\text { Zhu Z-Y et al. } \\
(2014)\end{array}$ \\
\hline & & & K562 cells & $69.92 \%$ tumor inhibition ratio & $\begin{array}{l}\text { (Zhu et al., } \\
2013)\end{array}$ \\
\hline & & $\begin{array}{l}\text { Selenium enriched } \\
\text { polysaccharide }\end{array}$ & SKOV-3 cells & $\begin{array}{l}\text { Stimulate apoptosis through } \\
\text { p53-Bax-caspase pathway }\end{array}$ & $\begin{array}{l}\text { (Sun et al., } \\
\text { 2018) }\end{array}$ \\
\hline & & Polysaccharide & S180 cell & $85 \%$ tumor inhibition ratio & $\begin{array}{l}\text { (Zhu et al., } \\
\text { 2016b) }\end{array}$ \\
\hline & C. cicadae S.Z. Shing & Ethanol & SGC-7901 cells & $\begin{array}{l}\downarrow \text { Proliferation of SGC-7901 } \\
\text { cells, } \uparrow \text { calpain- } 1 \text {, caspase-12, } \\
\text { and caspase- } 9 \text { expression }\end{array}$ & $\begin{array}{l}\text { (Xie et al., } \\
2019)\end{array}$ \\
\hline $\begin{array}{l}\text { Anti-tumor } \\
\text { and antimetastatic }\end{array}$ & C. taii Z.Q. Liang and A.Y. Liu & Chloroform & A549 and SGC-7901 cells & $\begin{array}{l}\mathrm{IC}_{50}=30.2 \text { and } 65.7 \mu \mathrm{g} / \\
\mathrm{ml}, \uparrow \mathrm{GPx}\end{array}$ & $\begin{array}{l}\text { Liu R M et al. } \\
(2015)\end{array}$ \\
\hline
\end{tabular}

(Continued on following page) 
TABLE 2 | (Continued) Immunostimulatory and related bioactivities of Cordyceps spp.: in vitro and in vivo.

\begin{tabular}{|c|c|c|c|c|c|}
\hline Activity & Species & Extract/Compound & $\begin{array}{l}\text { In vitro/in } \\
\text { vivo }\end{array}$ & Results & References \\
\hline \multirow[t]{7}{*}{ Anti-cancer } & C. cicadae S.Z. Shing & Aqueous & $\begin{array}{l}\text { MHCC97H human hepatocel- } \\
\text { lular carcinoma cells }\end{array}$ & $\begin{array}{l}\text { \MHCC97H cells growth via } \\
\text { G2/M cell cycle arrest }\end{array}$ & $\begin{array}{l}\text { Wang } \mathrm{H} \text { et al. } \\
(2014)\end{array}$ \\
\hline & $\begin{array}{l}\text { C. formosana Kobayasi \& } \\
\text { Shimizu }\end{array}$ & Aqueous & $\begin{array}{l}\text { A549 lung cancer, MDA-mb- } \\
231 \text { breast cancer, Huh7 liver } \\
\text { cancer, and HL-60 leukemia } \\
\text { cells }\end{array}$ & $\begin{array}{l}\mathrm{IC}_{50}=1.0 \mathrm{mg} / \mathrm{ml}(\mathrm{A} 549 \mathrm{cells}) \\
\mathrm{IC}_{50}=0.53 \mathrm{mg} / \mathrm{ml} \text { (MDA-mb- } \\
231 \text { cells), } \quad \mathrm{IC}_{50}=0.44 \mathrm{mg} / \mathrm{ml} \\
\text { (Huh7 cells), } I_{50}=0.19 \mathrm{mg} / \\
\mathrm{ml}(\mathrm{HL}-60 \text { cells), } \downarrow \text { breast tumor } \\
\text { size }\end{array}$ & $\begin{array}{l}\text { Wang } \mathrm{J} \text { et al. } \\
(2014)\end{array}$ \\
\hline & C. kyushuensis A. Kawam. & & U937 and K562 cells & $\begin{array}{l}\mathrm{IC}_{50}=31.23 \mu \mathrm{g} / \mathrm{ml} \text { and } \\
62.5 \mu \mathrm{g} / \mathrm{ml}\end{array}$ & $\begin{array}{l}\text { (Zhao et al., } \\
2018)\end{array}$ \\
\hline & $\begin{array}{l}\text { C. jiangxiensis Z.Q. Liang, } \\
\text { A.Y. Liu \& Yong C. Jiang }\end{array}$ & Chloroform & $\begin{array}{l}\text { Gastric adenocarcinoma cell } \\
\text { line SGC-7901 }\end{array}$ & $\begin{array}{l}\mathrm{IC}_{50}=10 \mu \mathrm{g} / \mathrm{ml}, \uparrow \text { caspase-3 } \\
\text { activity }\end{array}$ & $\begin{array}{l}\text { (Xiao et al., } \\
2006)\end{array}$ \\
\hline & C. militaris (L.) Fr. & Curdlan & Dendritic cell maturation & $\begin{array}{l}\uparrow \text { CD40, CD80, CD86, MHC-I, } \\
\text { MHC-II molecules, IL-12, IL-1 } \boldsymbol{\beta} \text {, } \\
\text { TNF- } \boldsymbol{\alpha} \text {, IFN- } \boldsymbol{\alpha} \boldsymbol{\beta} \text { expression, } \\
\text { phosphorylation of ERK, p38, } \\
\text { JNK, and NF- } \mathbf{k B}, \text { p50/p65 }\end{array}$ & $\begin{array}{l}\text { Kim H S et al. } \\
(2010)\end{array}$ \\
\hline & C. taii Z.Q. Liang and A.Y. Liu & Cytochalasin & 95-D, A-549 and HL-7702 cells & $\mathrm{IC}_{50}=3.67-4.04 \mu \mathrm{M}$ & (Li et al., 2015) \\
\hline & $\begin{array}{l}\text { C. sphecocephala (Klotzsch } \\
\text { ex Berk.) Berk. and M.A. } \\
\text { Curtis }\end{array}$ & Polysaccharides & HepG2, SKN-SH cells & $\begin{array}{l}\text { Activation of caspase- } 3 \text {, and } \\
\text { modulation of } \mathrm{Bcl}-2 \text { and } \mathrm{Bax}\end{array}$ & $\begin{array}{l}\text { (Oh et al., } \\
2008)\end{array}$ \\
\hline \multirow[t]{7}{*}{ Cytotoxicity } & C. bifusispora O.E. Erikss. & Methanol & $\mathrm{CHO}$ cells & $\begin{array}{l}\text { Cell death ratio }(4.14 \pm 0.25) \text { at } \\
4,000 \mu \mathrm{g} / \mathrm{ml} \quad \text { concentration, } \\
\mathrm{LD}_{50}>8.0 \mathrm{~g} / \mathrm{kg}\end{array}$ & $\begin{array}{l}(\text { Lu et al., } \\
2013)\end{array}$ \\
\hline & C. cicadae Shing. & $\begin{array}{l}\text { Beauvericin, beauvericin } \\
\mathrm{A} \text {, beauvericin } \mathrm{E} \text {, and } \\
\text { beauvericin } \mathrm{J}\end{array}$ & HepG2 and HepG2/ADM cells & $\begin{array}{l}\mathrm{IC}_{50}=2.40 \pm 0.37 \text { to } 14.48 \pm \\
1.68 \mu \mathrm{M}\end{array}$ & $\begin{array}{l}\text { Wang } J \text { et al. } \\
(2014)\end{array}$ \\
\hline & $\begin{array}{l}\text { C. formosana Kobayasi \& } \\
\text { Shimizu }\end{array}$ & Rugulosin and skyrin & $\mathrm{CHO}$ cells & $\begin{array}{l}\text { Rugulosin and skyrin } L_{50}= \\
18.3 \pm 0.2 \text { and } 103.7 \pm \\
5.9 \mu \mathrm{g} / \mathrm{ml}\end{array}$ & $\begin{array}{l}(\text { Lu et al., } \\
2014)\end{array}$ \\
\hline & $\begin{array}{l}\text { C. jiangxiensis Z.Q. Liang, } \\
\text { A.Y. Liu \& Yong C. Jiang }\end{array}$ & Jiangxienone & SGC-7901 cell and A549 cell & $\mathrm{IC}_{50}=1.38-2.93 \mu \mathrm{M}$ & $\begin{array}{l}\text { (Xiao et al., } \\
\text { 2012a) }\end{array}$ \\
\hline & & & HGC-27 & $\begin{array}{l}\text { DNA damage response } \\
\text { pathway }\end{array}$ & $\begin{array}{l}(\text { Lü et al., } \\
2014)\end{array}$ \\
\hline & C. pruinosa Petch & Cordycepol C, cordycol & HeLa and HepG2 & $\mathrm{IC}_{50}=12-33 \mu \mathrm{g} / \mathrm{ml}$ & $\begin{array}{l}\text { (Sun et al., } \\
2013)\end{array}$ \\
\hline & & Butanol fraction & HeLa & $\begin{array}{l}\text { Caspase-3- and -9-dependent } \\
\text { apoptosis, } \uparrow \text { proteolytic cleavage } \\
\text { of PARP and release of cyto- } \\
\text { chrome c, } \downarrow \text { Bcl-2/Bax protein } \\
\text { ratio }\end{array}$ & $\begin{array}{l}\text { Kim H G et al. } \\
(2010)\end{array}$ \\
\hline \multirow[t]{3}{*}{ Anti-diabetic } & C. cicadae S.Z. Shing & Crude polysaccharide & Alloxan-induced diabetic & $\begin{array}{l}\text { \Blood glucose, TC, TG, LDL, } \\
\text { MDA, urea, CREA, ALT, AST, } \\
\text { and ALP. } \uparrow \text { body weights, HDL, } \\
\text { SOD, GSH }\end{array}$ & $\begin{array}{l}\text { (Zhang et al., } \\
\text { 2018) }\end{array}$ \\
\hline & C. japonica Lloyd & Methanol & STZ-induced diabetes & $\begin{array}{l}\downarrow \text { serum glucose, glucose toler- } \\
\text { ance up to } 3 \mathrm{~h}\end{array}$ & $\begin{array}{l}\text { (Shim et al., } \\
\text { 2000) }\end{array}$ \\
\hline & C. militaris (L.) Fr. & Cordycepin & Alloxan-induced & $\begin{array}{l}\downarrow \text { Blood glucose, TC, TG, LDL, } \\
\text { MDA, urea, CREA, ALT, AST, } \\
\text { and ALP. } \text { 个body weights, HDL, } \\
\text { SOD GSH }\end{array}$ & $\begin{array}{l}\text { (Ma et al., } \\
2015)\end{array}$ \\
\hline Antimicrobial & C. cicadae S.Z. Shing & Hydroalcoholic & Agar well diffusion method & $\begin{array}{l}\text { Damage bacterial cell wall and } \\
\text { membranes, } \uparrow \text { cell permeability }\end{array}$ & $\begin{array}{l}\text { (Zhang et al., } \\
\text { 2017) }\end{array}$ \\
\hline Antibacterial & C. heteropoda Kobayasi & Cicadapeptins I and I| & Agar disk/diffusion assays & $\begin{array}{l}\text { Inhibition zones against Bacillus } \\
\text { cereus ( } 13 \text { and } 12 \mathrm{~mm} \text { ) and } B \text {. } \\
\text { subtilis ( } 13 \text { and } 11 \mathrm{~mm} \text { ), Escher- } \\
\text { ichia coli ( } 16 \mathrm{~mm} \text { for both } \\
\text { peptides) }\end{array}$ & $\begin{array}{l}\text { (Krasnoff et al., } \\
2005)\end{array}$ \\
\hline \multirow[t]{2}{*}{ Antifungal } & & Cicadapeptins I and I| & Potato dextrose agar plates & $\begin{array}{l}\text { Botrytis cinereal (11 mm zones) } \\
\text { showed inhibitory activity }\end{array}$ & $\begin{array}{l}\text { (Krasnoff et al., } \\
2005)\end{array}$ \\
\hline & $\begin{array}{l}\text { C. dipterigena Berk. and } \\
\text { Broome }\end{array}$ & Cordycepsidone A & Gibberella fujikuroi & $\begin{array}{l}\text { Strong and dose-dependent } \\
\text { activity }\end{array}$ & $\begin{array}{l}\text { (Varughese } \\
\text { et al., 2012) }\end{array}$ \\
\hline
\end{tabular}


TABLE 2 | (Continued) Immunostimulatory and related bioactivities of Cordyceps spp.: in vitro and in vivo.

\begin{tabular}{|c|c|c|c|c|c|}
\hline Activity & Species & Extract/Compound & $\begin{array}{l}\text { In vitro/in } \\
\text { vivo }\end{array}$ & Results & References \\
\hline \multirow[t]{2}{*}{ Neuroprotective } & C. cicadae S.Z. Shing & $\begin{array}{l}\text { Polysaccharides, } \\
\text { adenosine }\end{array}$ & Glutamate-induced PC12 cells & $\begin{array}{l}\uparrow \text { Cell survival rate, GPx, SOD, } \\
\mathrm{Bcl}^{2} 2 / \mathrm{Bax} \text { ratio, } \downarrow R O S \text { and } \\
\mathrm{Ca}^{2+} \text {, ERK, p38, and JNK } \\
\text { expression }\end{array}$ & $\begin{array}{l}\text { (Olatunji et al., } \\
\text { 2016) }\end{array}$ \\
\hline & & Butanol fraction & Glutamate-induced PC12 cells & $\begin{array}{l}\downarrow R O S \text { accumulation, GSH-Px, } \\
\text { and SOD levels }\end{array}$ & $\begin{array}{l}\text { (Wang et al., } \\
\text { 2018) }\end{array}$ \\
\hline Antiviral & $\begin{array}{l}\text { C. guangdongensis T.H. Li, } \\
\text { Q.Y. Lin and B. Song }\end{array}$ & Aqueous & Influenza virus H9N2 & $\downarrow$ Pulmonary index by $22.1 \%$ & $\begin{array}{l}\text { (Yan et al., } \\
2010)\end{array}$ \\
\hline Antimalarial & C. brunnearubra BCC 1395 & Ethyl acetate & $\begin{array}{l}\text { Malarial parasite plasmodium } \\
\text { falciparum K1 }\end{array}$ & $\mathrm{IC}_{50}=18 \mu \mathrm{M}$ & $\begin{array}{l}\text { (lsaka et al., } \\
\text { 2007a) }\end{array}$ \\
\hline $\begin{array}{l}\text { Cannabinoid receptors } \\
\text { CB1 and CB2 (agonistic) }\end{array}$ & $\begin{array}{l}\text { C. annullata Kobayasi \& } \\
\text { Shimizu }\end{array}$ & Ethyl acetate & HEK293 cells & 15.5-75.5\% inhibition & $\begin{array}{l}\text { (Asai et al., } \\
\text { 2012) }\end{array}$ \\
\hline Anti-proliferative & $\begin{array}{l}\text { C. bassiana Z.Z. Li, C.R. Li, } \\
\text { B. Huang and M.Z. Fan }\end{array}$ & Ethanol & $\begin{array}{l}\text { VSMC and carotid artery of } \\
\text { balloon-injured rats }\end{array}$ & $\begin{array}{l}\downarrow \text { VSMC proliferation and } \uparrow E R K \\
1 / 2 \text { phosphorylation }\end{array}$ & $\begin{array}{l}(\mathrm{Jin} \\
2016)\end{array}$ \\
\hline Anti-trypanosomal & $\begin{array}{l}\text { C. cardinalis G.H. Sung \& } \\
\text { Spatafora }\end{array}$ & Methanol & Against trypanosoma brucei & $\mathrm{IC}_{50}=8.63 \mu \mathrm{g} / \mathrm{ml}$ & $\begin{array}{l}\text { (Umeyama } \\
\text { et al., 2014) }\end{array}$ \\
\hline Anti-fibrotic & C. cicadae S.Z. Shing & Ergosterol peroxide & NRK-49 F cell line & $\begin{array}{l}\text { Blockage of TGF- } \boldsymbol{\beta} 1 \text {-stimulated } \\
\text { phosphorylation of ERK1/2, p38 } \\
\text { and JNK pathway, } \downarrow \text { TGF- } \boldsymbol{\beta} 1 \text { - } \\
\text { induced fibroblasts }\end{array}$ & $\begin{array}{l}\text { Zhu R et al. } \\
\text { (2014) }\end{array}$ \\
\hline Anti-atopic dermatitis & $\begin{array}{l}\text { C. bassiana Z.Z. Li, C.R. Li, } \\
\text { B. Huang and M.Z. Fan }\end{array}$ & Butanol fraction & $\begin{array}{l}\text { Topical use of DNFB in NC/Nga } \\
\text { mice }\end{array}$ & $\begin{array}{l}\text { Blockade of histamine release, } \\
\text { lgE production, IL-4, and IFN- } \gamma \\
\text { secretion }\end{array}$ & $\begin{array}{l}\text { Wu G et al. } \\
(2011)\end{array}$ \\
\hline Pro-apoptotic & & $\begin{array}{l}\text { 4-Isopropyl-2-(1-phenyl- } \\
\text { ethyl) aniline }\end{array}$ & $\begin{array}{l}\text { MDA-MB-231, HeLa, and C6 } \\
\text { glioma cells }\end{array}$ & $\begin{array}{l}\downarrow \text { Proliferation of MDA-mb-231, } \\
\text { HeLa, and C6 glioma cells, re- } \\
\text { duced the phosphorylation of } \\
\text { STAT3, Src, and PI3K/p85 }\end{array}$ & $\begin{array}{l}\text { Kim M S et al. } \\
(2015)\end{array}$ \\
\hline Antitubercular & $\begin{array}{l}\text { Ophiocordyceps Communis } \\
\text { Hywel-Jones and Samson }\end{array}$ & Cordycommunin & $\begin{array}{l}\text { Mycobacterium tuberculosis } \\
\text { H37Ra }\end{array}$ & $\begin{array}{l}\mathrm{MIC}=15 \mu \mathrm{M}, \text { weak cytotoxicity } \\
\text { to } \mathrm{kB} \text { cells }\end{array}$ & $\begin{array}{l}\text { (Haritakun } \\
\text { et al., 2010) }\end{array}$ \\
\hline Anti-fatigue & $\begin{array}{l}\text { C. guangdongensis T.H. Li, } \\
\text { Q.Y. Lin and B. Song }\end{array}$ & Ethanol & Forced swimming & $\downarrow$ Blood lactic acid levels & $\begin{array}{l}\text { (Yan et al., } \\
2013)\end{array}$ \\
\hline $\begin{array}{l}\text { Protein tyrosine phos- } \\
\text { phatase inhibitor }\end{array}$ & C. gracilioides Kobayasi & $\begin{array}{l}\text { Terreusinone A, pinophi- } \\
\text { lin } C \text { and cryptosporiop- } \\
\text { tide A }\end{array}$ & $\begin{array}{l}\text { PTP1B, SHP2, CDC25B, LAR } \\
\text { and SHP1 enzyme }\end{array}$ & $\mathrm{IC}_{50}=3.4-50 \mu \mathrm{g} / \mathrm{ml}$ & $\begin{array}{l}\text { (Wei et al., } \\
2015)\end{array}$ \\
\hline Antihyperlipidemic & C. militaris (L.) Fr. & Polysaccharides & HFD-induced & $\begin{array}{l}\downarrow \text { Blood and liver lipid, } \uparrow S G P T, \\
\text { and antioxidant activity }\end{array}$ & $\begin{array}{l}\text { Wang L et al. } \\
(2015)\end{array}$ \\
\hline Renoprotective & C. pruinosa Petch & Whole broth & LPS-induced renal cell injury & $\begin{array}{l}\downarrow R B F \text { and GFR, ED-1, GRP78, } \\
\text { Beclin-1 autophagy and TUNEL } \\
\text { apoptosis, } \quad \text { blood leukocyte } \\
\text { counts, plasma blood urea ni- } \\
\text { trogen and creatinine level }\end{array}$ & $\begin{array}{l}\text { Wu M F et al. } \\
\text { (2011) }\end{array}$ \\
\hline Anti-HIV-1 & & Cordysobin & HIV-1 reverse transcriptase & $\mathrm{IC}_{50}=8.2 \times 10^{-3} \mu \mathrm{M}$ & $\begin{array}{l}\text { Wang SX et al. } \\
(2012)\end{array}$ \\
\hline Renoprotective & & Cyclosporine A & $\begin{array}{l}\text { Cyclosporine-induced renal tu- } \\
\text { bule dysfunction }\end{array}$ & $\begin{array}{l}\downarrow \text { Apoptosis, caspase-3 activa- } \\
\text { tion, } \uparrow \text { magnesium reabsorption } \\
\text { channels TRMP6 and TRMP7 }\end{array}$ & $\begin{array}{l}\text { (Chyau et al., } \\
\text { 2014) }\end{array}$ \\
\hline Anti-asthmatic & $\begin{array}{l}\text { C. sphecocephala (Klotzsch } \\
\text { ex Berk.) Berk. and M.A. } \\
\text { Curtis }\end{array}$ & Culture filtrate & $\begin{array}{l}\text { Ovalbumin-induced asthmatic } \\
\text { mice }\end{array}$ & $\begin{array}{l}\downarrow I L-4, I L-13 \text {, and } I L-25 \text { expres- } \\
\text { sion and undesirable immune } \\
\text { responses }\end{array}$ & $\begin{array}{l}(\text { Heo et al., } \\
2010)\end{array}$ \\
\hline Antiaging & C. sinensis (Berk.) Sacc. & Aqueous & D-galactose-induced aging & $\begin{array}{l}\uparrow S O D \text {, catalase, GSH, } \downarrow \text { MDA, } \\
\text { monoamine oxidase }\end{array}$ & $\begin{array}{l}\text { Chen et al. } \\
(1997)\end{array}$ \\
\hline
\end{tabular}


immunopotentiation in immunodeficient patients ( $\mathrm{Xu}$ et al., 1992). Interestingly, the induction of macrophages and the intestinal immune system in mice by oral administration of hot water decoction from cultured mycelia of $C$. sinensis has also been reported (Koh et al., 2002). They inferred modulatory IL-6 production by activating macrophages and enhance secretion of hematopoietic growth factors like granulocytemacrophage colony-stimulating factor (GM-CSF) and IL-6 from Peyer's patch cells (mainly composed of T and B cells) (Koh et al., 2002). Cordyceps spp. induced modulation of cytokines has been reported by others as well (Yu et al., 2004). C. sinensis play an immunomodulatory role in the pathogenesis of GAS (Group A Streptococcus) infection in U937 cells by inducing the expression of cytokines like IFN- $\gamma$, IL-12, and TNF- $\alpha$, that eventually augmented the phagocytosis (Kuo et al., 2007). C. militaris (L.) Fr. polysaccharides (CMP) induced immune activation were studied in cyclophosphamide-induced immunosuppressed mice by assessing the lymphocyte proliferation, phagocytic index, and other biochemical parameters (Wang $\mathrm{M}$ et al., 2012), thus hinting its use as a future immunomodulatory agent.

The immuno-stimulatory action of a compound is explicated by its competence to trigger the immune system of the living organism through inducing or activating its components. Numerous species of Cordyceps spp. exhibits immuno-stimulatory activities in distinct parts of the body (Table 2). The use of $C$. sinensis has been documented in the medicament of respiratory infections by activating the immune response via innate immunity promotion (Lin and Li, 2011). Cordyceps spp. also promotes the adaptive immune system, comprising the cellular and humoral immunity (Lin and Li, 2011). Zhu et al. (2012b) investigated the role of $C$. gunnii (Berk.) Berk.-derived polysaccharides for immunostimulatory and antitumor purposes, and cytokines expression in normal, immuno-compromized, and H22-bearing mice. They inferred that the polysaccharides from the C. gunnii (Berk.) Berk. probably boost the non-specific immunity, humoral and cellular immunity, and restrain the tumor growth. CP2-S, (a novel polysaccharide) purified from C. militaris (L.) Fr. exhibits immunostimulatory activity by inducing phagocytosis, NO production, respiratory burst, and secretion of IL- $1 \beta$ and IL-2 (from macrophages). Bi et al. (2018) reported the immunostimulatory action of the novel polysaccharide (lowmolecular-weight) obtained from the fruiting bodies (cultured) of C. militaris (L.) Fr. in splenic lymphocytes and natural killer cells through induction of MAPK, NF- $\mathrm{BB}$, and Toll-like receptor (TLR) two pathways. Ethanol extracts of $C$. sinensis enhance phagocytosis activity as evidenced by carbon clearance in tumor-bearing mice. It also caused a remarkable increment in an acid phosphatase activity and lysosomal enzymes in macrophages suggesting its antitumor action via the immuno-stimulating function (Shin et al., 2001; Shin et al., 2003).

\section{Anti-inflammatory Potential of Cordyceps spp.}

The extract (ethanolic) of cultured mycelia of C. militaris (L.) Fr. possess potent anti-inflammatory activity in the carrageenin-triggered edema and decrement in inducible nitric oxide synthase (iNOS) expression in macrophages. Since the synthesis of NO by iNOS is elevated in inflammatory ailments and leads to cellular injury, this activity confirms its anti-inflammatory action (Won and Park, 2005). In lipopolysaccharide (LPS)-induced macrophage, NO production was restrained by butanolic fraction of C. militaris (L.) Fr. and the chief component was cordycepin. It was inferred that cordycepin inhibited the phosphorylation of protein kinase $\mathrm{B}(\mathrm{Akt}), \mathrm{I} \kappa \mathrm{Ba}$, and $\mathrm{p} 38$. It also suppressed TNF- $\alpha$, cyclooxygenase-2 (COX-2), iNOS, and $\mathrm{NF}-\kappa \mathrm{B}$ translocation in these macrophages. Thus, hinted at the use of cordycepin for inflammation-linked disorders (Kim et al., 2006). C. sinensis has been reported to strengthen the cell-mediated immunity as well (Liu et al., 2007).

Interestingly, others have reported the application of $C$. sinensis as a cost-effective immunosuppressive agent after renal transplantation without obvious adverse effects ( $\mathrm{Li}$ et al., 2009). Moreover, cordycepin and C. sinensis regulates the functions of human immune cells in vitro by promoting the expression of IL-1 $\beta,-6,-8,-10$ and TNF- $\alpha$ of resting cells, and inhibited the phytohemagglutinin-induced expression of IL-2, -4, $-5,-12$ and IFN- $\gamma$ and TNF- $\alpha$. Furthermore, the cordycepin and C. sinensis treated human monocytic cell line (THP-1) exhibited a higher affinity for the transcription factors that are important in the gene regulation of various cytokines. Thus, cordycepin and $C$. sinensis regulates the immune cells via its immunoregulatory activity (Zhou et al., 2008). A heteropolysaccharide from cultured C. sinensis was reported to enhance the immunity in mice exposed to ionizing radiation by reducing oxidative injury and modulating the secretion of cytokines (IL-4, -5 and -17) (Zhang et al., 2011). It has been reported that methanolic fractions of $C$. sinensis contain ingredients having an immunosuppressive effect that inhibits blastogenesis, the activity of $\mathrm{NK}$ cell, and phytohemagglutinin induced IL-2 and TNF- $\alpha$ production by human mononuclear cells (Kuo et al., 1996). The crude extract and partially purified fractions of $C$. sinensis inhibit the generation of superoxide anion and release of elastase. Further, it was revealed that five constituents, cordysinins A-E accounted for these actions (Yang et al., 2011). The treatment of macrophages with diverse concentrations of C. militaris (L.) Fr. fruiting bodies (hot water extract) has potent suppressive effects on the production of these inflammatory mediators as evident by LPS-induced NO production, TNF- $\alpha$, and IL-6 secretion (Jo et al., 2010).

Similar results were also reported in another study where the immune activation by CMP was improved. Moreover, CMP increased the thymus and spleen indices, the spleen lymphocyte activity, immunoglobulin G (IgG) function, and the total quantity of white blood cells in mice serum. CMP also enhanced the expression of IFN- $\gamma$, TNF- $\alpha$, and IL- $1 \beta$ mRNA (Liu et al., 2016). Anti-inflammatory effects of another species of Cordyceps spp. i.e. C. bassiana Z.Z. Li, C.R. Li, B. Huang and M.Z. Fan was investigated (Kim T W et al., 2014). Its butanolic fraction showed the most effective anti-inflammatory response against LPS-activated RAW 264.7 macrophages by inhibiting I $\mathrm{kB} / \mathrm{NF}-\kappa \mathrm{B}$ pathway and suppressing p38 and $\mathrm{c}$-Jun 
TABLE 3 | Mechanism of action of the Cordyceps spp.-induced pharmacological activities.

\section{Pharmacological activity}

Mechanism of action

Immunomodulatory and anti-inflammatory activities

Antioxidant and antiaging activity

Antitumor effects

Hypoglycemic activity
- Augmented the in vivo and in vitro NK activities phagocyte reactions via the activation of macrophages

- Modulation of IL-6 production activated macrophages and enhance secretion of hematopoietic growth factors such as GM-CSF

-Modulation of cytokines

-Increase in an acid phosphatase activity, representing lysosomal enzymes, in macrophages

-Inducing the expression of cytokines like IFN- $\gamma$, IL-12, and TNF- a

-Increased TNF- $\alpha$ and IFN- $\gamma$, enhanced NO production, and induced iNOS mRNA and protein expressions in macrophage. Induction of mRNA expression of IL-1 $\beta, I L-6, I L-10$, and TNF- $\alpha$

-Modulation of transcription factors involved in the gene regulation of various cytokines.

-Upregulated the expression of TNF- $\alpha$, IFN- $\gamma$, IL-6, and IL-1 $\beta$

-Stimulate NO production, phagocytosis, respiratory burst activity, secretion

of $\mathrm{IL}-1 \beta$ and IL-2 of macrophages

-The decrement in iNOS expression in macrophages

-Inhibited the phosphorylation of Akt, $1 \kappa B \alpha$, and p38. Suppressed TNF- $\alpha$, COX-2, iNOS, and translocation of NF-kB in macrophages

-Inhibitory effects on the production of inflammatory mediators

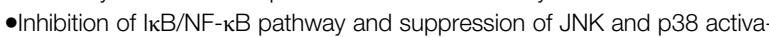
tion

-Suppresses the production NO, iNOS, and pro-inflammatory cytokines in macrophages via inhibition of NF- $\mathrm{kB}$ and $\mathrm{AP}-1$

- Inhibited MDA formation, anti-lipid peroxidation action and inhibited the accumulation of cholesteryl ester in macrophages

- Attenuating the changes of GPx and SOD activities, inhibited MDA formation -Modulating antioxidation activity via significantly enhancing SOD activity of liver, brain, and serum as well as GPx activity of liver and brain in tumorbearing mice

-Improve the activity of SOD of RBCs, brain and liver, the activity of na ${ }^{+}-\mathrm{K}^{+}$ATPE of the brain, the activity of catalase and GPx of blood, decrease the activity of monoamine oxidase of the brain and the contents of MDA of brain and liver in aged mice.

-Improved the activity of SOD, glutathione peroxidase and catalase and lowered the level of lipid peroxidation and monoamine oxidase activity

- Via immunomodulation

- Stimulating adenosine A3 receptors, Wnt signaling pathway, GSK3 $\beta$ activation cyclin D1 inhibition

-Caspase activation and mitochondrial dysfunction

- Via mTOR and AMPK signaling

-Enhancing JNK and p38 kinase activity and activity of Bcl-2 pro-apoptotic molecules

-Downregulating MDR/HIF-1a via AMPK/mTORC1 signaling

-Antiangiogenic via inhibiting tube formation in endothelial cells and MMP reduction

-Regulating Bcl-2 family and caspase activity and inhibition of COX-2 and prostaglandin E2 accumulation

-Regulation of p85/Akt-dependent or GSK3 $\beta$-related caspase-3-dependent apoptosis

-Involvement of hedgehog, apoptosis, p53, and estrogen signaling

- Potentiated the activities of glucokinase, hexokinase and glucose-6-phosphate dehydrogenase

- Increased the activity of hepatic glucokinase, the decline in the protein content of facilitative GLUT2

-By enhancing insulin sensitivity and improving oral glucose tolerance

-Stimulates the expression of HNF-1 a to activate GLUT2 for glucose uptake, induced AMPK phosphorylation, and gluconeogenesis inhibition

-anti-PTP1B activity

\section{References}

(Xu et al., 1992; Koh et al., 2002; Shin et al., 2003; Yu et al., 2004; Won and Park, 2005; Chen et al., 2006; Kim et al., 2006; Kuo et al., 2007; Ohta et al., 2007; Zhou et al., 2008; Zhu L et al. (2014); Kim T W et al. (2014); Park et al., 2014; Yang et al., 2015a; Chiu et al., 2016b; Liu et al., 2016; Jung et al., 2019)

(Yamaguchi et al., 2000a; Gu et al., 2003; Wang et al., 2004; Chen et al., 2006; Ji et al., 2009; Wang M et al. (2012)

(Yoshida et al., 1989; Yoo et al., 2004; Park et al., 2005; Yoshikawa et al., 2007 ; Jin et al., 2008; Yoshikawa et al., 2008; He et al., 2010; Wong et al., 2010; Jen et al., 2011; Wu et al., 2014; Park et al., 2017; Lee et al., 2019)

(Kiho et al., 1996; Kiho et al., 1999; Balon et al., 2002; Zhao et al., 2002; Kim et al., 2017; Sun et al., 2019)

(Continued on following page) 
TABLE 3 | (Continued) Mechanism of action of the Cordyceps spp.-induced pharmacological activities.

Pharmacological activity

Mechanism of action

Hypocholesterolemic, hypotensive and vasorelaxation activities

Anti-fatigue and antidepressant activity level hepatic lipase $(\mathrm{HL})$ activity

-Increase in levels of serum insulin
- The endothelium-dependent vasorelaxant effect through stimulating the production of nitric oxide and endothelium-derived hyperpolarizing factor

- anti-lipid peroxidation activities and inhibit the accumulation of cholesteryl ester in macrophages via suppression of LDL oxidation

-Inhibiting LDL oxidation through scavenging free radicals

-Increased the HDL cholesterol level, but decreased VLDL LDL cholestero

-Inhibited PDGF-BB-induced RASMCs migration and proliferation via interfering with adenosine receptor-mediated NOS pathways

-Reduced serum total cholesterol, triglyceride, LDL-C, VLDL-C as well as LDL-C/HDL-C and TC/HDL-C ratios. Increase in lipoprotein lipase (LPL) and

-Reduction in the levels of blood and liver lipid, and improvement of the glutamate pyruvate transaminase and antioxidant activity

- Facilitating efficient oxygen utilization, enhance energy metabolism in the mitochondria

- The increasing level of $\boldsymbol{\beta}$-ATP

-Increased the metabolic threshold and the ventilatory threshold of the subjects

-Extended the exhaustive swimming time of mice, hepatic and muscle glycogen levels, and decrease the blood lactic acid and blood urea nitrogen (BUN) levels

-Upregulation of skeletal metabolic regulators AMPK, PGC-1 and PPAR- as well as activation of NRF-2-ARE pathway

-Reducing the accumulation of blood lactic acid level.

- Via decreasing MDA and 8-OHdG levels and increasing antioxidant enzymes activities (SOD, catalase and GPx) in the serum, liver and muscle of mice

-Activating AMPK and protein kinase B (AKT)/mammalian target of rapamycin (mTOR) pathways and regulating serum hormone level

- PKC, cAMP-protein kinase A signal pathway

- Induce the expression of steroidogenic acute regulatory (StAR) protein

-Induce in vivo plasma corticosterone level

-adenosine receptors activated cAMP-PKA-StAR pathway

-PLC/PKC and MAPK signal transduction pathways

- Stimulating CYP11A1, 3及-HSD, and CYP17A1 expressions

\section{References}

Yamaguchi et al., 2000a; Yamaguchi et al., 2000b; Chiou et al., 2000; Koh et al., 2003; Won et al., 2009; Gao et al., 2011; Guo et al., 2011; Wang L et al. (2015)

(Zhang et al., 1995; Xiao et al., 1999; Dai et al., 2001; Li and Li, 2009; Chen S et al. (2010); Kumar et al., 2011; Yan et al., 2012; Yan et al., 2013; Song et al., 2015; Geng et al., 2017)

Wang et al. (1998), Huang et al. (2000), Hsu et al. (2003a), Hsu et al. (2003b), Huang B M et al. (2004), Chen et al. (2005), Leu et al. (2005), Chen S et al. (2010), Leu et al. (2011), Pao et al. (2012), Wang et al. (2016)

Footnote: DC-SIGN, dendritic cell-specific intercellular adhesion molecule-3-grabbing non-integrin; ERK, 1/2 extracellular signal-related kinases one and two; IL, interleukin; JNK, c-jun NH2-terminal kinase; MAPK, mitogen-activated protein kinase; MGL, macrophage galactose-type C-type lectin; MR, mannose receptor; MyD88, myeloid differentiation primary response protein 88; NF-кB, nuclear factor 'kappa-light-chain-enhancer' of activated B cells; $P 38$, mitogen-activated protein kinase; PI3K, phosphatidylinositol 3-kinase; RAF, rapidly accelerated fibrosarcoma; RAS, rat sarcoma; SOCS, suppressor of cytokine signaling; SYK, Spleen tyrosine kinase; Th2, T helper type 2; TLR, toll-like receptor; Treg, regulatory T cells; TRIF, TIR domain-containing adapter inducing IFN- $\boldsymbol{\beta}$.

$\mathrm{N}$-terminal kinase (JNK) activation. Moreover, 4-quinolinol and 1-naphthol were found from C. bassiana as an anti-inflammatory compound.

Paecilomyces hepiali Q.T. Chen and R.Q. Dai, CBG-CS-2 strain, isolated from Cordyceps spp. was investigated for the anti-inflammatory effects (Park et al., 2014). It was documented that CBG-CS-2 downregulates the NO production, iNOS, and pro-inflammatory cytokines in LPSstimulated macrophages by inhibition of NF- $\mathrm{BB}$ and activating protein (AP)-1, which are important in inflammation. Thus, the modulatory activity of CBG-CS-2 on the inflammatory response in macrophages, makes it useful as an anti-inflammatory drug or supplement. They further extended their study to confirm the immunoregulatory efficacy and safety of CBG-CS-2 separated and cultivated from $P$. hepiali from $C$. sinensis in healthy Korean adults (Jung et al., 2019). The major components reported i.e. CBG-CS-2, cordycepin, Polysaccharides, and adenosine induce immunomodulation by enhancing both the NK-cell activity and phagocyte reactions via macrophages activation. Moreover, cerebrosides have been reported to account for the antiinflammatory activity of $C$. militaris (L.) Fr. namely cordycerebroside A, soyacerebroside I, and glucocerebroside (Chiu et al., 2016b). Summary of the factors involved in cordyceps-induced immunomodulatory and anti-inflammatory activity is depicted in Table 3. C. sinensis partially protected animal models of bacterial growth by activating macrophages. It can also induce the expression of IL- $1 \beta$, IL-10, TNF- $\alpha$, serum immunoglobulin IgG1, and IgG2b, as well as stimulates Th1 immune response using IFN- $\gamma$ and IL-12 (Kuo et al., 2001; Lee et al., 2006). Concerning anti-inflammatory effects, cordymin, a purified compound from $C$. sinensis exhibited a decline in IL-1 $\beta$, TNF- $\alpha$, and pro-inflammatory markers in a carrageenan-induced inflammation model. Complementarily, the extracted compounds cordymin-1, cordymin-2, and cordymin-4 
presented an antinociceptive effect in acetic acid-induced abdominal constrictions model (Qian et al., 2012). Similarly, the anti-inflammatory activity of $C$. sinensis extracts on the human neutrophils' response was verified by inhibiting superoxide anion and elastase release. Most of the compounds produced an anti-inflammatory response superior to the indomethacin control, reaching a concentration necessary for $50 \%$ inhibition of $0.45 \mu \mathrm{g} / \mathrm{ml}$ for superoxide anion generation, and $1.68 \mu \mathrm{g} / \mathrm{ml}$ for elastase release. While for indomethacin, 38.32 , and $31.98 \mu \mathrm{g} / \mathrm{ml}$, respectively, were required (Yang et al., 2011). In another more detailed report, Cordycepin inhibited the overproduction of $\mathrm{NO}$, prostaglandin $\mathrm{E}_{2}$, and pro-inflammatory cytokines in a dose-dependent manner on the production of inflammatory mediators in LPS-stimulated murine BV2 microglia. Those outcomes inferred that cordycepin has a high potential in restraining inflammatory mediators in neurodegenerative diseases (Jeong et al., 2010).

\section{Antiviral effects of Cordyceps spp.}

Intranasal administration of an acidic polysaccharide (APS), obtained from the extract of C. militaris (L.) Fr. cultivated on germinated soybeans, decreased the virus titers in the bronchoalveolar lavage fluid and the lung of mice infected with influenza A virus with increased survival rate. Furthermore, APS also increased TNF- $\alpha$ and IFN- $\gamma$ levels. It enhanced NO production and induced iNOS mRNA and protein expressions in RAW 264.7 murine macrophage cells. The induction of mRNA expression of cytokines including IL- $1 \beta$, IL-6, IL-10, and TNF- $\alpha$ demonstrated its beneficial therapeutic effects on influenza A virus infection by modulating immune function of macrophages (Ohta et al., 2007).

\section{Antioxidant and Antiaging Activity}

The antioxidative profile of ethanol and water extract of $C$. sinensis (cultured) was assessed and initiated to be minimal on superoxide but it moderately inhibited MDA (malondialdehyde) formation (Yamaguchi et al., 2000a). C. sinensis has anti-lipid peroxidation potential and inhibits cholesteryl ester accumulation in macrophages thru LDL oxidation destruction. Li et al. (2001b) described that the adenosine content in Cordyceps spp. has no apparent relationship with anti-oxidation potential but later they confirmed that polysaccharides have the antioxidation profile.

Further, they extended their studies (Gu et al., 2003) and isolated a polysaccharide $(210 \mathrm{kDa})$ from cultivated Cordyceps spp. mycelia having strong anti-oxidative activity. Thus, they concluded that Cordyceps spp. protects against neuronal cell toxicity. Chen et al. (Chen et al., 2006) informed that polysaccharide from $C$. sinensis probably inhibits tumor evolution mainly by modifying hosts' antioxidative action thru significantly enhancing SOD activity of brain, liver, and serum as well as GPx activity of liver and brain in tumor-bearing mice whereas, it remarkably reduces the MDA level in liver and brain (Chen et al., 2006).

$\mathrm{Wu}$ et al. performed an in vitro antioxidant activity of CMhsCPS2 (a polysaccharide) which was isolated from fruiting bodies of C. militaris (L.) Fr. grownup on solid rice medium.
(Wu F Y et al., 2011). Similarly, CBP-1 a novel polysaccharide was isolated from cultured C. militaris (L.) Fr. was testified to have the hydroxyl radical-scavenging power. Since these radicals are associated with the pathogenesis of several ailments, the study implicit for latent clinical applications of C. militaris (L.) Fr. as a substitute for C. sinensis in TCM (Yu et al., 2009).

Aging has been reported to involve oxidative stress by many researchers (Romano et al., 2010). A study by Wang et al., 2004 confirmed, that $C$. sinensis increases the capability of learning and memory, improve the action of SOD of RBC's, mind and liver, the action of $\mathrm{Na}^{+}-\mathrm{K}^{+}$-ATPE of the brain, the potential of catalase and GPx of blood, and remarkably decline the activity of monoamine oxidase of the brain and the contents of MDA of brain and liver in aged mice by improving the antioxidative profile and eradicating free radicals (Wang et al., 2004). Ji et al. treated (Ji et al., 2009) D-galactose-induced senescence mice with $C$. sinensis extract. The results documented that $C$. sinensis extract can ameliorate the brain function and possess antioxidant activity by improving the activity of SOD, GPx, and catalase as well as lower the level of lipid peroxidation and monoamine oxidase. Another species i.e. C. guangdongensis has already been stated to have noteworthy antioxidative stress properties (Zeng et al., 2009). Another study with C. guangdongensis showed that it prolongs the mean lifespan and the half-death time of fruit flies in lifespan tests (Yan et al., 2011).

Structural and antioxidant analysis of W-CBP50, W-CBP50 I, and W-CBP50 II polysaccharides (from cultured C. militaris (L.) Fr.) was performed and all of them exhibited significant antioxidative strength (Chen X et al., 2013). Four polysaccharide fractions (CMP-1, CMP-2, CMP-3, and CMP4) were extracted from cultured C. militaris (L.) Fr. depicted noticeable concentration-dependent antioxidant activities (Chen and Huang, 2014). Similarly, a novel low-molecular-weight polysaccharide (CMP-1) was isolated by Jing et al. (Jing et al., 2014) from the cultured C. militaris (L.) Fr. showed free radicalscavenging effects. The same group further isolated a novel polysaccharide (CMPA90-1; compound 1) from the cultured fruiting bodies of C. militaris (L.) Fr. that exhibited free-radicalscavenging effects (Jing et al., 2015). Summary of the factors involved in cordyceps-induced antioxidant and antiaging activity is depicted in Table 3.

\section{Antitumor Effects}

Many genera of Cordyceps spp. (natural or cultured) has been documented to display the capability to restrain the growth of tumors due to various bioactive compounds present for e.g. polysaccharides, sterols, and adenosine (Yoshida et al., 1989; Bok et al., 1999; Li and Wang, 2008; Zhou et al., 2009). The glycosylated ergosterol from the methanolic extract of $C$. sinensis was reported as a remarkable antiproliferative compound against various tumor cell lines (Bok et al., 1999). Moreover, the water extract of $C$. sinensis also accelerates the Kupffer cells mediated phagocytosis to prevent metastasis (Nakamura et al., 1999). Since Cordyceps spp. can be cultivated artificially, it was documented in a comparative study that as compare to natural Cordyceps spp., the cultivated fungus has stronger antitumor activity against MCF-7, B16, HL-60, and HepG2 cancer cell lines (Zhang Q et al., 2005). 
Cordycepin restrains the proliferation of cancer cells by triggering adenosine A3 receptors followed by the Wnt signaling pathway, including glycogen synthase kinase three beta (GSK3 $\beta$ ) activation and cyclin D1 inhibition (Yoshikawa et al., 2004, 2007; Yoshikawa et al., 2008). In another study on MA-10 mouse Leydig tumor cell, cordycepin induced apoptosis was reported to involve caspase $-9,3$, and -7 dependent pathway (Jen et al., 2011). Moreover, the antiproliferative response of cordycepin is documented to be mediated via the mammalian target of rapamycin (mTOR) and $5^{\prime} \mathrm{AMP}$-activated protein kinase (AMPK) signaling (Wong et al., 2010). In human colorectal cancer cells, cordycepin triggers apoptosis via increasing B-cell lymphoma 2 (Bcl-2, proapoptotic molecules), JNK, and p38 kinase activity (He et al., 2010). As an adjuvant, a low concentration of cordycepin enhances the chemosensitivity of gall bladder cancer cells for gemcitabine and 5-fluorouracil, possibly via downregulating multiple drug-resistant/hypoxiainducible factor 1 (MDR/HIF-1 $\alpha)$ through regulating AMPK/ mTORC1 signaling (Wu et al., 2014). Thus, it can be inferred that cordycepin induced antitumor profile involves plethora of pathways depending upon the cell type. Ji et al. reported the co-effect of fermented C. sinensis and selenium on uterine cervix cancer, where they reported that this combination attenuates the oxidative stress and refine the immune function as compared to their effect (Ji et al., 2014).

Aqueous extract from another species, C. militaris (L.) Fr. showed cytotoxic profile against stomach adenocarcinoma (SNU1); colorectal adenocarcinoma (SUN-C4); and hepatocellular carcinoma (SNH-354), where cordycepin was reported as an active component (Lim et al., 2004). Extract of C. militaris (L.) Fr. possesses antiangiogenic properties as evident via inhibition of tube formation in endothelial cells and matrix metallopeptidase (MMP) reduction, a factor related to metastasis and invasion (Yoo et al., 2004). Similarly, C. militaris (L.) Fr. induces apoptosis via mitochondrial dysfunction and caspase activation in human breast cancer cell lines as well (Jin et al., 2008). Furthermore, pure compounds isolated from the extracts of $C$. militaris (L.) Fr. have been reported to be antiproliferative against PC-3, colon 205, and HepG2 cells (Rao et al., 2010). Furthermore, it was reported that C. militaris (L.) Fr. inhibit cancer growth through regulation of p85/Akt-dependent or GSK3 $\beta$-related caspase-3-dependent apoptosis on a xenograft mouse model bearing murine $\mathrm{T}$ cell lymphoma (RMA) cell-derived cancers (Park et al., 2017).

It has also been documented that $C$. sinensis inhibits tumorcell proliferation activities in different types of cancer cell lines, such as Jurkat, HepG2, PC 3, Colon 205, and MCF-7 (Rao et al., 2007). C. militaris (L.) Fr. concentrate and cordycepin elicit apoptosis via caspase- $7,-8$, and -9 involving the increase of $\mathrm{Bcl}-2$-associated $\mathrm{x}$ protein $(\mathrm{Bax}) / \mathrm{Bcl}-2$ protein expression ratio and decreasing $\mathrm{X}$-linked inhibitor of apoptosis protein (XIAP) thus confirming its anti-cancer property (Lee et al., 2019). Cordycepin exhibited an anti-cancer effect against B16 mouse melanoma by inducing the adenosine $\mathrm{A} 3$ receptor, and eventual activation of glycogen synthase kinase- $3 \beta$, and the suppression of cyclin $\mathrm{D}_{1}$. Furthermore, cordycepin exerts a coadjuvant effect with other drugs, as demonstrated when combined with $2^{\prime}$ deoxycoformycin increased three hundred-fold the anti-cancer effect in B16 cells (Nakamura et al., 2015). Other mechanisms that describe the anti-cancer activity of Cordyceps spp. involve apoptosis and autophagy, as depicted in $\mathrm{LNCaP}$ (human prostate carcinoma) cells. Furthermore, the autophagy mechanism was evident by the increase and accumulation of microtubuleassociated protein light chain-3 (LC3) (Lee et al., 2014). A summary of the factor involved in cordyceps-induced antitumor activity is depicted in Table 3.

\section{Hypoglycemic Activity}

Kiho et al. (1993) displayed that polysaccharides obtained from the cultivated mycelium of C. sinensis (CS-F30) lower the plasma glucose level in normal and streptozotocin (STZ) induced diabetic mice by intraperitoneal administration in comparison to slight lowering through oral administration. Additionally, they also verified that CS-F30 potentiate the activities of glucokinase, hexokinase, and glucose-6-phosphate dehydrogenase thus accelerating the glucose metabolism, which in turn was responsible for its antidiabetic activity (Kiho et al., 1996). Kiho et al. (1993) also presented the intraperitoneal administration of CS-F10, a polysaccharide purified from hot water extract of cultured mycelium of $C$. sinensis, on normal, STZ-induced diabetic and epinephrine-induced hyperglycemic mice lowered the plasma glucose level and increased the activity of hepatic glucokinase. An industrial fermentation product i.e. CordyMax ${ }^{\mathrm{TM}}$ Cs-4 gained by a proprietary mycelial strain from natural $C$. sinensis, is described to be effective in lowering basal blood glucose and plasma insulin. Additionally, it improves the metabolism of glucose by increasing insulin sensitivity and improving oral glucose tolerance (Balon et al., 2002; Zhao et al., 2002). A study confirmed that the Cordyceps spp. has a hypoglycemic activity in nicotinamide (NA) and STZ-induced diabetic rats as evident by attenuation of the polydipsia, hyperglycemia, and weight loss (Lo et al., 2004). The extract from $C$. sinensis has been documented to promote $\beta$-cell survival in the diabetes mellitus-II mouse model. (Kan et al., 2012).

C. sinensis has already been stated to give a shielding effect on podocytes in rats with diabetic nephropathy (Hao et al., 2014). In the same way, CmNo1, a novel combination of the fruiting body and C. militaris (L.) Fr. mycelia, has also been testified to deliver renoprotection in high-fat diet and STZ-NA-induced diabetic (type 2) mice (Yu et al., 2016). Kim et al. in a study concluded that C. militaris (L.) Fr. water extract (CMW) stimulates the expression of hepatocyte nuclear factor (HNF)-1 $\alpha$ to activate GLUT2 for glucose uptake in liver cells. Recently a study to isolate and characterize cerebrosides with anti- PTP1B activity from $C$. militaris (L.) Fr. was performed. The results documented that all four cerebrosides obtained from C. militaris (L.) Fr. exhibited inhibitory activity against PTP1B (Sun et al., 2019). A summary of the factors involved in cordyceps-induced hypoglycemic activity is depicted in Table 3.

\section{Hypocholesterolemic, Hypotensive and Vasorelaxation Activities}

In previous studies, the existence of a protein component in $C$. sinensis is reported to diminish the mean arterial pressure of rats 
and induce a direct endothelium-dependent vasorelaxant effect through stimulating the production of NO and endotheliumderived hyperpolarizing factor. They reported the effect to be triggered by a single active constituent or by the combined action of many agents found in the extract that contributes to hypotensive and vasorelaxation activities (Chiou et al., 2000). Besides antioxidant profile, C. sinensis possess potent anti-lipid peroxidation activities and prevent the accumulation of cholesteryl ester in macrophages via suppression of LDL oxidation (Yamaguchi et al., 2000a).

Yamaguchi et al. (Yamaguchi et al., 2000b) performed the effect of the water extract from cultured CMW on serum lipid and lipid peroxide levels and aortic cholesterol accumulation using an atherosclerosis mouse model and concluded that CMW prevents cholesterol deposition in the aorta by impeding LDL oxidation via scavenging free radicals. Remarkably, a study was completed (Won et al., 2009) to determine the effect of cordycepin obtained by C. militaris (L.) Fr., on responses of rat aortic smooth muscle cells (RASMCs) and vascular disorders, especially neointimal formation. It was documented that cordycepin inhibited platelet-derived growth factor-BB (PDGF-BB)-induced RASMCs migration and proliferation via interfering with adenosine receptor-mediated NOS pathways, thus resulting in the attenuation of neointima formation and thus could act as atherosclerosis agent. Furthermore, an increase in lipoprotein lipase (LPL) and hepatic lipase (HL) activity by cordycepin hint its contribution to lipid profiles regulation with no toxicity (Gao et al., 2011).

In a contemporary treatment approach toward both diabetes and depression management by vanadium-enriched C. sinensis (VECS). It was reported that in STZ-induced hyperglycemic rats administration of VECS, significantly reduces the blood glucose levels with the increase in levels of serum insulin (Guo et al., 2011). The study also revealed a remarkable decrease in immobility with a corresponding increase in the swimming and climbing behavior in hyperglycemic rats following VECS treatment thus concluding a contemporary treatment approach that advocates an aggressive stance toward both diabetes and depression management (Guo et al., 2011). Wang L et al. (2015) reported that the residual polysaccharide from C. militaris (L.) Fr. exhibited potential antihyperlipidemic, hepatoprotective, and antioxidant properties as depicted by the reduction in the levels of blood and liver lipid, and improvement of the glutamate pyruvate transaminase and antioxidant activity. Summary of the factors involved in Cordyceps-induced hypocholesterolemic, hypotensive and vasorelaxation activities is depicted in Table 3.

\section{Larvicidal Activity}

Due to its eco-friendly nature and less or no side effects of microbial metabolites as an insecticide, they are of great use (Berdy, 1989). Kim et al. (2002) reported that C. militaris (L.) Fr. fruiting body-derived cordycepin acts as a naturally occurring insecticide against Plutella xylostella L. larvae via direct effect rather than an inhibitory action of chitin synthesis and that this compound has stomach action.

\section{Anti-fatigue and Antidepressant Activity}

Cordyceps spp. has been used for ages as a medicine for increasing physical stamina to deal with weakness and fatigue by people of high altitude. Cordyceps spp. mushroom began to be in the spotlight in 1993, when some world athletics champions revealed part of their strategy for success, including a diet based on Cordyceps spp. ingredients (Kashyap et al., 2016). It works by an increase in cellular ATP increasing bioenergy and thus facilitating efficient oxygen utilization (Geng et al., 2017). Interestingly, athletes also use Cordyceps spp. to deal with fatigue and weakness thus increasing energy levels and extra endurance (Zhu et al., 1998). Dai et al. (2001) performed a study to evaluate the effects of CordyMax ${ }^{\mathrm{TM}} \mathrm{Cs}-4$, a mycelial fermentation product of $C$. sinensis, on energy metabolism. They documented that CordyMax remarkably improved the bioenergy status in the murine liver by increasing the level of $\beta$-ATP (adenosine triphosphate). Thus, the study supported the energypromoting properties of CordyMax. As discussed above, the antioxidant properties of Cordyceps spp. enhance energy metabolism in the mitochondria and facilitate the efficient utilization of limited oxygen supply, thus increasing the anaerobic threshold (Zhang et al., 1995; Xiao et al., 1999). Since it has been well-established that fatigue is closely related to depression, a study was performed using the tail suspension test in mice to examine the antidepressant effects of supercritical fluid extract (SCCS) of C. sinensis. Results suggest that SCCS may elicit an antidepressant-like effect by affecting the adrenergic and dopaminergic systems, but not by affecting the serotonergic system (Nishizawa et al., 2007). To examine the effect of Cs-4 on erobic capacity in healthy elderly volunteers a double-blind, placebo-controlled trial was performed (Chen S et al., 2010). It was documented that administration of Cs-4 for 12 weeks, increased the metabolic threshold and the ventilatory threshold of the subjects. Such higher thresholds indicate better erobic performance without fatigue in older human subjects.

The effects of polysaccharides from $C$. sinensis mycelium on physical fatigue in mice documented that $C$. sinensis polysaccharides extended the exhaustive swimming time of mice, hepatic and muscle glycogen levels, and decrease the blood lactic acid and blood urea nitrogen (BUN) levels. Such observations confirmed the anti-fatigue effects of $C$. sinensis polysaccharides ( $\mathrm{Li}$ and Li, 2009; Yan et al., 2012). To explore the underlying mechanisms behind the exercise endurance promoting activities of C. sinensis, Kumar et al. (2011) reported that such beneficial effects are mediated by upregulation of skeletal metabolic regulators AMPK, peroxisome proliferator-activated receptor gamma (PGC)-1 and peroxisome proliferator-activated receptors (PPAR)- as well as activation of NF-E2-related factor 2 (NRF-2)antioxidant responsive element (ARE) pathway that reduces exercise-induced oxidative stress and inflammation. To other species, i.e. C. guangdongensis has also been reported that it exhibits anti-fatigue effect as evident by the longest swimming time in mice (Yan et al., 2011). Moreover, the active constituent accounting for C. guangdongensis induced anti-fatigue effect was 
reported to be a polysaccharide that alleviates fatigue by reducing the accumulation of blood lactic acid level (Yan et al., 2013).

Interestingly, it was reported that natural as well as laboratory cultured mycelia of $C$. sinensis can increase the motor coordination with improved metabolic and ventilatory that results in increased muscle endurance or antifatigue activity and mood elevator or antidepressant-like activity as a result of decreased endogenous depression (Singh et al., 2014). The antioxidative property of $C$. sinensis might be the reason for the increased skeletal muscle activity. Furthermore, C. militaris (L.) Fr. induces fatigue recovery is mainly through activating AMPK and AKT/mTOR pathways and regulating serum hormone level (Song et al., 2015). Summary of the factors involved in Cordyceps-induced anti-fatigue and antidepressant activity is depicted in Table 3.

\section{Aphrodisiac Potential}

Because Cordyceps spp. is a benchmark for a highly energetic source, its applications as a sexual stimulant and in sexual dysfunction are attractive (Zhu et al., 1998; Tuli et al., 2013a; Chen et al., 2017), even popularly known as the Himalayan Viagra (Kashyap et al., 2016). Cordyceps spp. modulates the release of sexual hormones such as testosterone, estrogen, and progesterone, controlling reproductive activity, and restoring the impaired functions (Sohn et al., 2012). Mechanistically Cordyceps spp. stimulates steroidogenesis through PKA and PKC signal transduction pathways, testosterone production, and plasma testosterone levels, even in sexually inactive murine models (Huang et al., 2001; Chen et al., 2005). One study described that $C$. sinensis promoted prostate cancer cells grown in mice by enhancing testosterone production and androgen receptor expression (Ma et al., 2018). Hsu et al. (2003a) explored the effect of $C$. sinensis and its extracted fractions on testosterone secretion in mice using in vivo and in vitro approaches. Another research by Huang Y L et al. (2004) documented the effects of $C$. sinensis and its fractions on steroidogenesis in mice, where they inferred the remarkable stimulation of testosterone production.

In particular, the administration of cordycepin can increase the weight of the epididymis, sperm motility, and movement, and the number of mature sperm (Kashyap et al., 2016), namely, the quality and quantity of the sperm. Wang et al. (1998) demonstrate that PKC may be responsible for the $C$. sinensis-induced steroidogenesis in primary rat adrenal cell cultures. C. sinensis also triggers the steroidogenesis process in primary mouse Leydig cell and induces dose-dependent apoptosis in MA-10 mouse Leydig tumor cells (Leu et al., 2011; Pan et al., 2011). Moreover, C. sinensis has been reported by Huang et al. (2000) to induce the expression of steroidogenic acute regulatory (StAR) protein, a critical protein for steroidogenesis, in MA-10 mouse Leydig tumor cells. The same group further extended their studies (Huang et al., 2001) for C. sinensis-induced steroidogenesis in normal Leydig cells and reported that it had different effects on hCG-stimulated steroidogenesis between normal vs. tumor cells. They documented that $C$. sinensis significantly stimulated testosterone production and new protein synthesis was required for steroidogenesis (Huang et al., 2001). These results were also supported by others (Hsu et al., 2003a; Huang Y L et al., 2004) who documented that $C$. sinensis and its extracted fractions could stimulate testosterone production in vitro and in vivo. Hsu et al. (2003b) further explored the regulatory mechanism of action of $C$. sinensis-induced steroidogenesis using inhibitors of PKA or PKC pathways in normal mouse Leydig cells. Results documented that $C$. sinensis activated the cAMPprotein kinase A signal pathway, but not protein kinase C, and attenuated P450 side-chain cleavage enzyme (P450scc) activity to reduce human chorionic gonadotropin-stimulated steroidogenesis in purified mouse Leydig cells (Hsu et al., 2003b). However, Chen et al. Chen et al. (2005) reported that the mechanisms underlying $C$. sinensis-stimulated steroidogenesis in MA-10 mouse Leydig tumor cells possibly go through the PKA and PKC pathways simultaneously. They further explored the mechanisms of $C$. sinensis-stimulated steroidogenesis and found that de novo protein synthesis, increased steroidogenic acute regulatory protein mRNA expression, a calcium signal, and a mitochondria electrochemical gradient were required for $C$. sinensisstimulated steroidogenesis (Chen S et al., 2010). Similarly, the effect of $C$. sinensis on the female reproductive system was also explored. It was showed that C. sinensis stimulates E2 production in human granulosa-lutein cells (GLCs) by upregulating the expression of several key enzymes, especially StAR and aromatase, making it a brilliant candidate for increasing the fecundity of women (Huang B M et al., 2004). Moreover, C. sinensis and its fractions have been reported to induce in vivo plasma corticosterone levels in immature and mature mice (Leu et al., 2005). Besides, C. sinensis could improve the function of reproduction and testis morphology in mice (Jin and Guo, 2006).

The in vitro effect of extracted fractions of $C$. sinensis mycelium on hCG-treated testosterone production from purified normal mouse Leydig cells was examined (Wong et al., 2007). It was reported that in normal mouse Leydig cells, all fractions of $C$. sinensis decreased hCG-stimulated testosterone production, which was opposite to the stimulatory effects of $C$. sinensis and fractions in tumor cells with hCG treatment. Different receptor subtypes between normal and tumor cells to activate different cellular functions are responsible for the difference (Huang et al., 1995; Huang et al., 1997). Administration of C. militaris (L.) Fr. mycelium improves sperm quality and quantity as evidenced by the improvement in the percentages of motile sperm cells and sperm morphology (Lin et al., 2007; Chang et al., 2008). Moreover, in vivo and in vitro effects of Cordycepin, were studied on primary mouse Leydig cell steroidogenesis. Cordycepin increased the plasma testosterone concentration as well as stimulated in vitro mouse Leydig cell testosterone production. It was reported that cordycepin associates with adenosine receptors to activate the cAMP-PKA-StAR pathway and steroidogenesis in the mouse Leydig cells (Leu et al., 2011).

Besides, others reported that cordycepin can stimulate progesterone production but also activate AR thus simultaneously induce steroidogenesis and apoptosis in MA-10 mouse Leydig tumor cells (Pan et al., 2011). Later intracellular 
phospholipase C/protein kinase C (PLC/PKC) and MAPK signal transduction pathways were reported to be responsible cordycepin induced steroidogenesis and cell death in MA10 mouse Leydig tumor cells (Pao et al., 2012). However, longterm administration of cordycepin can counteract the decline of testicular function in middle-aged rats (Sohn et al., 2012). C. militaris (L.) Fr. remarkably protect testicles against oxidative damage caused by bisphenol A, a commonly used plasticizer, and relieved degeneration of serum $\mathrm{T}$ and $\mathrm{LH}$ concentration caused by it, via stimulating Star, CYP11A1, 3 $\beta$-HSD, and CYP17A1 expressions (Wang et al., 2016). Summary of the factors involved in cordyceps-induced aphrodisiac potential is depicted in Table 3.

\section{Kidney Protection}

The kidney is the main organ responsible for filtering and eliminating waste through the production of urine. Among the various applications of the components of $C$. sinensis, one can also find its valuable use to regulate some imbalances of the kidney, for example for the reduction of hematuria and proteinuria with an evident restoration of the tissue evidenced by histological analysis (Ding et al., 2011). In addition to supporting kidney transplantation in combination with drugs such as cyclosporin A. That combination is beneficial because high doses of cyclosporin A can induce kidney damage (Ding et al., 2009). Likewise, C. sinensis exhibits nephroprotection properties to mitigate damage from aminoglycosides, broad-spectrum antibiotics (Bao et al., 1994; Heggers et al., 1996). These properties are associated with an increase of 17-hydroxy-corticosteroid, 17-ketosteroid, SOD enzymes, and free radical scavenging.

\section{TOXIC EFFECTS OF CORDYCEPS SPP.}

The array of secondary metabolites of polycyclic aromatic hydrocarbons (PAH) primarily developed from C. sinensis react with the polypropylene in popular bags, resulting in byproducts toxic to $C$. sinensis and spectacular progress over time. These polypropylene/PAH by-products inevitably damage the organism. To extend the growth period of the organism, C. sinensis must be cultured in glass or metal vessels (Holliday et al., 2004). The PAH compounds are present in the living culture, but they are volatile compounds and lost after drying. While Cordyceps spp. cannot usually be grown in polypropylene bags, new strains that produce considerably less PAH are designed to allow them to grow in plastic bags.

\section{CONCLUSIONS AND PROSPECTS}

Natural products are increasing the trust of people for the treatment and management of several chronic diseases. For hundreds of years, Cordyceps spp. have been used in Tibetan medicine and TCM, and in the last decades, the consumption of its fruiting bodies or related products as supplements has become popular. The most consumed and studied are $C$. sinensis and C. militaris (L.) Fr. Cordyceps spp. genus compromises a plethora of compounds and some of them showed therapeutic and pharmacological activities in preclinical studies, in vitro, and in vivo. Cordycepin and CA are important Cordyceps spp. bioactive constituents with important therapeutic applications associated with other compounds such as nucleotides, polysaccharides, cyclic peptides, sterols, and fatty acids are present in this genus and have shown a wide range of biological activities. The reservoir of Cordyceps spp. bioactive components show their therapeutic activities by modulating several cell signaling pathways due to the modulation of inflammation and oxidative/nitrosative stress processes. Cytokines releases, NO production via iNOS stimulation, and MAPK pathway are some of the cell signaling pathways modulated by Cordyceps spp. bioactive components. In the future, new chemical studies are needed to elucidate the unknown molecules present in Cordyceps spp., and new preclinical studies are needed to understand which compounds have the most interesting biological activities and the existing synergies between Cordyceps spp. components. Likewise, new drug formulations as nano drugs with cordycepin and other Cordyceps spp. biological compounds need to be developed and studied. However, new toxicological studies are needed to ensure their safety and promote its clinical studies. Clinical pilot studies with a few numbers of participants are needed as the first step to elucidate the potential of Cordyceps spp. as hypoglycaemic, hypocholesterolemic, and hypotensive agents. Other potential therapeutic effects such as anticancer may be more difficult to be elucidated in clinical studies and more pre-clinical studies are needed to a better understanding of the mechanisms involved. In conclusion, new future efforts are needed to elucidate the bioactive compounds present in Cordyceps genus and its therapeutic potential.

\section{AUTHOR CONTRIBUTIONS}

GD: Conceptualization, Methodology, Data curation, WritingOriginal draft preparation. H-SS: Methodology, WritingReviewing and Editing. GL-G: Data curation, WritingOriginal draft preparation, Writing- Reviewing and Editing. MP-A: Data curation, Writing- Original draft preparation. HC: Data curation, Writing- Original draft preparation. YS: Data curation, Writing- Original draft preparation, WritingReviewing and Editing. MP: Data curation, Writing- Original draft preparation. AM: Data curation, Writing- Original draft preparation, Writing- Reviewing and Editing. MN: Methodology, Data curation, Writing- Original draft preparation. SS: Data curation, Writing- Original draft preparation PC: Data curation, Writing- Original draft preparation. MM: WritingOriginal draft preparation, Writing- Reviewing and Editing. NM: Methodology, Data curation, Methodology, Data curation, Writing- Original draft preparation. VS: Writing- 
Original draft preparation. NG: Writing- Original draft preparation. RS: Writing- Original draft preparation. JKP: Visualization, Conceptualization, Methodology, Data curation, Writing- Original draft preparation, Software, WritingReviewing and Editing, Funding.

\section{FUNDING}

This work was supported by the National Research Foundation of Korea (NRF) grant funded by the Korea government (MSIT) (No. 2020R1G1A1004667), Republic of Korea.

\section{REFERENCES}

Asai, T., Luo, D., Obara, Y., Taniguchi, T., Monde, K., Yamashita, K., et al. (2012). Dihydrobenzofurans as cannabinoid receptor ligands from Cordyceps annullata, an entomopathogenic fungus cultivated in the presence of an HDAC inhibitor. Tetrahedron Lett. 53, 2239-2243. doi:10.1016/j.tetlet.2012. 02.088

Balon, T. W., Jasman, A. P., and Zhu, J.-S. (2002). A fermentation product of Cordyceps sinensis increases whole-body insulin sensitivity in rats. J. Alternative Compl. Med. 8, 315-323. doi:10.1089/107628002761574581

Bao, Z. D., Wu, Z. G., and Zheng, F. (1994). [Amelioration of aminoglycoside nephrotoxicity by Cordyceps sinensis in old patients]. Zhongguo Zhong Xi Yi Jie He Za Zhi. 14, 271-273.

Baral, B., Shrestha, B., and Teixeira De Silva, J. A. (2015). A review of Chinese Cordyceps with special reference to Nepal, focusing on conservation. Environ. Exp. Biol. 13, 61-73.

Berdy, J. (1989). The discovery of new bioactive microbial metabolites: screening and identification. Prog. Ind. Microbiol. 27, 3-27.

Bi, S., Jing, Y., Zhou, Q., Hu, X., Zhu, J., Guo, Z., et al. (2018). Structural elucidation and immunostimulatory activity of a new polysaccharide from Cordyceps militaris. Food Function 9, 279-293. doi:10.1039/C7FO01147D

Bok, J. W., Lermer, L., Chilton, J., Klingeman, H. G., and Towers, G. H. (1999). Antitumor sterols from the mycelia of Cordyceps sinensis. Phytochemistry 51, 891-898. doi:10.1016/s0031-9422(99)00128-4

Byeon, S. E., Lee, J., Yoo, B. C., Sung, G. H., Kim, T. W., Park, H. J., et al. (2011a). p38-targeted inhibition of interleukin-12 expression by ethanol extract from Cordyceps bassiana in lipopolysaccharide-activated macrophages. Immunopharmacol. Immunotoxicol. 33, 90-96. doi:10.3109/08923973.2010. 482137

Byeon, S. E., Lee, S. Y., Kim, A. R., Lee, J., Sung, G. H., Jang, H. J., et al. (2011b). Inhibition of cytokine expression by a butanol extract from Cordyceps bassiana. Pharmazie. 66, 58-62. doi:10.1691/ph.2011.0668

Chakraborty, S., Chowdhury, S., and Nandi, G. (2014). Review on yarsagumba (Cordyceps sinensis) - an exotic medicinal mushroom. Int. J. Pharm. Phytochem. Res. 6, 339-346.

Chang, Y., Jeng, K.-C., Huang, K.-F., Lee, Y.-C., Hou, C.-W., Chen, K.-H., et al. (2008). Effect of Cordyceps militaris supplementation on sperm production, sperm motility and hormones in Sprague-Dawley rats. Am. J. Chin. Med. 36, 849-859. doi:10.1142/S0192415X08006296

Chatterjee, R., Srinivasan, K. S., and Maiti, P. C. (1957). Cordyceps sinesis (Berkeley) Saccardo: structure of cordycepic acid. J. Am. Pharm. Assoc. 46, 114-118. doi:10.1002/jps.3030460211

Chen, J., Zhang, W., Lu, T., Li, J., Zheng, Y., and Kong, L. (2006). Morphological and genetic characterization of a cultivated Cordyceps sinensis fungus and its polysaccharide component possessing antioxidant property in $\mathrm{H} 22$ tumorbearing mice. Life Sci. 78, 2742-2748. doi:10.1016/j.lfs.2005.10.047

Chen, J.-L., Chen, Y.-C., Yang, S.-H., Ko, Y.-F., and Chen, S.-Y. (2009). Immunological alterations in lupus-prone autoimmune (NZB/NZW) F1 mice by mycelia Chinese medicinal fungus Cordyceps sinensis-induced redistributions of peripheral mononuclear T lymphocytes. Clin. Exp. Med. 9, 277-284. doi:10.1007/s10238-009-0043-3

\section{ACKNOWLEDGMENTS}

Authors are grateful to respective institutions for support. This work was supported by the National Research Foundation of Korea (NRF) grant funded by the Korea government (MSIT) (No. 2020R1G1A1004667), Republic of Korea. This work was also supported by the Dongguk University Research Fund of 2019. NCM acknowledges the Portuguese Foundation for Science and Technology under the Horizon 2020 Program (PTDC/PSI-GER/ 28076/2017). We would like to thank Naughty Dog for developing one of the best video games ever which is based on Cordyceps unilateralis.

Chen, P. X., Wang, S., Nie, S., and Marcone, M. (2013). Properties of Cordyceps sinensis: a review. J. Functional Foods 5, 550-569. doi:10.1016/j.jff.2013. 01.034

Chen, S., and Chu, J. (1996). NMR and IR studies on the characterization of cordycepin and 2'-deoxyadenosine. Chin. J. Antibiot. 21, 9-12.

Chen, S., Li, Z., Krochmal, R., Abrazado, M., Kim, W., and Cooper, C. B. (2010). Effect of Cs $-4^{\circledR}$ (Cordyceps sinensis) on exercise performance in healthy older subjects: a double-blind, placebo-controlled trial. J. Alternative Compl. Med. 16, 585-590. doi:10.1089/acm.2009.0226

Chen, W., Yuan, F., Wang, K., Song, D., and Zhang, W. (2012). Modulatory effects of the acid polysaccharide fraction from one of anamorph of Cordyceps sinensis on Ana-1 cells. J. Ethnopharmacol. 142, 739-745. doi:10.1016/j.jep.2012.05.055

Chen, W., Zhang, W., Shen, W., and Wang, K. (2010). Effects of the acid polysaccharide fraction isolated from a cultivated Cordyceps sinensis on macrophages in vitro. Cell. Immunol. 262, 69-74. doi:10.1016/j.cellimm.2010.01.001

Chen, X., Wu, G., and Huang, Z. (2013). Structural analysis and antioxidant activities of polysaccharides from cultured Cordyceps militaris. Int. J. Biol. Macromol. 58, 18-22. doi:10.1016/j.ijbiomac.2013.03.041

Chen, Y. C., Chen, Y. H., Pan, B. S., Chang, M. M., and Huang, B. M. (2017). Functional study of Cordyceps sinensis and cordycepin in male reproduction: a review. J. Food Drug Anal. 25, 197-205. doi:10.1016/j.jfda.2016.10.020

Chen, Y. C., and Huang, B. M. (2014). Regulatory mechanisms of Cordyceps sinensis on steroidogenesis in MA-10 mouse Leydig tumor cells. Biosci. Biotechnol. Biochem. 74, 1855-1859. doi:10.1271/bbb.100262

Chen, Y. C., Huang, Y. L., and Huang, B. M. (2005). Cordyceps sinensis mycelium activates PKA and PKC signal pathways to stimulate steroidogenesis in MA-10 mouse Leydig tumor cells. Int. J. Biochem. Cell Biol. 37, 214-223. doi:10.1016/j. biocel.2004.05.019

Chen, Y. J., Shiao, M. S., Lee, S. S., and Wang, S. Y. (1997). Effect of Cordyceps sinensis on the proliferation and differentiation of human leukemic U937 cells. Life Sci. 60, 2349-2359. doi:10.1016/s0024-3205(97)00291-9

Cheong, K.-L., Meng, L.-Z., Chen, X.-Q., Wang, L.-Y., Wu, D.-T., Zhao, J., et al. (2016). Structural elucidation, chain conformation and immuno-modulatory activity of glucogalactomannan from cultured Cordyceps sinensis fungus UM01. J. Functional Foods. 25, 174-185. doi:10.1016/j.jff.2016.06.002

Chihara, G., Maeda, Y., Hamuro, J., Sasaki, T., and Fukuoka, F. (1969). Inhibition of mouse sarcoma 180 by polysaccharides from lentinus edodes (berk.) sing. Nature. 222, 687-688. doi:10.1038/222687a0

Chiou, W. F., Chang, P. C., Chou, C. J., and Chen, C. F. (2000). Protein constituent contributes to the hypotensive and vasorelaxant acttvtties of Cordyceps sinensis. Life Sci. 66, 1369-1376. doi:10.1016/s0024-3205(00)00445-8

Chiu, C.-P., Hwang, T.-L., Chan, Y., El-Shazly, M., Wu, T.-Y., Lo, I. W., et al. (2016a). Research and development of cordyceps in Taiwan. Food Sci. Human Wellness. 5, 177-185. doi:10.1016/j.fshw.2016.08.001

Chiu, C.-P., Liu, S.-C., Tang, C.-H., Chan, Y., El-Shazly, M., Lee, C.-L., et al. (2016b). Anti-inflammatory cerebrosides from cultivated Cordyceps militaris. J. Agric. Food Chem. 64, 1540-1548. doi:10.1021/acs.jafc.5b05931

Chiu, C. H., Chyau, C. C., Chen, C. C., Lin, C. H., Cheng, C. H., and Mong, M. C. (2014). Polysaccharide extract of Cordyceps sobolifera attenuates renal injury in endotoxemic rats. Food Chem. Toxicol. 69, 281-288. doi:10.1016/j.fct.2014. 04.009 
Chou, S. M., Lai, W. J., Hong, T. W., Lai, J. Y., Tsai, S. H., Chen, Y. H., et al. (2014). Synergistic property of cordycepin in cultivated Cordyceps militaris-mediated apoptosis in human leukemia cells. Phytomedicine. 21, 1516-1524. doi:10.1016/ j.phymed.2014.07.014

Chyau, C. C., Chen, C. C., Chen, J. C., Yang, T. C., Shu, K. H., and Cheng, C. H. (2014). Mycelia glycoproteins from Cordyceps sobolifera ameliorate cyclosporine-induced renal tubule dysfunction in rats. J. Ethnopharmacol. 153, 650-658. doi:10.1016/j.jep.2014.03.020

Dai, G., Bao, T., Xu, C., Cooper, R., and Zhu, J.-S. (2001). CordyMax ${ }^{\mathrm{TM}}$ Cs-4 improves steady-state bioenergy status in mouse liver. J. Alternative Compl. Med. 7, 231-240. doi:10.1089/107555301300328106

Ding, C., Tian, P., Jia, L., Li, Y., Ding, X., Xiang, H., et al. (2009). The synergistic effects of C. Sinensis with CsA in preventing allograft rejection. Front. Biosci. 14, 3864-3871. doi:10.2741/3494

Ding, C., Tian, P. X., Xue, W., Ding, X., Yan, H., Pan, X., et al. (2011). Efficacy of Cordyceps sinensis in long term treatment of renal transplant patients. Front. Biosci. 3, 301-307. doi:10.2741/e245

Dou, Y., Xiao, J.-H., Xia, X.-X., and Zhong, J.-J. (2013). Effect of oxygen supply on biomass and helvolic acid production in submerged fermentation of Cordyceps taii. Biochem. Eng. J. 81, 73-79. doi:10.1016/j.bej.2013.10.005

Gao, J., Lian, Z. Q., Zhu, P., and Zhu, H. B. (2011). Lipid-lowering effect of cordycepin (3'-deoxyadenosine) from Cordyceps militaris on hyperlipidemic hamsters and rats. Yao Xue Xue Bao. 46, 669-676. PMID: 21882527.

Geng, P., Siu, K.-C., Wang, Z., and Wu, J.-Y. (2017). Antifatigue functions and mechanisms of edible and medicinal mushrooms. BioMed Res. Int. 2017, 1-16. doi:10.1155/2017/9648496

Gu, J.-Q., Li, W., Kang, Y.-H., Su, B.-N., Fong, H. H., Van Breemen, R. B., et al. (2003). Minor withanolides from Physalis philadelphica: structures, quinone reductase induction activities, and liquid chromatography (LC)-MS-MS investigation as artifacts. Chem. Pharmaceut. Bull. 51, 530-539. doi:10.1248/cpb.51.530

Guan, Y. J., Hu, Z., and Hou, M. (1992). Effect of Cordyceps sinesis on T-lymphocyte subsets in chronic renal failure. Zhongguo Zhong Xi Yi Jie He Za Zhi. 12, 338-339.

Guo, J., and Friedman, S. L. (2007). Hepatic fibrogenesis. Semin. Liver Dis. 27, 413-426. doi:10.1055/s-2007-991517

Guo, J., Li, C. Y., Wang, J., Liu, Y., and Zhang, J. (2011). Vanadium-Enriched Cordyceps sinensis, a contemporary treatment approach to both diabetes and depression in rats. Evid. base Compl. Alternative Med. 2011, 1-6. doi:10.1093/ ecam $/$ neq058

Han, E. S., Oh, J. Y., and Park, H. J. (2011). Cordyceps militaris extract suppresses dextran sodium sulfate-induced acute colitis in mice and production of inflammatory mediators from macrophages and mast cells. J. Ethnopharmacol. 134, 703-710. doi:10.1016/j.jep.2011.01.022

Hao, L. I., Pan, M.-S., Zheng, Y. U. N., and Wang, R.-F. (2014). Effect of Cordyceps sinensis and Tripterygium wilfordii polyglycosidium on podocytes in rats with diabetic nephropathy. Exp. Ther. Med. 7, 1465-1470. doi:10.3892/etm.2014. 1670

Haritakun, R., Sappan, M., Suvannakad, R., Tasanathai, K., and Isaka, M. (2010). An antimycobacterial cyclodepsipeptide from the entomopathogenic fungus ophiocordyceps communis BCC 16475. J. Nat. Prod. 73, 75-78. doi:10.1021/ np900520b

He, W., Zhang, M.-F., Ye, J., Jiang, T.-T., Fang, X., and Song, Y. (2010). Cordycepin induces apoptosis by enhancing JNK and p38 kinase activity and increasing the protein expression of Bcl-2 pro-apoptotic molecules. J. Zhejiang Univ. - Sci. B. 11, 654-660. doi:10.1631/jzus.B1000081

Heggers, J. P., Kucukcelebi, A., Listengarten, D., Stabenau, J., Ko, F., Broemeling, L. D., et al. (1996). Beneficial effect of aloe on wound healing in an excisional wound model. J. Alternative Compl. Med. 2, 271-277. doi:10.1089/acm.1996. 2.271

Heo, J. C., Nam, S. H., Nam, D. Y., Kim, J. G., Lee, K. G., Yeo, J. H., et al. (2010). Anti-asthmatic activities in mycelial extract and culture filtrate of Cordyceps sphecocephala J201. Int. J. Mol. Med. 26, 351-356. doi:10. 3892/ijmm_00000472

Holliday, J. C., Cleaver, P., Loomis-Powers, M., and Patel, D. (2004). Analysis of quality and techniques for hybridization of medicinal fungus Cordyceps sinensis (Berk.)Sacc. (Ascomycetes). Int. J. Med. Mushrooms. 6, 151-164. doi:10.1615/ intjmedmushr.v6.i2.60
Hsu, C.-C., Huang, Y.-L., Tsai, S.-J., Sheu, C.-C., and Huang, B.-M. (2003a). In vivo and in vitro stimulatory effects of Cordyceps sinensis on testosterone production in mouse Leydig cells. Life Sci. 73, 2127-2136. doi:10.1016/s0024-3205(03) 00595-2

Hsu, C.-C., Tsai, S.-J., Huang, Y.-L., and Huang, B.-M. (2003b). Regulatory mechanism of Cordyceps sinensis mycelium on mouse Leydig cell steroidogenesis. Fed. Eur. Biochem. Soc. Lett. 543, 140-143. doi:10.1016/ s0014-5793(03)00427-7

Hsu, T.-H., Shiao, L.-H., Hsieh, C., and Chang, D.-M. (2002). A comparison of the chemical composition and bioactive ingredients of the Chinese medicinal mushroom DongChongXiaCao, its counterfeit and mimic, and fermented mycelium of Cordyceps sinensis. Food Chem. 78, 463-469. doi:10.1016/ s0308-8146(02)00158-9

Huang, B. M., Chuang, Y. M., Chen, C. F., and Leu, S. F. (2000). Effects of extracted Cordyceps sinensis on steroidogenesis in MA-10 mouse Leydig tumor cells. Biol. Pharmaceut. Bull. 23, 1532-1535. doi:10.1248/bpb.23.1532

Huang, B. M., Hsiao, K. Y., Chuang, P. C., Wu, M. H., Pan, H. A., and Tsai, S. J. (2004). Upregulation of steroidogenic enzymes and ovarian $17 \beta$-estradiol in human granulosa-lutein cells by Cordyceps sinensis Mycelium1. Biol. Reprod. 70, 1358-1364. doi:10.1095/biolreprod.103.022855

Huang, B. M., Hsu, C. C., Tsai, S. J., Sheu, C. C., and Leu, S. F. (2001). Effects of Cordyceps sinensis on testosterone production in normal mouse Leydig cells. Life Sci. 69, 2593-2602. doi:10.1016/s0024-3205(01)01339-x

Huang, B. M., Stocco, D. M., Hutson, J. C., and Norman, R. L. (1995). Corticotropin-releasing hormone stimulates steroidogenesis in mouse Leydig cells. Biol. Reprod. 53, 620-626. doi:10.1095/biolreprod53.3.620

Huang, B. M., Stocco, D. M., and Norman, R. L. (1997). The cellular mechanisms of corticotropin-releasing hormone $(\mathrm{CRH})$-stimulated steroidogenesis in mouse Leydig cells are similar to those for LH. J. Androl. 18, 528-534.

Huang, L. F., Liang, Y. Z., Guo, F. Q., Zhou, Z. F., and Cheng, B. M. (2003). Simultaneous separation and determination of active components in Cordyceps sinensis and Cordyceps militarris by LC/ESI-MS. J. Pharmaceut. Biomed. Anal. 33, 1155-1162. doi:10.1016/s0731-7085(03)00415-1

Huang, L. F., Guo, F. Q., Liang, Y. Z., and Chen, B. M. (2004). [Determination of adenosine and cordycepin in Cordyceps sinensis and C. militarris with HPLCESI-MS]. Zhongguo Zhongyao Zazhi. 29, 762-764.

Huang, Y. L., Leu, S. F., Liu, B. C., Sheu, C. C., and Huang, B. M. (2004). In vivo stimulatory effect of Cordyceps sinensis mycelium and its fractions on reproductive functions in male mouse. Life Sci. 75, 1051-1062.

Hyun, S. H., Lee, S. Y., Sung, G. H., Kim, S. H., and Choi, H. K. (2013). Metabolic profiles and free radical scavenging activity of Cordyceps bassiana fruiting bodies according to developmental stage. PloS One. 8, e73065. doi:10.1371/ journal.pone.0073065

Ikeda, R., Nishimura, M., Sun, Y., Wada, M., and Nakashima, K. (2008). Simple HPLC-UV determination of nucleosides and its application to the authentication of Cordyceps and its allies. Biomed. Chromatogr. 22, 630-636. doi:10.1002/bmc.980

Isaka, M., Boonkhao, B., Rachtawee, P., and Auncharoen, P. (2007a). A xanthocillin-like alkaloid from the insect pathogenic fungus Cordyceps brunnearubra BCC 1395. J. Nat. Prod. 70, 656-658. doi:10.1021/np060509t

Isaka, M., Srisanoh, U., Lartpornmatulee, N., and Boonruangprapa, T. (2007b). ES242 derivatives and cycloheptapeptides from Cordyceps sp. strains BCC 16173 and BCC 16176. J. Nat. Prod. 70, 1601-1604. doi:10.1021/np070357h

Isaka, M., Tanticharoen, M., Kongsaeree, P., and Thebtaranonth, Y. (2001). Structures of cordypyridones A-D, antimalarial N-hydroxy- and $\mathrm{N}$-Methoxy-2-pyridones from the insect pathogenic fungus cordyceps nipponica. J. Org. Chem. 66, 4803-4808. doi:10.1021/jo0100906

Jen, C. Y., Lin, C. Y., Huang, B. M., and Leu, S. F. (2011). Cordycepin induced MA10 mouse Leydig tumor cell apoptosis through caspase-9 pathway. Evid. base Compl. Alternative Med. 2011, 1-11. doi:10.1093/ecam/nen084

Jeong, J. W., Jin, C. Y., Kim, G. Y., Lee, J. D., Park, C., Kim, G. D., et al. (2010). Antiinflammatory effects of cordycepin via suppression of inflammatory mediators in BV2 microglial cells. Int. Immunopharm. 10, 1580-1586. doi:10.1016/j. intimp.2010.09.011

Ji, D. B., Ye, J., Li, C. L., Wang, Y. H., Zhao, J., and Cai, S. Q. (2009). Antiaging effect of Cordyceps sinensis extract. Phytother. Res. 23, 116-122. doi:10. $1002 /$ ptr. 2576 
Ji, J., Liu, J., Liu, H., and Wang, Y. (2014). Effects of fermented mushroom of Cordyceps sinensis, rich in selenium, on uterine cervix cancer. Evid. base Compl. Alternative Med., 1-7. doi:10.1155/2014/173180

Jia, J. M., Tao, H. H., and Feng, B. M. (2009). Cordyceamides A and B from the culture liquid of Cordyceps sinensis (BERK.) SACC. Chem. Pharm. Bull. (Tokyo). 57, 99-101. doi:10.1248/cpb.57.99

Jia, T., and Lau, B. H. S. (1997). The immuno-enhancing effect of Chinese herbal medicine Cordyceps sinensis on macrophage J774. Zhongguo yao xue za zhi. 32, 142-144.

Jiang, F., Liu, C., Li, D., and Jin, B. (2008). Determination of cordycepin from cultured Cordyceps sinensis by HPLC-DAD. Food Sci. 29, 352-354.

Jiang, P. (1987). Pharmacology constituent and function of Cordyceps sinensis. J. Westnorthern Med. 2, 43-44.

Jiang, Y., Wong, J. H., Fu, M., Ng, T. B., Liu, Z. K., Wang, C. R., et al. (2011). Isolation of adenosine, iso-sinensetin and dimethylguanosine with antioxidant and HIV-1 protease inhibiting activities from fruiting bodies of Cordyceps militaris. Phytomedicine. 18, 189-193. doi:10.1016/j.phymed.2010.04.010

Jin, C. Y., Kim, G. Y., and Choi, Y. H. (2008). Induction of apoptosis by aqueous extract of Cordyceps militaris through activation of caspases and inactivation of Akt in human breast cancer MDA-MB-231 Cells. J. Microbiol. Biotechnol. 18, 1997-2003. doi:10.4014/jmb.0800.272

Jin, E., Han, S., Son, M., and Kim, S. W. (2016). Cordyceps bassiana inhibits smooth muscle cell proliferation via the ERK1/2 MAPK signaling pathway. Cell. Mol. Biol. Lett. 21, 24. doi:10.1186/s11658-016-0023-z

Jin, H. L., and Guo, R. X. (2006). Influence of Cordyceps sinensis on reproduction and testis morphology in mice. Shenzhen J. Integrat. Trad. Chin. Western Med. 5, 289-292.

Jing, Y., Cui, X., Chen, Z., Huang, L., Song, L., Liu, T., et al. (2014). Elucidation and biological activities of a new polysaccharide from cultured Cordyceps militaris. Carbohydr. Polym. 102, 288-296. doi:10.1016/j.carbpol.2013.11.061

Jing, Y., Zhu, J., Liu, T., Bi, S., Hu, X., Chen, Z., et al. (2015). Structural characterization and biological activities of a novel polysaccharide from Cultured Cordyceps militaris and its sulfated derivative. J. Agric. Food Chem. 63, 3464-3471. doi:10.1021/jf505915t

Jo, W. S., Choi, Y. J., Kim, H. J., Lee, J. Y., Nam, B. H., Lee, J. D., et al. (2010). The anti-inflammatory effects of water extract from Cordyceps militaris in murine macrophage. Mycobiology. 38, 46. doi:10.4489/MYCO.2010.38.1.046

Jung, S.-J., Jung, E.-S., Choi, E.-K., Sin, H.-S., Ha, K.-C., and Chae, S.-W. (2019). Immunomodulatory effects of a mycelium extract of Cordyceps (Paecilomyces hepiali; CBG-CS-2): a randomized and double-blind clinical trial. BMC Compl. Alternative Med. 19, 77. doi:10.1186/s12906-019-2483-y

Jung, S. H., Lee, Y. S., Lim, S. S., Kim, Y. S., Lee, S., and Shin, K. H. (2009). Hepatoprotective and antioxidant capacities of paecilomyces japonica and Cordyceps sinensis in rats with $\mathrm{CCl}_{4}$-Induced hepatic injury. Korean J. Horticultural Sci. Technol. 27, 668-672.

Kan, W.-C., Wang, H.-Y., Chien, C.-C., Li, S.-L., Chen, Y.-C., Chang, L.-H., et al. (2012). Effects of extract from solid-state fermented Cordyceps sinensison type 2 diabetes mellitus. Evid. base Compl. Alternative Med., 1-10. doi:10.1155/2012/ 743107

Kashyap, D., Tuli, H. S., and Sharma, A. K. (2016). Cordyceps: a natural himalayan Viagra with promising aphrodisiac potential. Austin Andrology. 1, 1010.

Kawagishi, H., Okamura, K., Kobayashi, F., and Kinjo, N. (2004). Estrogenic substances from the mycelia of medicinal fungus Cordyceps ophioglossoides (ehrh.) Fr. (Ascomycetes). Int. J. Med. Mushrooms. 6, 249-252. doi:10.1615/ intjmedmushr.v6.i3.40

Kiho, T., Hui, J., Yamane, A., and Ukai, S. (1993). Polysaccharides in fungi. xxxII. Hypoglycemic activity and chemical properties of a polysaccharide from the cultural mycelium of Cordyceps sinensis. Biol. Pharmaceut. Bull. 16, 1291-1293. doi:10.1248/bpb.16.1291

Kiho, T., Ookubo, K., Usui, S., Ukai, S., and Hirano, K. (1999). Structural features and hypoglycemic activity of a polysaccharide (CS-F10) from the cultured mycelium of Cordyceps sinensis. Biol. Pharmaceut. Bull. 22, 966-970. doi:10. 1248/bpb.22.966

Kiho, T., Yamane, A., Hui, J., Usui, S., and Ukai, S. (1996). Polysaccharides in fungi. xxxVI. Hypoglycemic activity of a polysaccharide (CS-F30) from the cultural mycelium of Cordyceps sinensis and its effect on glucose metabolism in mouse liver. Biol. Pharmaceut. Bull. 19, 294-296. doi:10.1248/bpb.19.294
Kim, D. J., Kang, Y. H., Kim, K. K., Kim, T. W., Park, J. B., and Choe, M. (2017). Increased glucose metabolism and alpha-glucosidase inhibition in Cordyceps militaris water extract-treated HepG2 cells. Nutr. Res. Pract. 11, 180. doi:10. 4162/nrp.2017.11.3.180

Kim, D. Y., Kadam, A., Shinde, S., Saratale, R. G., Patra, J., and Ghodake, G. (2018). Recent developments in nanotechnology transforming the agricultural sector: a transition replete with opportunities. J. Sci. Food Agric. 98, 849-864. doi:10. $1002 /$ jsfa. 8749

Kim, H. G., Shrestha, B., Lim, S. Y., Yoon, D. H., Chang, W. C., Shin, D.-J., et al. (2006). Cordycepin inhibits lipopolysaccharide-induced inflammation by the suppression of NF-kB through Akt and p38 inhibition in RAW 264.7 macrophage cells. Eur. J. Pharmacol. 545, 192-199. doi:10.1016/j.ejphar. 2006.06.047

Kim, H. G., Song, H., Yoon, D. H., Song, B. W., Park, S. M., Sung, G. H., et al. (2010). Cordyceps pruinosa extracts induce apoptosis of HeLa cells by a caspase dependent pathway. J. Ethnopharmacol. 128, 342-351. doi:10.1016/j.jep.2010. 01.049

Kim, H. G., Yang, W. S., Sung, G. H., Kim, J. H., Baek, G. S., Kim, E., et al. (2014). IKK $\beta$-targeted anti-inflammatory activities of a butanol fraction of artificially cultivated Cordyceps pruinosa fruit bodies. Evid. Based Complement Alternat. Med. 2014, 562467. doi:10.1155/2014/562467

Kim, H. S., Kim, J. Y., Kang, J. S., Kim, H. M., Kim, Y. O., Hong, I. P., et al. (2010). Cordlan polysaccharide isolated from mushroom Cordyceps militaris induces dendritic cell maturation through toll-like receptor 4 signalings. Food Chem. Toxicol. 48, 1926-1933. doi:10.1016/j.fct.2010.04.036

Kim, J. H., Lee, Y., Sung, G. H., Kim, H. G., Jeong, D., Park, J. G., et al. (2015). Antiproliferative and apoptosis-inducing activities of 4-Isopropyl-2,6-bis(1phenylethyl)phenol isolated from butanol fraction of Cordyceps bassiana. Evid. Based Complement Alternat. Med. 2015, 739874. doi:10.1155/2015/ 739874

Kim, J. R., Yeon, S. H., Kim, H. S., and Ahn, Y. J. (2002). Larvicidal activity against Plutella xylostella of cordycepin from the fruiting body of Cordyceps militaris. Pest Manag. Sci. 58, 713-717. doi:10.1002/ps.508

Kim, K. M., Kwon, Y. G., Chung, H. T., Yun, Y. G., Pae, H. O., Han, J. A., et al. (2003). Methanol extract of Cordyceps pruinosa inhibits in vitro and in vivo inflammatory mediators by suppressing NF-kappaB activation. Toxicol. Appl. Pharmacol. 190, 1-8. doi:10.1016/s0041-008x(03)00152-2

Kim, M. S., Lee, Y., Sung, G.-H., Kim, J. H., Park, J. G., Kim, H. G., et al. (2015). ProApoptotic activity of 4-isopropyl-2-(1-phenylethyl) aniline isolated from Cordyceps bassiana. Biomol. Therapeu. 23, 367-373. doi:10.4062/biomolther. 2015.021

Kim, T. W., Yoon, D. H., Cho, J. Y., and Sung, G. H. (2014). Anti-inflammatory compounds from Cordyceps bassiana (973.3). Faseb. J. 28, 973. doi:10.1096/ fasebj.28.1_supplement.973.3

Kittakoop, P., Punya, J., Kongsaeree, P., Lertwerawat, Y., Jintasirikul, A., Tanticharoen, M., et al. (1999). Bioactive naphthoquinones from Cordyceps unilateralis. Phytochemistry. 52, 453-457. doi:10.1016/s0031-9422(99)00272-1

Kneifel, H., König, W. A., Loeffler, W., and Müller, R. (1977). Ophiocordin, an antifungal antibiotic of Cordyceps ophioglossoides. Arch. Microbiol. 113, 121-130. doi:10.1007/BF00428591

Koh, J. H., Kim, J. M., Chang, U. J., and Suh, H. J. (2003). Hypocholesterolemic effect of hot-water extract from mycelia of Cordyceps sinensis. Biol. Pharmaceut. Bull. 26, 84-87. doi:10.1248/bpb.26.84

Koh, J. H., Yu, K. W., Suh, H. J., Choi, Y. M., and Ahn, T. S. (2002). Activation of macrophages and the intestinal immune system by an orally administered decoction from cultured mycelia of Cordyceps sinensis. Biosci. Biotechnol. Biochem. 66, 407-411. doi:10.1271/bbb.66.407

Krasnoff, S. B., Reátegui, R. F., Wagenaar, M. M., Gloer, J. B., and Gibson, D. M. (2005). Cicadapeptins I and II: new Aib-containing peptides from the entomopathogenic fungus Cordyceps heteropoda. J. Nat. Prod. 68, 50-55. doi:10.1021/np0497189

Kumar, R., Negi, P. S., Singh, B., Ilavazhagan, G., Bhargava, K., and Sethy, N. K. (2011). Cordyceps sinensis promotes exercise endurance capacity of rats by activating skeletal muscle metabolic regulators. J. Ethnopharmacol. 136, 260-266. doi:10.1016/j.jep.2011.04.040

Kuo, C. F., Chen, C. C., Lin, C. F., Jan, M. S., Huang, R. Y., Luo, Y. H., et al. (2007). Abrogation of streptococcal pyrogenic exotoxin B-mediated suppression of 
phagocytosis in U937 cells by Cordyceps sinensis mycelium via production of cytokines. Food Chem. Toxicol. 45, 278-285. doi:10.1016/j.fct.2006.08.017

Kuo, Y. C., Lin, C. Y., Tsai, W. J., Wu, C. L., Chen, C. F., and Shiao, M. S. (1994). Growth inhibitors against tumor cells in Cordyceps sinensis other than cordycepin and polysaccharides. Canc. Invest. 12, 611-615. doi:10.3109/ 07357909409023046

Kuo, Y. C., Tsai, W. J., Shiao, M. S., Chen, C. F., and Lin, C. Y. (1996). Cordyceps sinensis as an immunomodulatory agent. Am. J. Chin. Med. 24, 111-125. doi:10. $1142 / S 0192415 X 96000165$

Kuo, Y. C., Tsai, W. J., Wang, J. Y., Chang, S. C., Lin, C. Y., and Shiao, M. S. (2001). Regulation of bronchoalveolar lavage fluids cell function by the immunomodulatory agents from Cordyceps sinensis. Life Sci. 68, 1067-1082. doi:10.1016/s0024-3205(00)01011-0

Kuo, Y. C., Weng, S. C., Chou, C. J., Chang, T. T., and Tsai, W. J. (2003). Activation and proliferation signals in primary human $\mathrm{T}$ lymphocytes inhibited by ergosterol peroxide isolated from Cordyceps cicadae. Br. J. Pharmacol. 140, 895-906. doi:10.1038/sj.bjp.0705500

Lee, D., Lee, W. Y., Jung, K., Kwon, Y. S., Kim, D., Hwang, G. S., et al. (2019). The inhibitory effect of Cordycepin on the proliferation of MCF-7 breast cancer cells, and its mechanism: an investigation using network pharmacology-based analysis. Biomolecules. 9, 407. doi:10.3390/biom9090407

Lee, H. H., Kim, S. O., Kim, G. Y., Moon, S. K., Kim, W. J., Jeong, Y. K., et al. (2014). Involvement of autophagy in cordycepin-induced apoptosis in human prostate carcinoma LNCaP cells. Environ. Toxicol. Pharmacol. 38, 239-250. doi:10.1016/ j.etap.2014.06.003

Lee, J. S., and Hong, E. K. (2011). Immunostimulating activity of the polysaccharides isolated from Cordyceps militaris. Int. Immunopharm. 11, 1226-1233. doi:10.1016/j.intimp.2011.04.001

Lee, J. S., Kwon, D. S., Lee, K. R., Park, J. M., Ha, S. J., and Hong, E. K. (2015). Mechanism of macrophage activation induced by polysaccharide from Cordyceps militaris culture broth. Carbohydr. Polym. 120, 29-37. doi:10. 1016/j.carbpol.2014.11.059

Lee, J. S., Kwon, J. S., Yun, J. S., Pahk, J. W., Shin, W. C., Lee, S. Y., et al. (2010). Structural characterization of immunostimulating polysaccharide from cultured mycelia of Cordyceps militaris. Carbohydr. Polym. 80, 1011-1017. doi:10.1016/j.carbpol.2010.01.017

Lee, S. K. W., Kwok Wong, C., Kai Kong, S., Nam Leung, K., and Wai Kei Lam, C. (2006). Immunomodulatory activities of HERBSnSENSES Cordyceps -- in vitro and in vivo studies. Immunopharmacol. Immunotoxicol. 28, 341-360. doi:10. 1080/08923970600809470

Leu, S. F., Chien, C. H., Tseng, C. Y., Kuo, Y. M., and Huang, B. M. (2005). The in Vivo effect of Cordyceps sinensis mycelium on plasma corticosterone level in male mouse. Biol. Pharmaceut. Bull. 28, 1722-1725. doi:10.1248/bpb.28. 1722

Leu, S. F., Poon, S. L., Pao, H. Y., and Huang, B. M. (2011). The in vivo and in vitro stimulatory effects of Cordycepin on mouse Leydig cell steroidogenesis. Biosci. Biotechnol. Biochem. 75, 723-731. doi:10.1271/bbb.100853

Leung, P. H., Zhao, S., Ho, K. P., and Wu, J. Y. (2009). Chemical properties and antioxidant activity of exopolysaccharides from mycelial culture of Cordyceps sinensis fungus Cs-HK1. Food Chem. 114, 1251-1256. doi:10.1016/j.foodchem. 2008.10.081

Li, S., Li, P., and Ji, H. (2011). RP-HPLC determination of ergosterol in natural and cultured Cordyceps. Chin. J. Mod. Appl. Pharm. 18, 297-299.

Li, S. P., Li, P., Dong, T. T., and Tsim, K. W. (2001a). Anti-oxidation activity of different types of natural Cordyceps sinensis and cultured Cordyceps mycelia. Phytomedicine. 8, 207-212. doi:10.1078/0944-7113-00030

Li, S. P., Li, P., Dong, T. T., and Tsim, K. W. (2001b). Determination of nucleosides in natural Cordyceps sinensis and cultured Cordyceps mycelia by capillary electrophoresis. Electrophoresis. 22, 144-150. doi:10.1002/1522-2683(200101) 22:1<144::AID-ELPS144>3.0.CO;2-T

Li, S. P., Li, P., Ji, H., Zhang, P., Dong, T. T., and Tsim, K. W. (2001c). [The contents and their change of nucleosides from natural Cordyceps sinensis and cultured Cordyceps mycelia]. Yao Xue Xue Bao. 36, 436-439.

Li, S. P., Li, P., Lai, C. M., Gong, Y. X., Kan, K. K., Dong, T. T., et al. (2004). Simultaneous determination of ergosterol, nucleosides and their bases from natural and cultured Cordyceps by pressurised liquid extraction and highperformance liquid chromatography. J. Chromatogr. A. 1036, 239-243. doi:10. 1016/j.chroma.2004.02.080
Li, S. P., and Wang, Y. T. (2008). Pharmacological activity-based quality control of Chinese herbs. New York, NY: Nova Science Publishers.

$\mathrm{Li}, \mathrm{T}$., and Li, W. (2009). Impact of polysaccharides from Cordyceps on anti-fatigue in mice. Sci. Res. Essays. 4, 705-709. doi:10.5897/SRE.9000570

Li, X. G., Pan, W. D., Lou, H. Y., Liu, R. M., Xiao, J. H., and Zhong, J. J. (2015). New cytochalasins from medicinal macrofungus Crodyceps taii and their inhibitory activities against human cancer cells. Bioorg. Med. Chem. Lett. 25, 1823-1826. doi:10.1016/j.bmcl.2015.03.059

Li, Y. H., and Li, X. L. (1991). [Determination of ergosterol in Cordyceps sinensis and Cordyceps black-bone chicken capsules by HPLC]. Yao Xue Xue Bao. 26, 768-771.

Li, Y., Xue, W. J., Tian, P. X., Ding, X. M., Yan, H., Pan, X. M., et al. (2009). Clinical application of Cordyceps sinensis on immunosuppressive Therapy in renal transplantation. Transplant. Proc. 41, 1565-1569.

Lim, H. W., Kwon, Y. M., Cho, S. M., Kim, J. H., Yoon, G. H., Lee, S., et al. (2004). Antitumor activity of Cordyceps militaris on human cancer cell line. Korean J. Pharmacogn. 35, 364-367.

Lin, B.-Q., and Li, S.-P. (2011). Cordyceps as an herbal drug. Boca Raton, FL: CRC Press.

Lin, W.-H., Tsai, M.-T., Chen, Y.-S., Hou, R. C.-W., Hung, H.-F., Li, C.-H., et al. (2007). Improvement of sperm production in subfertile boars by Cordyceps militaris supplement. Am. J. Chin. Med. 35, 631-641. doi:10.1142/ S0192415X07005120

Liu, C.-Q., Zheng, A.-J., Li, D.-J., and Cao, Q.-S. (2007). Improvement of immunity in mice by ultra-fine pulverization of Cordyceps militaris. Jiangsu J. Agric. Sci. 1, 63-66.

Liu, J., and Fei, Y. (2001). Enhancement of Cordyceps taii polysaccharide and Cordyceps pruinosa polysaccharide on cellular immune function in vitro. J. Immun. 17, 189-191.

Liu, J. Y., Feng, C. P., Li, X., Chang, M. C., Meng, J. I., and Xu, L. J. (2016). Immunomodulatory and antioxidative activity of Cordyceps militaris polysaccharides in mice. Int. J. Biol. Macromol. 86, 594-598. doi:10.1016/j. ijbiomac.2016.02.009

Liu, R. M., Zhang, X. J., Liang, G. Y., Yang, Y. F., Zhong, J. J., and Xiao, J. H. (2015). Antitumor and antimetastatic activities of chloroform extract of medicinal mushroom Cordyceps taii in mouse models. BMC Compl. Alternative Med. 15, 216. doi:10.1186/s12906-015-0797-y

Liu, Y., Wang, J., Wang, W., Zhang, H., Zhang, X., and Han, C. (2015). The chemical constituents and pharmacological actions of Cordyceps sinensis. Evid Based Complement Alternat. Med. 2015, 575063. doi:10.1155/2015/575063

Lo, H.-C., Hsieh, C., Lin, F.-Y., and Hsu, T.-H. (2013). A systematic review of the mysterious caterpillar fungus ophiocordyceps sinensis in DongChongXiaCao (冬蟲夏草 dōng chóng xià căo) and related bioactive ingredients. J. Tradit. Complement Med. 3, 16-32. doi:10.4103/2225-4110.106538

Lo, H.-C., Tu, S.-T., Lin, K.-C., and Lin, S.-C. (2004). The anti-hyperglycemic activity of the fruiting body of Cordyceps in diabetic rats induced by nicotinamide and streptozotocin. Life Sci. 74, 2897-2908. doi:10.1016/j.lfs. 2003.11.003

Lu, J., Gu, G., Hao, L., Jin, Z., and Wang, X. (2016). Characterization and in vitro antioxidant activity of a polysaccharide from Cordyceps sobolifera. J. Food Process. Preserv. 40, 447-452. doi:10.1111/jfpp.12622

Lu, M. Y., Chen, C. C., Lee, L. Y., Lin, T. W., and Kuo, C. F. (2015). N(6)-(2Hydroxyethyl)adenosine in the medicinal mushroom Cordyceps cicadae attenuates lipopolysaccharide-stimulated pro-inflammatory responses by suppressing TLR4-mediated NF-kB signaling pathways. J. Nat. Prod. 78, 2452-2460. doi:10.1021/acs.jnatprod.5b00573

Lu, R. L., Bao, G. H., Hu, F. L., Huang, B., Li, C. R., and Li, Z. Z. (2014). Comparison of cytotoxic extracts from fruiting bodies, infected insects and cultured mycelia of Cordyceps formosana. Food Chem. 145, 1066-1071. doi:10.1016/j.foodchem. 2013.09.001

Lu, R. L., Luo, F. F., Hu, F. L., Huang, B., Li, C. R., and Bao, G. H. (2013). Identification and production of a novel natural pigment, cordycepoid $\mathrm{A}$, from Cordyceps bifusispora. Appl. Microbiol. Biotechnol. 97, 6241-6249. doi:10.1007/ s00253-013-4966-y

Lü, Y.-H., Pan, W.-D., Xiao, J.-H., Sun, Z.-H., and Zhong, J.-J. (2014). Cytotoxic mechanism of novel compound jiangxienone from Cordyceps jiangxiensis against cancer cells involving DNA damage response pathway. Process Biochem. 49, 697-705. doi:10.1016/j.procbio.2014.01.027 
Ma, L., Zhang, S., and Du, M. (2015). Cordycepin from Cordyceps militaris prevents hyperglycemia in alloxan-induced diabetic mice. Nutr. Res. 35, 431-439. doi:10.1016/j.nutres.2015.04.011

Ma, M.-W., Gao, X.-S., Yu, H.-L., Qi, X., Sun, S.-Q., and Wang, D. (2018). Cordyceps sinensis promotes the growth of prostate cancer cells. Nutr. Canc. 70, 1166-1172. doi:10.1080/01635581.2018.1504091

Maity, D. (2013). A study on ethnomedicinal uses of yartshagumba, Cordyceps sinensis (berk.) sacc. (cordycipitaceae), by the trinal communities of North Sikkim and its conservation. Explor. Anim. Med. Res. 3, 95-101.

Matsuda, H., Akaki, J., Nakamura, S., Okazaki, Y., Kojima, H., Tamesada, M., et al. (2009). Apoptosis-inducing effects of sterols from the dried powder of cultured mycelium of Cordyceps sinensis. Chem. Pharm. Bull. 57, 411-414. doi:10.1248/ cpb.57.411

Meng, Z., Kang, J., Wen, T., Lei, B., and Hyde, K. D. (2015). Cordycepin and N6-(2hydroxyethyl)-adenosine from Cordyceps pruinosa and their interaction with human serum albumin. PloS One. 10, e0121669. doi:10.1371/journal.pone. 0121669

Mishra, R. N., and Upadhyay, Y. (2011). Cordicepssinensis: the Chinese Rasayancurrent research scenario. Int. J. Res. Pharm. Biomed. Sci. 2, 1503-1519. doi:10. 4103/2231-4040.90873

Mizuno, T. (1999). Medicinal effects and utilization of cordyceps (Fr.) link (ascomycetes) and Isaria Fr. (mitosporic fungi) Chinese caterpillar fungi, "Tochukaso" (review). Int. J. Med. Mushrooms. 1, 251-261. doi:10.1615/ intjmedmushrooms.v1.i3.80

Nakamura, K., Shinozuka, K., and Yoshikawa, N. (2015). Anticancer and antimetastatic effects of cordycepin, an active component of Cordyceps sinensis. J. Pharmacol. Sci. 127, 53-56. doi:10.1016/j.jphs.2014.09.001

Nakamura, K., Yamaguchi, Y., Kagota, S., Shinozuka, K., and Kunitomo, M. (1999). Activation of in vivo kupffer cell function by oral administration of Cordyceps sinensis in rats. Jpn. J. Pharmacol. 79, 505-508. doi:10.1254/jjp. 79.505

Nallathamby, N., Guan-Serm, L., Vidyadaran, S., Abd Malek, S. N., Raman, J., and Sabaratnam, V. (2015). Ergosterol of Cordyceps militaris attenuates LPS induced inflammation in BV2 microglia cells. Nat. Prod. Commun. 10, 885-886. doi:10.1177/1934578x1501000623

Nishizawa, K., Torii, K., Kawasaki, A., Katada, M., Ito, M., Terashita, K., et al. (2007). Antidepressant-like effect of Cordyceps sinensis in the mouse tail suspension test. Biol. Pharmaceut. Bull. 30, 1758-1762. doi:10.1248/bpb.30. 1758

Nomani, A. Z., Nabi, Z., Rashid, H., Janjua, J., Nomani, H., Majeed, A., et al. (2014). Osmotic nephrosis with mannitol: review article. Ren. Fail. 36, 1169-1176. doi:10.3109/0886022X.2014.926758

Oh, J. Y., Baek, Y. M., Kim, S. W., Hwang, H. J., Hwang, H. S., Lee, S. H., et al. (2008). Apoptosis of human hepatocarcinoma (HepG2) and neuroblastoma (SKN-SH) cells induced by polysaccharides-peptide complexes produced by submerged mycelial culture of an entomopathogenic fungus Cordyceps sphecocephala. J. Microbiol. Biotechnol. 18, 512-519.

Ohta, Y., Lee, J.-B., Hayashi, K., Fujita, A., Park, D. K., and Hayashi, T. (2007). In Vivo anti-influenza virus activity of an immunomodulatory acidic polysaccharide isolated from Cordyceps militaris grown on germinated soybeans. J. Agric. Food Chem. 55, 10194-10199. doi:10.1021/jf0721287

Olatunji, O. J., Feng, Y., Olatunji, O. O., Tang, J., Wei, Y., Ouyang, Z., et al. (2016). Polysaccharides purified from Cordyceps cicadae protects PC12 cells against glutamate-induced oxidative damage. Carbohydr. Polym. 153, 187-195. doi:10. 1016/j.carbpol.2016.06.108

Olatunji, O. J., Tang, J., Tola, A., Auberon, F., Oluwaniyi, O., and Ouyang, Z. (2018). The genus Cordyceps: an extensive review of its traditional uses, phytochemistry and pharmacology. Fitoterapia. 129, 293-316. doi:10.1016/j. fitote.2018.05.010

Pal, M., and Misra, K. (2018). "Cordyceps sp.: the precious mushroom for highaltitude maladies," in Management of high altitude pathophysiology. Editors K. Misra, P. Sharma, and A. Bhardwaj (Cambridge, MA: Academic Press), 93-114

Pan, B. S., Lin, C. Y., and Huang, B. M. (2011). The effect of Cordycepin on steroidogenesis and apoptosis in MA-10 mouse Leydig tumor cells. Evid. base Compl. Alternative Med. 2011, 750468. doi:10.1155/2011/750468

Panda, A. K. (2010). Tracing historical perspective of Cordyceps sinensis -an aphrodisiac in Sikkim Himalaya. Indian J. Hist. Sci. 2, 189-198.
Panda, A. K., and Swain, K. C. (2011). Traditional uses and medicinal potential of Cordyceps sinensis of Sikkim. J. Ayurveda Integr. Med. 2, 9-13. doi:10.4103/ 0975-9476.78183

Pao, H. Y., Pan, B. S., Leu, S. F., and Huang, B. M. (2012). Cordycepin stimulated steroidogenesis in MA-10 mouse Leydig tumor cells through the protein kinase C pathway. J. Agric. Food Chem. 60, 4905-4913. doi:10.1021/jf205091b

Park, C., Hong, S., Lee, J.-Y., Kim, G.-Y., Choi, B., Lee, Y., et al. (2005). Growth inhibition of U937 leukemia cells by aqueous extract of Cordyceps militaris through induction of apoptosis. Oncol. Rep. 13, 1211-1216. doi:10.3892/or.13.6. 1211

Park, J. G., Son, Y. J., Lee, T. H., Baek, N. J., Yoon, D. H., Kim, T. W., et al. (2017). Anticancer efficacy of Cordyceps militaris ethanol extract in a xenografted leukemia model. Evid Based Complement Alternat. Med. 2017, 8474703. doi:10. $1155 / 2017 / 8474703$

Park, S. Y., Jung, S. J., Ha, K. C., Sin, H. S., Jang, S. W., Chae, H. J., et al. (2014). Anti-inflammatory effects of Cordyceps mycelium (Paecilomyces hepiali, CBGCS-2) in Raw264.7 murine macrophages. Orient. Pharm. Exp. Med. 15, 7-12. doi:10.1007/s13596-014-0173-3

Paterson, R. R. (2008). Cordyceps: a traditional Chinese medicine and another fungal therapeutic biofactory?. Phytochemistry. 69, 1469-1495. doi:10.1016/j. phytochem.2008.01.027

Pereira, C. G., and Meireles, M. A. A. (2010). Supercritical fluid extraction of bioactive compounds: fundamentals, applications and economic perspectives. Food Bioprocess Technol. 3, 340-372. doi:10.1007/s11947-009-0263-2

Qian, G. M., Pan, G. F., and Guo, J. Y. (2012). Anti-inflammatory and antinociceptive effects of cordymin, a peptide purified from the medicinal mushroom Cordyceps sinensis. Nat. Prod. Res. 26, 2358-2362. doi:10.1080/ 14786419.2012.658800

Rao, Y. K., Fang, S. H., and Tzeng, Y. M. (2007). Evaluation of the antiinflammatory and anti-proliferation tumoral cells activities of Antrodia camphorata, Cordyceps sinensis, and Cinnamomum osmophloeum bark extracts. J. Ethnopharmacol. 114, 78-85. doi:10.1016/j.jep.2007.07.028

Rao, Y. K., Fang, S. H., Wu, W. S., and Tzeng, Y. M. (2010). Constituents isolated from Cordyceps militaris suppress enhanced inflammatory mediator's production and human cancer cell proliferation. J. Ethnopharmacol. 131, 363-367. doi:10.1016/j.jep.2010.07.020

Reis, F. S., Barros, L., Calhelha, R. C., Cirić, A., Van Griensven, L. J., Soković, M., et al. (2013). The methanolic extract of Cordyceps militaris (L.) Link fruiting body shows antioxidant, antibacterial, antifungal and antihuman tumor cell lines properties. Food Chem. Toxicol. 62, 91-98. doi:10.1016/j.fct.2013. 08.033

Rhee, K. H. (2004). Cyclic dipeptides exhibit synergistic, broad spectrum antimicrobial effects and have anti-mutagenic properties. Int. J. Antimicrob. Agents. 24, 423-427. doi:10.1016/j.ijantimicag.2004.05.005

Romano, A. D., Serviddio, G., De Matthaeis, A., Bellanti, F., and Vendemiale, G. (2010). Oxidative stress and aging. J. Nephrol. 23 (Suppl. 15), S29-S36.

Seephonkai, P., Isaka, M., Kittakoop, P., Trakulnaleamsai, S., Rattanajak, R., Tanticharoen, M., et al. (2001). A new tropolone from the insect pathogenic fungus Cordyceps sp. BCC 1681. J. Antibiot. (Tokyo). 54, 751-752. doi:10.7164/ antibiotics.54.751

Segelken, R. (1996). Cyclosporin mold's "sexual state"found in New York forest Cornell students' discovery could target additional sources of nature-based pharmaceuticals. Available at: https://news.cornell.edu/stories/1996/09/ cyclosporin-molds-sexual-state-found-new-york-forest-cornell-studentsdiscovery.

Shaoping, L., Ping, L., Hui, J., Quan, Z., Dong, T. T., and Tsim, K. W. (2001). The nucleosides contents and their variation in natural Cordyceps sinensis and cultured Cordyceps mycelia. J. Chin. Pharmaceut. Sci. 10, 175-179.

Shashidhar, M. G., Giridhar, P., Udaya Sankar, K., and Manohar, B. (2013). Bioactive principles from Cordyceps sinensis: a potent food supplement - a review. J. Funct. Foods. 5, 1013-1030. doi:10.1016/j.jff.2013.04.018

Sheng, L., Chen, J., Li, J., and Zhang, W. (2011). An exopolysaccharide from cultivated Cordyceps sinensis and its effects on cytokine expressions of immunocytes. Appl. Biochem. Biotechnol. 163, 669-678. doi:10.1007/s12010010-9072-3

Shiao, M. S., Wang, Z. N., Lin, L. J., Lien, J. Y., and Wang, J. J. (1994). Profiles of nucleosides and nitrogen bases in Chinese medicinal fungus Cordyceps sinensis and related species. Bot. Bull. Acad. Sin. 35, 261. 
Shim, J., Lee, Y., Lim, S., Shin, K., Hyun, J., Kim, S., et al. (2000). Pharmacological activities of Paecilomyces japonica, a new type Cordyceps sp. Korean J. Pharmacogn. 31, 163-167.

Shin, K. H., Lim, S. S., Lee, S. H., Lee, Y. S., and Cho, S. Y. (2001). Antioxidant and immunostimulating activities of the fruiting bodies of Paecilomyces japonica, a new type of Cordyceps sp. Ann. N. Y. Acad. Sci. 928, 261-273. doi:10.1111/j. 1749-6632.2001.tb05655.x

Shin, K. H., Lim, S. S., Lee, S. H., Lee, Y. S., Jung, S. H., and Cho, S. Y. (2003). Antitumour and immuno-stimulating activities of the fruiting bodies of Paecilomyces japonica, a new type of Cordyceps spp. Phytother Res. 17, 830-833. doi:10.1002/ptr.1253

Shunzhi, C., and Jingzhi, C. (1996). NMR and IR studies on the characterization of cordycepin and 2'-deoxyadenosine. Chin. J. Natibiotics. 1.

Singh, K. P., Meena, H. S., and Negi, P. S. (2014). Enhancement of neuromuscular activity by natural specimens and cultured mycelia of Cordyceps sinensis in mice. Indian J. Pharmaceut. Sci. 76, 458-461. doi:10.4103/0250-474X.143105

Sohn, S.-H., Lee, S.-C., Hwang, S.-Y., Kim, S.-W., Kim, I.-W., Ye, M., et al. (2012). Effect of long-term administration of cordycepin from Cordyceps militaris on testicular function in middle-aged rats. Planta Med. 78, 1620-1625. doi:10. 1055/s-0032-1315212

Song, J., Wang, H., Sun, M., Wang, K., Xu, F., Shao, X., et al. (2018). Optimization of extraction and antioxidant activity of polysaccharides from Cordyceps cicadae by response sufrace methodology. Food Sci. 39, 275-281.

Song, J., Wang, Y., Teng, M., Cai, G., Xu, H., Guo, H., et al. (2015). Studies on the antifatigue activities of Cordyceps militaris Fruit body extract in mouse model. Evid. base Compl. Alternative Med., 1-15. doi:10.1155/2015/174616

Sprecher, M., and Sprinson, D. B. (1963). A reinvestigation of the structure of "cordycepic Acid"1a. J. Org. Chem. 28, 2490-2491. doi:10.1021/jo01044a536

Suh, W., Nam, G., Yang, W. S., Sung, G.-H., Shim, S. H., and Cho, J. Y. (2017). Chemical constituents identified from fruit body of Cordyceps bassiana and their anti-inflammatory activity. Biomol. Therapeutics. 25, 165-170. doi:10. 4062/biomolther.2016.063

Sun, H., Zhu, Z., Tang, Y., Ren, Y., Song, Q., Tang, Y., et al. (2018). Structural characterization and antitumor activity of a novel Se-polysaccharide from selenium-enriched Cordyceps gunnii. Food Funct. 9, 2744-2754. doi:10.1039/ c8fo00027a

Sun, J., Xu, J., Wang, S., Hou, Z., Lu, X., An, L., et al. (2019). A new cerebroside from Cordyceps militaris with anti-PTP1B activity. Fitoterapia. 138, 104342. doi:10. 1016/j.fitote.2019.104342

Sun, Y., Zhang, X., and Lei, P. (2003). Study on character for separation and extraction of polysaccharide from hyphae of Cordyceps sinersis. Acta Chin. Med. Pharmacol. 2, 3.

Sun, Y., Zhao, Z., Feng, Q., Xu, Q., Lü, L., Liu, J.-K., et al. (2013). Unusual spirodecane sesquiterpenes and a fumagillol analogue from Cordyceps ophioglossoides. Helv. Chim. Acta. 96, 76-84. doi:10.1002/hlca.201200068

Tuli, H. S., Sandhu, S. S., and Sharma, A. K. (2013a). Pharmacological and therapeutic potential of Cordyceps with special reference to Cordycepin. Biotechnology. 3, 1-12. doi:10.1007/s13205-013-0121-9

Tuli, H. S., Sharma, A. K., Sandhu, S. S., and Kashyap, D. (2013b). Cordycepin: a bioactive metabolite with therapeutic potential. Life Sci. 93, 863-869. doi:10. 1016/j.lfs.2013.09.030

Ukai, S., Kiho, T., Hara, C., Morita, M., Goto, A., Imaizumi, N., et al. (1983). Polysaccharides in fungi. XIII. Antitumor activity of various polysaccharides isolated from Dictyophora indusiata, Ganoderma japonicum, Cordyceps cicadae, Auricularia auricula-judae, and Auricularia species. Chem. Pharm. Bull. (Tokyo). 31, 741-744. doi:10.1248/cpb.31.741

Umeyama, A., Takahashi, K., Grudniewska, A., Shimizu, M., Hayashi, S., Kato, M., et al. (2014). In vitro antitrypanosomal activity of the cyclodepsipeptides, cardinalisamides A-C, from the insect pathogenic fungus Cordyceps cardinalis NBRC 103832. J. Antibiot. 67, 163-166. doi:10.1038/ja.2013.93

Varughese, T., Riosa, N., Higginbotham, S., Arnold, A. E., Coley, P. D., Kursar, T. A., et al. (2012). Antifungal depsidone metabolites from Cordyceps dipterigena, an endophytic fungus antagonistic to the phytopathogen Gibberella fujikuroi. Tetrahedron Lett. 53, 1624-1626. doi:10.1016/j.tetlet.2012.01.076

Wang, B. J., Won, S. J., Yu, Z. R., and Su, C. L. (2005). Free radical scavenging and apoptotic effects of Cordyceps sinensis fractionated by supercritical carbon dioxide. Food Chem. Toxicol. 43, 543-552. doi:10.1016/j.fct. 2004.12.008
Wang, D., Wang, J., Wang, D., Yu, X., Olatunji, O. J., Ouyang, Z., et al. (2018). Neuroprotective effects of butanol fraction of Cordyceps cicadae on glutamateinduced damage in PC12 cells involving oxidative toxicity. Chem. Biodivers. 15, 385. doi:10.1002/cbdv.201700385

Wang, H., Zhang, J., Sit, W. H., Lee, C. Y., and Wan, J. M. (2014). Cordyceps cicadae induces $\mathrm{G} 2 / \mathrm{M}$ cell cycle arrest in MHCC $97 \mathrm{H}$ human hepatocellular carcinoma cells: a proteomic study. Chin. Med. 9, 15. doi:10.1186/17498546-9-15

Wang, J., Chen, C., Jiang, Z., Wang, M., Jiang, H., and Zhang, X. (2016). Protective effect of Cordyceps militaris extract against bisphenol A induced reproductive damage. Syst. Biol. Reprod. Med. 62, 249-257. doi:10.1080/19396368.2016. 1182234

Wang, J., Zhang, D.-M., Jia, J.-F., Peng, Q.-L., Tian, H.-Y., Wang, L., et al. (2014). Cyclodepsipeptides from the ascocarps and insect-body portions of fungus Cordyceps cicadae. Fitoterapia. 97, 23-27. doi:10.1016/j.fitote.2014.05.010

Wang, L., Xu, N., Zhang, J., Zhao, H., Lin, L., Jia, S., et al. (2015). Antihyperlipidemic and hepatoprotective activities of residue polysaccharide from Cordyceps militaris SU-12. Carbohydr. Polym. 131, 355-362. doi:10.1016/ j.carbpol.2015.06.016

Wang, M., Meng, X. Y., Yang, R. L., Qin, T., Wang, X. Y., Zhang, K. Y., et al. (2012). Cordyceps militaris polysaccharides can enhance the immunity and antioxidation activity in immunosuppressed mice. Carbohydr. Polym. 89, 461-466. doi:10.1016/j.carbpol.2012.03.029

Wang, S. M., Lee, L. J., Lin, W. W., and Chang, C. M. (1998). Effects of a watersoluble extract of Cordyceps sinensis on steroidogenesis and capsular morphology of lipid droplets in cultured rat adrenocortical cells. J. Cell. Biochem. 69, 483-489. doi:10.1002/(sici)1097-4644(19980615)69:4<483::aidjcb9>3.0.co;2-j

Wang, S. X., Liu, Y., Zhang, G. Q., Zhao, S., Xu, F., Geng, X. L., et al. (2012). Cordysobin, a novel alkaline serine protease with HIV-1 reverse transcriptase inhibitory activity from the medicinal mushroom Cordyceps sobolifera. J. Biosci. Bioeng. 113, 42-47. doi:10.1016/j.jbiosc.2011.09.005

Wang, Y. H., Ye, J., Li, C. L., Cai, S. Q., Ishizaki, M., and Katada, M. (2004). An experimental study on anti-aging action of Cordyceps extract. Zhongguo Zhongyao Zazhi. 29, 773-776.

Wang, Y. W., Hong, T. W., Tai, Y. L., Wang, Y. J., Tsai, S. H., Lien, P. T., et al. (2015). Evaluation of an epitypified ophiocordyceps formosana (cordyceps s.l.) for its pharmacological potential. Evid Based Complement Alternat. Med. 2015, 189891. doi:10.1155/2015/189891

Wang, Z.-M., Peng, X., Lee, K.-L. D., Tang, J. C.-O., Cheung, P. C.-K., and Wu, J.-Y. (2011). Structural characterisation and immunomodulatory property of an acidic polysaccharide from mycelial culture of Cordyceps sinensis fungus CsHK1. Food Chem. 125, 637-643. doi:10.1016/j.foodchem.2010.09.052

Wasser, S. P. (2002). Medicinal mushrooms as a source of antitumor and immunomodulating polysaccharides. Appl. Microbiol. Biotechnol. 60, 258-274. doi:10.1007/s00253-002-1076-7

Wei, P.-Y., Liu, L.-X., Liu, T., Chen, C., Luo, D.-Q., and Shi, B.-Z. (2015). Three new pigment protein tyrosine phosphatases inhibitors from the insect parasite fungus Cordyceps gracilioides: terreusinone A, pinophilin $\mathrm{C}$ and cryptosporioptide A. Molecules. 20, 5825-5834. doi:10.3390/ molecules 20045825

Weng, S. C., Chou, C. J., Lin, L. C., Tsai, W. J., and Kuo, Y. C. (2002). Immunomodulatory functions of extracts from the Chinese medicinal fungus Cordyceps cicadae. J. Ethnopharmacol. 83, 79-85. doi:10.1016/s03788741(02)00212-x

Won, K. J., Lee, S. C., Lee, C. K., Lee, H. M., Lee, S. H., Fang, Z., et al. (2009). Cordycepin attenuates neointimal formation by inhibiting reactive oxygen species-mediated responses in vascular smooth muscle cells in rats. J. Pharmacol. Sci. 109, 403-412. doi:10.1254/jphs.08308fp

Won, S. Y., and Park, E. H. (2005). Anti-inflammatory and related pharmacological activities of cultured mycelia and fruiting bodies of Cordyceps militaris. J. Ethnopharmacol. 96, 555-561. doi:10.1016/j.jep.2004.10.009

Wong, K. L., So, E. C., Chen, C. C., Wu, R. S., and Huang, B. M. (2007). Regulation of steroidogenesis by Cordyceps sinensis mycelium extracted fractions with (hCG) treatment in mouse Leydig cells. Arch. Androl. 53, 75-77. doi:10.1080/ 01485010600915236

Wong, Y. Y., Moon, A., Duffin, R., Barthet-Barateig, A., Meijer, H. A., Clemens, M. J., et al. (2010). Cordycepin inhibits protein synthesis and cell adhesion through 
effects on signal transduction. J. Biol. Chem. 285, 2610-2621. doi:10.1074/jbc. M109.071159

Wu, F. Y., Yan, H., Ma, X. N., Jia, J. Q., Zhang, G. Z., Guo, X. J., et al. (2011). Structural characterization and antioxidant activity of purified polysaccharide from cultured Cordyceps militaris. Afr. J. Microbiol. Res. 5, 2743-2751. doi:10. 5897/AJMR11.548

Wu, G., Li, L., Sung, G. H., Kim, T. W., Byeon, S. E., Cho, J. Y., et al. (2011). Inhibition of 2,4-dinitrofluorobenzene-induced atopic dermatitis by topical application of the butanol extract of Cordyceps bassiana in NC/Nga mice. J. Ethnopharmacol. 134, 504-509. doi:10.1016/j.jep.2010.12.012

Wu, J. Y., Zhang, Q. X., and Leung, P. H. (2007). Inhibitory effects of ethyl acetate extract of Cordyceps sinensis mycelium on various cancer cells in culture and B16 melanoma in C57BL/6 mice. Phytomedicine. 14, 43-49. doi:10.1016/j. phymed.2005.11.005

Wu, M. F., Li, P. C., Chen, C. C., Ye, S. S., Chien, C. T., and Yu, C. C. (2011). Cordyceps sobolifera extract ameliorates lipopolysaccharide-induced renal dysfunction in the rat. Am. J. Chin. Med. 39, 523-535. doi:10.1142/ S0192415X11009007

Wu, P., Tao, Z., Liu, H., Jiang, G., Ma, C., Wang, C., et al. (2015). Effects of heat on the biological activity of wild Cordyceps sinensis. J. Trad. Chin. Med. Sci. 2, 32-38. doi:10.1016/j.jtcms.2014.12.005

Wu, W. D., Hu, Z. M., Shang, M. J., Zhao, D. J., Zhang, C. W., Hong, D. F., et al. (2014). Cordycepin down-regulates multiple drug resistant (MDR)/HIF-1 $\alpha$ through regulating AMPK/mTORC1 signaling in GBC-SD gallbladder cancer cells. Int. J. Mol. Sci. 15, 12778-12790. doi:10.3390/ijms150712778

Wu, Y., Sun, H., Qin, F., Pan, Y., and Sun, C. (2006). Effect of various extracts and a polysaccharide from the edible mycelia of Cordyceps sinensis on cellular and humoral immune response against ovalbumin in mice. Phytother. Res. 20, 646-652. doi:10.1002/ptr.1921

Xiao, G., Miyazato, A., Abe, Y., Zhang, T., Nakamura, K., Inden, K., et al. (2010). Activation of myeloid dendritic cells by deoxynucleic acids from Cordyceps sinensis via a Toll-like receptor 9-dependent pathway. Cell. Immunol. 263, 241-250. doi:10.1016/j.cellimm.2010.04.006

Xiao, J., Jiang, N., Liang, Z., and Liu, A. (2003). Research and application prospect of polysaccharides of Cordyceps sinensis. J. Moun. Agric. Biol. 22, 70-76.

Xiao, J.-H., Chen, D.-X., Fang, N., Liu, Z.-L., and Zhang, T. (2006). Growth arrest of human gastric adenocarcinoma cells by bioactive compounds of Cordyceps jiangxiensis (CaoMuWang) through induction of apoptosis. J. Food Agric. Environ. 4, 66-73. doi:10.1234/4.2006.919

Xiao, J.-H., Liang, Z.-Q., Liu, A.-Y., Chen, D.-X., Xiao, Y., Liu, J.-W., et al. (2004a). Immunosuppressive activity of polysaccharides from Cordyceps gunnii mycelia in mice in vivo/vitro. J. Food Agric. Environ. 2, 69-73. doi:10.1234/4.2004.257

Xiao, J.-H, Liang, Z., Liu, A., Wan, W., Xiao, Y., and Liu, J. (2004b). [The effect of ultrasonication on immuno-modulating activity of mycelial polysaccharide from Cordyceps gunnii]. Zhong Yao Cai. 27, 747-750.

Xiao, J.-H., Xiao, D., Sun, Z., Xiao, Y., and Zhong, J.-J. (2011). Antioxidative potential of polysaccharide fractions produced from traditional Chinese medicinal macrofungus Cordyceps jiangxiensis in vitro. Afr. J. Biotechnol. 10, 6607-6615. doi:10.5897/AJB10.2679

Xiao, J. H., Liang, Z. Q., and Liu, A. Y. (2002). [Advances of polysaccharides research and exploitation of anamorph and its related fungi from Cordyceps]. Yao Xue Xue Bao. 37, 589-592.

Xiao, J. H., Sun, Z. H., Pan, W. D., Lü, Y. H., Chen, D. X., and Zhong, J. J. (2012a). Jiangxienone, a new compound with potent cytotoxicity against tumor cells from traditional Chinese medicinal mushroom Cordyceps jiangxiensis. Chem. Biodivers. 9, 1349-1355. doi:10.1002/cbdv.201100244

Xiao, J. H., Xiao, D. M., Chen, D. X., Xiao, Y., Liang, Z. Q., and Zhong, J. J. (2012b). Polysaccharides from the medicinal mushroom Cordyceps taii show antioxidant and immunoenhancing activities in a D-galactose-induced aging mouse model. Evid Based Complement Alternat. Med. 2012, 273435. doi:10.1155/2012/273435

Xiao, Y., Huang, X. Z., Chen, G., Wang, M. B., Zhu, J. S., and Cooper, C. B. (1999). Increased aerobic capacity in healthy elderly humans given a fermentation product of Cordyceps Cs-4. Med. Sci. Sports Exerc. 31, S174. doi:10.1097/ 00005768-199905001-00773

Xie, H., Li, X., Chen, Y., Lang, M., Shen, Z., and Shi, L. (2019). Ethanolic extract of Cordyceps cicadae exerts antitumor effect on human gastric cancer SGC-7901 cells by inducing apoptosis, cell cycle arrest and endoplasmic reticulum stress. J. Ethnopharmacol. 231, 230-240. doi:10.1016/j.jep.2018.11.028
Xu, R. H., Peng, X. E., Chen, G. Z., and Chen, G. L. (1992). Effects of Cordyceps sinensis on natural killer activity and colony formation of B16 melanoma. Chin. Med. J. 105, 97-101.

Yamaguchi, Y., Kagota, S., Nakamura, K., Shinozuka, K., and Kunitomo, M. (2000a). Antioxidant activity of the extracts from fruiting bodies of cultured Cordyceps sinensis. Phytother Res. 14, 647-649. doi:10.1002/1099-1573(200012) 14:8<647::aid-ptr670>3.0.co;2-w

Yamaguchi, Y., Kagota, S., Nakamura, K., Shinozuka, K., and Kunitomo, M. (2000b). Inhibitory effects of water extracts from fruiting bodies of cultured Cordyceps sinensis on raised serum lipid peroxide levels and aortic cholesterol deposition in atherosclerotic mice. Phytother Res. 14, 650-652. doi:10.1002/ 1099-1573(200012)14:8<650::aid-ptr675>3.0.co;2-0

Yan, F., Zhang, Y., and Wang, B. (2012). Effects of polysaccharides from Cordyceps sinensis mycelium on physical fatigue in mice. Bangladesh J. Pharmacol. 7, 217-221. doi:10.3329/bjp.v7i3.11727

Yan, P. S., Song, Y., Sakuno, E., Nakajima, H., Nakagawa, H., and Yabe, K. (2004). Cyclo(L-leucyl-L-prolyl) produced by Achromobacter xylosoxidans inhibits aflatoxin production by Aspergillus parasiticus. Appl. Environ. Microbiol. 70, 7466-7473. doi:10.1128/AEM.70.12.7466-7473.2004

Yan, W., Li, T., and Jiang, Z. (2010). Antiviral activity of Cordyceps guangdongensis against influenza virus infections in mice. Acta Edulis Fungi. 17, 64-66.

Yan, W., Li, T., Lao, J., Song, B., and Shen, Y. (2013). Anti-fatigue property of Cordyceps guangdongensis and the underlying mechanisms. Pharmaceut. Biol. 51, 614-620. doi:10.3109/13880209.2012.760103

Yan, W., Li, T., and Zhong, Z. (2014). Anti-inflammatory effect of a novel food Cordyceps guangdongensis on experimental rats with chronic bronchitis induced by tobacco smoking. Food Funct. 5, 2552-2557. doi:10.1039/c4fo00294f

Yan, W. J., Li, T. H., and Jiang, Z. D. (2011). Anti-fatigue and life-prolonging effects of Cordyceps guangdongensis. Food Res. Dev. 32, 164-166. doi:10.3109/ 13880209.2012 .760103

Yang, F. Q., Feng, K., Zhao, J., and Li, S. P. (2009). Analysis of sterols and fatty acids in natural and cultured Cordyceps by one-step derivatization followed with gas chromatography-mass spectrometry. J. Pharmaceut. Biomed. Anal. 49, 1172-1178. doi:10.1016/j.jpba.2009.02.025

Yang, J., Zhang, W., Shi, P., Chen, J., Han, X., and Wang, Y. (2005). Effects of exopolysaccharide fraction (EPSF) from a cultivated Cordyceps sinensis fungus on c-Myc, c-Fos, and VEGF expression in B16 melanoma-bearing mice. Pathol. Res. Pract. 201, 745-750. doi:10.1016/j.prp.2005.08.007

Yang, L. Y., Huang, W. J., Hsieh, H. G., and Lin, C. Y. (2003). H1-A extracted from Cordyceps sinensis suppresses the proliferation of human mesangial cells and promotes apoptosis, probably by inhibiting the tyrosine phosphorylation of Bcl-2 and Bcl-XL. J. Lab. Clin. Med. 141, 74-83. doi:10.1067/mlc.2003.6

Yang, M. L., Kuo, P. C., Hwang, T. L., and Wu, T. S. (2011). Anti-inflammatory principles from Cordyceps sinensis. J. Nat. Prod. 74, 1996-2000. doi:10.1021/np100902f

Yang, Q., Yin, Y., Yu, G., Jin, Y., Ye, X., Shrestha, A., et al. (2015a). A novel protein with anti-metastasis activity on $4 \mathrm{~T} 1$ carcinoma from medicinal fungus Cordyceps militaris. Int. J. Biol. Macromol. 80, 385-391. doi:10.1016/j.ijbiomac.2015.06.050

Yang, W. S., Nam, G. S., Kim, M. Y., and Cho, J. Y. (2017). Syk-mediated suppression of inflammatory responses by Cordyceps bassiana. Am. J. Chin. Med. 45, 1217-1232. doi:10.1142/S0192415X17500677

Yang, W. S., Ratan, Z. A., Kim, G., Lee, Y., Kim, M. Y., Kim, J. H., et al. (2015b). 4Isopropyl-2,6-bis(1-phenylethyl)aniline 1, an analogue of KTH-13 isolated from Cordyceps bassiana, inhibits the NF-kB-Mediated inflammatory response. Mediat. Inflamm., 143025 2015. doi:10.1155/2015/143025

Yoo, H. S., Shin, J. W., Cho, J. H., Son, C. G., Lee, Y. W., Park, S. Y., et al. (2004). Effects of Cordyceps militaris extract on angiogenesis and tumor growth. Acta Pharmacol. Sin. 25, 657-665.

Yoon, D. H., Han, C., Fang, Y., Gundeti, S., Han Lee, I. S., Song, W. O., Hwang, K. C., Kim, T. W., Sung, G. H., Park, H., et al. (2017). Inhibitory Activity of Cordyceps bassiana Extract on LPS-induced Inflammation in RAW 264.7 Cells by Suppressing NF-kB Activation. Nat. Prod. Sci. 23(3), 162-168. doi:10.20307/nps.2017.23.3.162

Yoon, J. Y., Kim, J. H., Baek, K. S., Kim, G. S., Lee, S. E., Lee, D. Y., et al. (2015). A direct protein kinase B-targeted anti-inflammatory activity of cordycepin from artificially cultured fruit body of Cordyceps militaris. Phcog. Mag. 11, 477-485. doi:10.4103/0973-1296.160454

Yoshida, J., Takamura, S., Yamaguchi, N., Ren, L. J., Chen, H., Koshimura, S., et al. (1989). Antitumor activity of an extract of Cordyceps sinensis (Berk.) Sacc. against murine tumor cell lines. Jpn. J. Exp. Med. 59, 157-161. 
Yoshikawa, N., Nakamura, K., Yamaguchi, Y., Kagota, S., Shinozuka, K., and Kunitomo, M. (2004). Antitumour activity of cordycepin in mice. Clin. Exp. Pharmacol. Physiol. 31 (Suppl. 2), S51-S53. doi:10.1111/j.1440-1681.2004. 04108.x

Yoshikawa, N., Nakamura, K., Yamaguchi, Y., Kagota, S., Shinozuka, K., and Kunitomo, M. (2007). Cordycepin and Cordyceps sinensis reduce the growth of human promyelocytic leukaemia cells through the Wnt signaling pathway. Clin. Exp. Pharmacol. Physiol. 34, S61-S63. doi:10.1111/j.14401681.2007.04781.x

Yoshikawa, N., Yamada, S., Takeuchi, C., Kagota, S., Shinozuka, K., Kunitomo, M., et al. (2008). Cordycepin (3'-deoxyadenosine) inhibits the growth of B16-BL6 mouse melanoma cells through the stimulation of adenosine A3 receptor followed by glycogen synthase kinase-3beta activation and cyclin D1 suppression. Naunyn-Schmiedeberg's Arch. Pharmacol. 377, 591-595. doi:10. 1007/s00210-007-0218-y

Yu, H. M., Wang, B. S., Huang, S. C., and Duh, P. D. (2006). Comparison of protective effects between cultured Cordyceps militaris and natural Cordyceps sinensis against oxidative damage. J. Agric. Food Chem. 54, 3132-3138. doi:10. 1021/jf053111w

Yu, L. X., Zhang, B. B., Ruan, Y. P., Yao, G. T., Xie, L. P., and Lu, K. D. (2004). Effects of different components of Cordyceps sinensis polysaccharide on immune function. J Zhejiang Coll Tradit Chin Med. 28, 49-50.

Yu, L., Zhao, J., Li, S. P., Fan, H., Hong, M., Wang, Y. T., et al. (2006). Quality evaluation of Cordyceps through simultaneous determination of eleven nucleosides and bases by RP-HPLC. J. Separ. Sci. 29, 953-958. doi:10.1002/ jssc. 200600007

Yu, L., Zhao, J., Zhu, Q., and Li, S. P. (2007). Macrophage biospecific extraction and high performance liquid chromatography for hypothesis of immunological active components in Cordyceps sinensis. J. Pharmaceut. Biomed. Anal. 44, 439-443. doi:10.1016/j.jpba.2007.01.003

Yu, R., Yin, Y., Yang, W., Ma, W., Yang, L., Chen, X., et al. (2009). Structural elucidation and biological activity of a novel polysaccharide by alkaline extraction from cultured Cordyceps militaris. Carbohydr. Polym. 75, 166-171. doi:10.1016/j.carbpol.2008.07.023

Yu, S. H., Dubey, N. K., Li, W. S., Liu, M. C., Chiang, H. S., Leu, S. J., et al. (2016). Cordyceps militaris treatment preserves renal function in type 2 diabetic nephropathy mice. PloS One. 11, e0166342. doi:10.1371/journal.pone.0166342

Zeng, H. B., Li, T. H., Song, B., Lin, Q. Y., and Huang, H. (2009). Study on antioxidant activity of Cordyceps guangdongensis. Nat. Prod. Res. Dev. 21, 201-204.

Zhan, Z. J., Fan, C. Q., Ding, J., and Yue, J. M. (2005). Novel diterpenoids with potent inhibitory activity against endothelium cell HMEC and cytotoxic activities from a well-known TCM plant Daphne genkwa. Bioorg. Med. Chem. 13, 645-655. doi:10.1016/j.bmc.2004.10.054

Zhang, G., Yin, Q., Han, T., Zhao, Y., Su, J., Li, M., et al. (2015). Purification and antioxidant effect of novel fungal polysaccharides from the stroma of Cordyceps kyushuensis. Ind. Crop. Prod. 69, 485-491. doi:10.1016/j.indcrop.2015.03.006

Zhang, J., Yu, Y., Zhang, Z., Ding, Y., Dai, X., and Li, Y. (2011). Effect of polysaccharide from cultured Cordyceps sinensis on immune function and anti-oxidation activity of mice exposed to 60Co. Int. Immunopharm. 11, 2251-2257. doi:10.1016/j.intimp.2011.09.019

Zhang, Q., Leung, P., Jianyong, W. U., and Zongding, H. U. (2005). Comparison of antitumor effect of extracts in cultivated Cordyceps sinensis fungus HK-1 and natural Cordyceps sinensis. Chin. Tradit. Herb. Drugs. 36, 1346-1349.

Zhang, Q., Olatunji, O. J., Chen, H., Tola, A. J., and Oluwaniyi, O. O. (2018). Evaluation of the anti-diabetic activity of polysaccharide from Cordyceps cicadae in experimental diabetic rats. Chem. Biodivers. 15, e1800219. doi:10. 1002/cbdv.201800219

Zhang, Q., Wu, J., Hu, Z., and Li, D. (2004). Induction of HL-60 apoptosis by ethyl acetate extract of Cordyceps sinensis fungal mycelium. Life Sci. 75, 2911-2919. doi:10.1016/j.lfs.2004.05.029

Zhang, W., Li, J., Qiu, S., Chen, J., and Zheng, Y. (2008). Effects of the exopolysaccharide fraction (EPSF) from a cultivated Cordyceps sinensis on immunocytes of H22 tumor bearing mice. Fitoterapia. 79, 168-173. doi:10. 1016/j.fitote.2007.09.001

Zhang, W., Yang, J., Chen, J., Hou, Y., and Han, X. (2005). Immunomodulatory and antitumour effects of an exopolysaccharide fraction from cultivated
Cordyceps sinensis (Chinese caterpillar fungus) on tumour-bearing mice. Biotechnol. Appl. Biochem. 42, 9-15. doi:10.1042/BA20040183

Zhang, Y., Liu, S., Liu, H., Liu, X., and Che, Y. (2009). Cycloaspeptides F and G, cyclic pentapeptides from a cordyceps-colonizing isolate of Isaria farinosa. J. Nat. Prod. 72, 1364-1367. doi:10.1021/np900205m

Zhang, Y., Wu, Y.-T., Zheng, W., Han, X.-X., Jiang, Y.-H., Hu, P.-L., et al. (2017). The antibacterial activity and antibacterial mechanism of a polysaccharide from Cordyceps cicadae. J. Funct. Foods. 38, 273-279. doi:10.1016/j.jff.2017. 09.047

Zhang, Z., Huang, W., Liao, S., Li, J., Lei, L., Lui, J., et al. (1995). Clinical and laboratory studies of JinShuiBao in scavenging oxygen free radicals in elderly senescent XuZheng patients. J. Admin. Trad. Chin. Med. 5, 14-18.

Zhao, C. S., Yin, W. T., Wang, J. Y., Zhang, Y., Yu, H., Cooper, R., et al. (2002). $\operatorname{CordyMax}^{\mathrm{TM}}$ Cs-4 improves glucose metabolism and increases insulin sensitivity in normal rats. J. Alternative Compl. Med. 8, 309-314. doi:10. 1089/10755530260127998

Zhao, X., Yu, X.-H., Zhang, G.-Y., Zhang, H.-Y., Liu, W.-W., Zhang, C.-K., et al. (2018). Aqueous extracts of Cordyceps kyushuensis kob induce apoptosis to exert anticancer activity. BioMed Res. Int. 2018, 8412098. doi:10.1155/2018/8412098

Zhou, X., Gong, Z., Su, Y., Lin, J., and Tang, K. (2009). Cordyceps fungi: natural products, pharmacological functions and developmental products. J. Pharm. Pharmacol. 61, 279-291. doi:10.1211/jpp/61.03.0002

Zhou, X., Luo, L., Dressel, W., Shadier, G., Krumbiegel, D., Schmidtke, P., et al. (2008). Cordycepin is an immunoregulatory active ingredient of Cordyceps sinensis. Am. J. Chin. Med. 36, 967-980. doi:10.1142/S0192415X08006387

Zhu, J. S., Halpern, G. M., and Jones, K. (1998). The scientific rediscovery of an ancient Chinese herbal medicine: Cordyceps sinensis Part I. J. Alternative Compl. Med. 4, 289-303. doi:10.1089/acm.1998.4.3-289

Zhu, L., Tang, Q., Zhou, S., Liu, Y., Zhang, Z., Gao, X., et al. (2014). Isolation and purification of a polysaccharide from the caterpillar medicinal mushroom Cordyceps militaris (ascomycetes) fruit bodies and its immunomodulation of RAW 264.7 macrophages. Int. J. Med. Mushrooms. 16, 247-257. doi:10.1615/ intjmedmushr.v16.i3.50

Zhu, R., Zheng, R., Deng, Y., Chen, Y., and Zhang, S. (2014). Ergosterol peroxide from Cordyceps cicadae ameliorates TGF- $\beta 1$-induced activation of kidney fibroblasts. Phytomedicine. 21, 372-378. doi:10.1016/j.phymed.2013. 08.022

Zhu, Z.-Y., Dong, F., Liu, X., Lv, Q., Yingyang, F., Chen, L., et al. (2016). Effects of extraction methods on the yield, chemical structure and anti-tumor activity of polysaccharides from Cordyceps gunnii mycelia. Carbohydr. Polym. 140, 461-471. doi:10.1016/j.carbpol.2015.12.053

Zhu, Z.-Y., Liu, X. C., Tang, Y. L., Dong, F. Y., Sun, H. Q., Chen, L., et al. (2016b). Effects of cultural medium on the formation and antitumor activity of polysaccharides by Cordyceps gunnii. J. Biosci. Bioeng. 122, 494-498. doi:10. 1016/j.jbiosc.2016.03.015

Zhu, Z.-Y., Liu, N., Si, C.-L., Liu, Y., Ding, L.-N., Jing, C., et al. (2012a). Structure and anti-tumor activity of a high-molecular-weight polysaccharide from cultured mycelium of Cordyceps gunnii. Carbohydr. Polym. 88, 1072-1076. doi:10.1016/j.carbpol.2012.01.068

Zhu, Z.-Y., Chen, J., Si, C. L., Liu, N., Lian, H. Y., Ding, L. N., et al. (2012b). Immunomodulatory effect of polysaccharides from submerged cultured Cordyceps gunnii. Pharm. Biol. 50, 1103-1110. doi:10.3109/13880209.2012. 658114

Zhu, Z.-Y., Pang, W., Li, Y.-Y., Ge, X.-R., Chen, L.-J., Liu, X.-C., et al. (2014). Effect of ultrasonic treatment on structure and antitumor activity of mycelial polysaccharides from Cordyceps gunnii. Carbohydr. Polym. 114, 12-20. doi:10.1016/j.carbpol.2014.07.068

Zhu, Z.-Y., Si, C.-L., Zhong, Y.-R., Zhu, C.-M., Zhou, J.-P., Liu, A.-J., et al. (2011). The purification and antioxidative activities in d-galactose-induced aging mice of a water-soluble polysaccharide from Cordyceps gunnii (Berk.) Berk. mycelium. J. Food Biochem. 35, 303-322. doi:10.1111/j.1745-4514.2010.00383.x

Zhu, Z. Y., Liu, Y., Si, C. L., Yuan, J., Lv, Q., Li, Y. Y., et al. (2013). Sulfated modification of the polysaccharide from Cordyceps_gunnii mycelia and its biological activities. Carbohydr. Polym. 92, 872-876. doi:10.1016/j.carbpol.2012.10.007

Zhu, Z. Y., Si, C. L., and Zhang, Y. M. (2009). Purification and antioxidative activities of a water-soluble polysaccharide isolated from Cordyceps gunnii (berk mycelium). Planta Med. 75, PC15. doi:10.1055/s-0029-1234474 
Conflict of Interest: The authors declare that the research was conducted in the absence of any commercial or financial relationships that could be construed as a potential conflict of interest.

Copyright (c) 2021 Das, Shin, Leyva-Gómez, Prado-Audelo, Cortes, Singh, Panda, Mishra, Nigam, Saklani, Chaturi, Martorell, Cruz-Martins, Sharma, Garg, Sharma and Patra. This is an open-access article distributed under the terms of the Creative Commons Attribution License (CC BY). The use, distribution or reproduction in other forums is permitted, provided the original author(s) and the copyright owner(s) are credited and that the original publication in this journal is cited, in accordance with accepted academic practice. No use, distribution or reproduction is permitted which does not comply with these terms. 